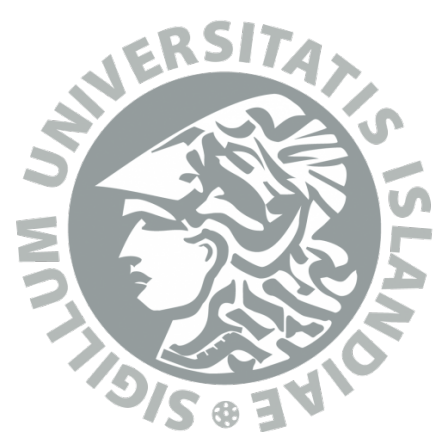

\title{
Nonperturbative Approach to Circuit Quantum Electrodynamics
}

Ólafur Jónasson

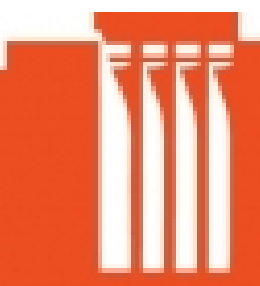

School of Engineering and

Natural Sciences

University of Iceland

2012 



\title{
NONPERTURBATIVE APPROACH TO CIRCUIT QUANTUM ELECTRODYNAMICS
}

\author{
Ólafur Jónasson
}

60 ECTS thesis submitted in partial fulfillment of a Magister Scientiarum degree in Physics

\author{
Advisor \\ Viðar Guð̆mundsson
}

\author{
M.Sc. committee \\ Viðar Guð̃mundsson \\ Andrei Manolescu
}

School of Engineering and Natural Sciences University of Iceland

Reykjavik, February 2012 
Nonperturbative Approach to Circuit Quantum Electrodynamics Nonperturbative Approach to C-QED

60 ECTS thesis submitted in partial fulfillment of a M.Sc. degree in Physics

Copyright @ 2012 Ólafur Jónasson

All rights reserved

School of Engineering and Natural Sciences

University of Iceland

Hjarðarhagi 2-6

107, Reykjavik, Reykjavik

Iceland

Telephone: 5254700

Bibliographic information:

Ólafur Jónasson, 2012, Nonperturbative Approach to Circuit Quantum Electrodynamics, M.Sc. thesis, School of Engineering and Natural Sciences, University of Iceland.

Printing: Háskólaprent, Fálkagata 2, 107 Reykjavík

Reykjavik, Iceland, February 2012 


\section{Abstract}

We investigate the coupling between a quantized electromagnetic field in a cavity resonator and a Coulomb interacting electronic system in a nanostructure in an external magnetic field. We use a nonperturbative approach with a stepwise introduction of complexity to the description of the system and a corresponding stepwise truncation of the ensuing many-body spaces. We are able to explicitly take into account effects caused by the geometry of the electronic system and the polarization of the electromagnetic field, including both the full interaction between electrons and photons and Coulomb interaction between electrons. Our numerical results demonstrate that the two-level system approximation and the Jaynes-Cummings model remain valid in the weak electron-photon coupling regime, while the quadratic vector potential in the diamagnetic part of the charge current leads to significant correction to the energy spectrum in the strong coupling regime. Investigation of numerical convergence shows that convergence with respect to the number of electron eigenstates is slow, requiring a large basis of many-electron eigenstates to be included in the model.

\section{Útdráttur}

Við rannsökum tengsl milli skammtaðs rafsegulsviðs í hermuholrúmi og Coulomb víxlverkandi rafeindakerfis í ytra segulsviði á nanóskala. Við notum aðferð sem byggist ekki á truflunarreikningi par sem Fock rými er stækkað skref fyrir skref við lýsingu á kerfinu og samsvarandi niðurskurður skref fyrir skref er gerður á margra-einda rúminu. Fullt tillit er tekið til lögunar rafeindakerfisins og skautunar rafsegulsviðsins. Líkanið inniheldur bæði fulla víxlverkun rafeinda og ljóseinda og Coulomb víxlverkunar milli rafeinda. Tölulegar niðurstöður sýna að nálgun með tvístiga kerfi og Jaynes-Cummings líkani gilda fyrir veik rafeinda-ljóseinda tengsl en annars stigs liðurinn fyrir vigurmætti rafsegulsviðsins hefur mikil áhrif fyrir sterk tengsli. Rannsóknir á tölulegri samleitni sýna að samleitni með tilliti til fjölda rafeinda eiginástanda er hæg og pví parf stóran grunn af margra rafeinda eiginástöndum í líkaninu. 



\section{Contents}

List of Figures $\quad$ ix

List of Tables $\quad$ XV

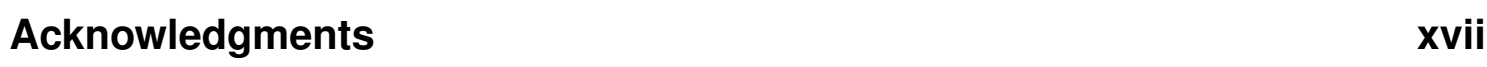

1. Introduction 1

2. Single electron system 3

2.1. The Hamiltonian of the central system . . . . . . . . . . . . . 3

2.2. Matrix elements . . . . . . . . . . . . . . . 4

2.3. Bijection ......................... 5

2.4. Diagonalization .................... 7

3. Many-electrons calculations 9

3.1. Formalism . . . . . . . . . . . . . . . . . . 9

3.2. Observables in second quantization . . . . . . . . . . . . . . . 11

3.3. Many electron Hamiltonian . . . . . . . . . . . . . . . . . . . . . . . 12

4. Inclusion of a quantized EM field 15

4.1. Quantized electromagnetic field in a cavity . . . . . . . . . . . . . . 15

4.2. The Hamiltonian . . . . . . . . . . . . . . . . . . . 16

4.3. The Jaynes-Cummings model . . . . . . . . . . . . . . . . . . . . . 19

4.4. Comparison of JC-model with exact model . . . . . . . . . . . . 20

4.4.1. Single electron and no magnetic field . . . . . . . . . . . . 20

4.4.2. Many electrons and non-zero magnetic field . . . . . . . . . . . . 21

5. Results 25

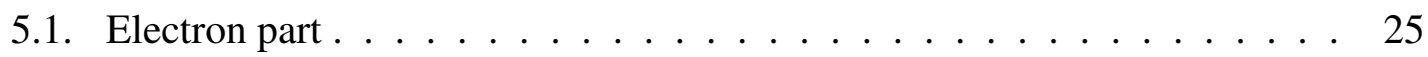

5.2. Full Model . . . . . . . . . . . . . . . . . . . . . . 28

5.2.1. Energy Spectra . . . . . . . . . . . . . . . . . . . 28

5.2.2. Charge Density . . . . . . . . . . . . . . . 36

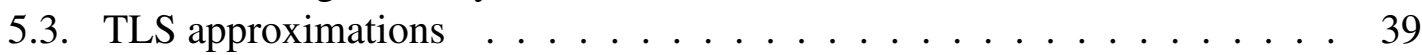

6. Convergence tests 47

6.1. One electron . . . . . . . . . . . . . . . . . . . . . 48 
6.2. More than one electron . . . . . . . . . . . . . . . . . . 49

7. Conclusion 55

A. Detailed calculations of matrix elements 57 A.1. Evaluating $I_{\mathrm{x}}^{\mathrm{nn}^{\prime}} \ldots \ldots \ldots \ldots \ldots \ldots \ldots \ldots \ldots$

A.2. Evaluating $I_{\mathbf{y}}^{\mathbf{m m}^{\prime}} \ldots \ldots \ldots \ldots \ldots \ldots \ldots \ldots \ldots$

A.3. Evaluating $G_{\mathbf{y}}^{\mathrm{mm}^{\prime}} \ldots \ldots \ldots \ldots \ldots \ldots \ldots$

A.4. Evaluating $g_{i j} \ldots \ldots \ldots \ldots \ldots \ldots \ldots$

B. Convergence of Coulomb matrix elements 63

$\begin{array}{ll}\text { Bibliography } & 67\end{array}$ 


\section{List of Figures}

2.1. Plot of $\ln \left(\left|\left(H_{0}\right)_{i j}\right|+1\right)$ for $m=6$ and $n=20$. The reason for the log plot is that the diagonal elements are much larger than the off diagonal ones. As we can see from the figure, the matrix consists of $\left(N_{y}+1\right) \times\left(N_{y}+1\right)=$ $7 \times 7$ blocks, each block being $N_{x} \times N_{x}=20 \times 20$. Within each of the blocks $m$ and $m^{\prime}$ are constant but $n$ and $n^{\prime}$ are varied. . . . . . . . . . .

5.1. Charge density (scaled by $-q$ ) of the lowest 6 Coulomb interacting one electron eigenstates. By comparison with equations (2.6)-(2.7) we can see that the six charge densities correspond roughly to the basis states $\phi_{1}(x) \varphi_{1}(y), \phi_{2}(x) \varphi_{1}(y), \phi_{3}(x) \varphi_{1}(y), \phi_{4}(x) \varphi_{1}(y), \phi_{1}(x) \varphi_{2}(y)$ and $\phi_{2}(x) \varphi_{2}(y)$. By roughly I mean that due to the non-zero magnetic field, $n$ and $m$ are not good quantum numbers. . . . . . . . . . . . . . 26

5.2. Charge density (scaled by $-q$ ) of the lowest 6 Coulomb interacting two electron eigenstates. . . . . . . . . . . . . . . 26

5.3. Charge density (scaled by $-q$ ) of the lowest 6 Coulomb interacting three electron eigenstates. . . . . . . . . . . . . . . . . . .

5.4. DGC strength between the 6 lowest Coulomb interacting eigenstates for both polarizations and 1, 2 and 3 electrons. In other words, it's a colormap plot of the matrix $|\mathcal{G}|$, whose matrix elements are $\left|\mathcal{G}_{\mu \nu}\right|$. From the plot we see that for $x$-polarization, the states most strongly coupled to the ground state are $\left.\left.\mid 2)_{1}, \mid 2\right)_{2}, \mid 2\right)_{3}$ and $\left.\mid 3\right)_{3}$. For $y$-polarization, the state $\left.\mid 5\right)$ is most strongly coupled for one, two and three electrons. . . . . . . . . .

5.5. Energy spectra for one electron and both $x$ (left) and $y$ (right) polarization. The lowest 64 states are plotted in both cases. For the $x$-polarization, the system is on resonance between the states $\mid 1)_{1}$ and $\left.\mid 2\right)_{1}$ with a DGC strength of $\left|\mathcal{G}_{12}\right|=0.290$ and $\hbar \omega_{p}=0.185 \mathrm{meV}$. For $y$-polarization, the system is on resonance between the states $\mid 1)_{1}$ and $\left.\mid 5\right)_{1}$ with $\left|\mathcal{G}_{15}\right|=0.701$ and $\hbar \omega_{p}=1.03 \mathrm{meV}$. The color coding is used later on to identify states when plotting charge densities in figures 5.12 and 5.13 . . . . . . . . . 29 


\section{LIST OF FIGURES}

5.6. Energy spectra for two electrons and both $x$ (left) and $y$ (right) polarization. The lowest 64 states are plotted in both cases. For the $x$-polarization, the system is on resonance between the states $\mid 1)_{2}$ and $\left.\mid 2\right)_{2}$ with a DGC strength of $\left|\mathcal{G}_{12}\right|=0.648$ and $\hbar \omega_{p}=0.516 \mathrm{meV}$. For $y$-polarization, the system is on resonance between the states $\mid 1)_{2}$ and $\left.\mid 5\right)_{2}$ with $\left|\mathcal{G}_{15}\right|=0.987$ and $\hbar \omega_{p}=1.025 \mathrm{meV} \ldots \ldots \ldots \ldots \ldots$

5.7. Energy spectra for three electrons and both $x$ (left) and $y$ (right) polarization. The lowest 64 states are plotted in both cases. For the $x$-polarization, the system is on resonance between the states $\mid 1)_{3}$ and $\left.\mid 3\right)_{3}$ with a DGC strength of $\left|\mathcal{G}_{12}\right|=0.587$ and $\hbar \omega_{p}=0.643 \mathrm{meV}$. For $y$-polarization, the system is on resonance between the states $\mid 1)_{3}$ and $\left.\mid 5\right)_{3}$ with a DGC strength of $\left|\mathcal{G}_{15}\right|=1.168$ and $\hbar \omega_{p}=1.020 \mathrm{meV}$. . . . . . . . . . .

5.8. Energy spectra for one electron and both $x$ (left) and $y$ (right) polarization. The lowest 64 states are plotted in both cases. The system is on resonance between the weakly coupled states $\mid 1)_{1}$ and $\left.\mid 3\right)_{1}$ with a DGC strength of $\left|\mathcal{G}_{13}\right| \sim 10^{-16}$ and $\hbar \omega_{p}=0.492$ for both polarizations. The spectrum is very chaotic for the $x$-polarization, while the one for the $y$-polarization is relatively smooth. . . . . . . . . . . . . . . .

5.9. Energy spectra for two electrons and both $x$ (left) and $y$ (right) polarization. The lowest 64 states are plotted in both cases. The system is on resonance between the weakly coupled states $\mid 1)_{2}$ and $\left.\mid 3\right)_{2}$ with a DGC strength of $\left|\mathcal{G}_{13}\right| \sim 10^{-13}$ and $\hbar \omega_{p}=0.648$ for both polarizations. Note how smooth the $x$-polarization spectrum is compared with the one electron case in Fig. 5.8. . . . . . . . . . . . . . . . . . . . .

5.10. Energy spectra for one electron and $x$-polarization (left) and two electrons and $y$-polarization (right). The $A^{2}$ term in the e-EM interaction Hamiltonian is both included (blue) and omitted (red). For the $x$-polarization, the system is on resonance between the states $\mid 1)_{1}$ and $\left.\mid 2\right)_{1}$ with a DGC strength of $\left|\mathcal{G}_{12}\right|=0.290$ and $\hbar \omega_{p}=0.185 \mathrm{meV}$. For the $y$-polarization, the system is on resonance between the states $\mid 1)_{2}$ and $\left.\mid 5\right)_{2}$ with a DGC strength of $\left|\mathcal{G}_{15}\right|=0.987$ and $\hbar \omega_{p}=1.025 \mathrm{meV}$. As can be seen from the figure, omitting the $A^{2}$ term does give accurate results for small $\mathcal{E}_{c}$, while for large $\mathcal{E}_{c}$ the energy spectrum takes a steep dive downwards. This dive also takes place in the two electron case (right panel), however it can't be seen in the chosen range of $\mathcal{E}_{c}$. There is no physical significance in these dives since the results are highly divergent in those areas as can be seen

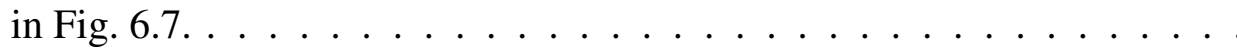


5.11. Energy spectra for two electrons and $x$-polarization (left) and one electron and $y$-polarization (right). The $A^{2}$ term in the e-EM interaction Hamiltonian is both included (blue) and omitted (red). For the $x$-polarization, the system is on resonance between the weakly coupled states $\mid 1)_{2}$ and $\mid 3)_{2}$ with a DGC strength of $\left|\mathcal{G}_{12}\right|=\sim 10^{-13}$ and $\hbar \omega_{p}=0.648 \mathrm{meV}$. For the $y$-polarization, the system is on resonance between the weakly coupled states $\mid 1)_{1}$ and $\left.\mid 3\right)_{1}$ with a DGC strength of $\left|\mathcal{G}_{13}\right| \sim 10^{-13}$ and $\hbar \omega_{p}=0.492 \mathrm{meV}$. Omitting the $A^{2}$ term for the case of $y$-polarization gives very bad results for the majority of the energy curves, even for very small $\mathcal{E}_{c}$. As for the $x$-polarization, we can see that for many of the energy curves, omitting the $A^{2}$ term gives satisfactory results. However, the discrepancy is large for some states, especially for the states $|3\rangle \otimes|0\rangle$ and $\mid 0) \otimes|1\rangle$, the states in which we are most interested in because those states will play a role in the TLS approximation later on. . . . . . . . . . . .

5.12. Electron charge densities (scaled by $-e$ ) for one electron and $x$-polarization. The system is on resonance between the states $\mid 1)_{1}$ and $\left.\mid 2\right)_{1}$ with a DGC strength of $\left|\mathcal{G}_{12}\right|=0.290$ and $\hbar \omega_{p}=0.185 \mathrm{meV}$. To identify the states, see the color coding on the left panel of Fig. 5.5 and the corresponding color marks on the $y$-axis above. Counting from top to bottom, the plotted states are $\mid 1)|0\rangle$ (blue), $\mid 2)|0\rangle$ (orange), $\mid 1)|1\rangle$ (gray), $\mid 2)|1\rangle$ (purple) and $\mid 3)|0\rangle$ (green). . . . . . . . . . . . . . . . . .

5.13. Electron charge densities (scaled by $-e$ ) for one electron and $y$-polarization. The system is on resonance between the states $\mid 1)_{1}$ and $\left.\mid 5\right)_{1}$ with a DGC strength of $\left|\mathcal{G}_{15}\right|=0.701$ and $\hbar \omega_{p}=1.03 \mathrm{meV}$. To identify the states, see the color coding on the right panel of Fig. 5.5 and the corresponding color marks on the $y$-axis above. Counting from top to bottom, the plotted states are $\mid 1)|0\rangle$ (blue), $\mid 4)|0\rangle$ (orange), $\mid 5)|0\rangle$ (gray), $\mid 1)|1\rangle$ (purple) and $\mid 6)|0\rangle$ (green). . . . . . . . . . . . . . .

5.14. Comparison of the many-body energy spectra versus the coupling strength $\mathcal{E}_{\mathrm{c}}$ for the case of one electron and $x$-polarization. The energy spectra's are obtained by the TLS model with (blue) and without (green) the $A^{2}$ term and the JC-model (red-dashed). The TLS model results are compared with the full numerical calculation results for the lowest active levels $\mid 1) \otimes|0\rangle$, $\mid 2) \otimes|0\rangle$, and $|1\rangle \otimes|1\rangle$ (purple). Other parameters are the same as for the $x$-polarization in Fig. 5.5 . . . . . . . . . . . . . 


\section{LIST OF FIGURES}

5.15. Comparison of the many-body energy spectra versus the coupling strength $\mathcal{E}_{\mathrm{c}}$ for the case of one electron and $y$-polarization. These energy states are obtained by TLS model including the $A^{2}$ term (blue), not including the $A^{2}$ term (green) and the JC-model (red-dashed). The TLS model results are compared with the full numerical calculation for the compared lowest active levels $\mid 1)|0\rangle, \mid 5)|0\rangle$ and $\mid 1)|1\rangle$ (purple) as well as inactive levels (gray). Other parameters are the same as for the $y$-polarization in Fig. 5.5. The inset shows the validity of the JC-model in the weak coupling limit. .

5.16. Comparison of the many-body energy spectra versus the coupling strength $\mathcal{E}_{\mathrm{c}}$ for the case of two electrons and $x$-polarization. These energy states are obtained by TLS model including the $A^{2}$ term (blue), not including the $A^{2}$ term (green) and the JC-model (red-dashed). The TLS model results are compared with the full numerical calculation for the compared lowest active levels $\left.\mid 1)_{2}|0\rangle, \mid 2\right)_{2}|0\rangle$ and $\left.\mid 1\right)_{2}|1\rangle$ (purple) as well as inactive levels (gray). Other parameters are the same as for the $x$-polarization in Fig. 5.6. The inset shows the validity of the JC-model in the weak coupling limit. .

5.17. Comparison of the many-body energy spectra versus the coupling strength $\mathcal{E}_{\mathrm{c}}$ for the case of two electrons and $y$-polarization. These energy states are obtained by TLS model including the $A^{2}$ term (blue), not including the $A^{2}$ term (green) and the JC-model (red-dashed). The TLS model results are compared with the full numerical calculation for the compared lowest active levels $\left.\mid 1)_{2}|0\rangle, \mid 5\right)_{2}|0\rangle$ and $\left.\mid 1\right)_{2}|1\rangle$ (purple) as well as inactive levels (gray). Other parameters are the same as for the $y$-polarization in Fig. 5.6. The inset shows the validity of the JC-model in the weak coupling limit.

5.18. Comparison of the many-body energy spectra versus the coupling strength $\mathcal{E}_{\mathrm{c}}$ for the case of two electrons and $y$-polarization. The system is on resonance between the weakly coupled states $\mid 1)_{2}$ and $\left.\mid 2\right)_{2}$ with a DGC strength of $\left|\mathcal{G}_{12}\right|=0.0772, \hbar \omega_{p}=0.516 \mathrm{meV}$ and $\lambda=0.593$. These energy states are obtained by TLS model including the $A^{2}$ term (blue), not including the $A^{2}$ term (green) and the JC approximation without magnetic field (red-dashed). The TLS model results are compared with the full numerical calculation for the compared lowest active levels $\left.\mid 1)_{2}|0\rangle, \mid 2\right)_{2}|0\rangle$ and $\mid 1)_{2}|1\rangle$ (purple) as well as inactive levels (gray). . . . . . . . . .

6.1. Convergence calculations with respect to $N_{\text {mest }}$ for $x$-polarization (a) and $y$-polarization (b). For this run we have $a=100, b=150, c=200$ and $d=250$ (see equations 6.1 and 6.2 for definition). The maximum number of photons is kept constant at $N_{\mathrm{EM}}=20 \ldots \ldots \ldots$. . . . . 
6.2. Convergence calculations with respect to $N_{\mathrm{EM}}$ for $x$-polarization (a) and $y$-polarization (b). For this run we have $a=10, b=15, c=20$ and $d=$ 25 (see equations 6.1 and 6.2 for definition). The electron state number is kept constant at $N_{\text {mesT }}=200$. We can see that for $N_{E M}=20$, the results are acceptable for the whole range of $\mathcal{E}_{c}$ considered. . . . . . . . . .

6.3. Convergence calculations for two electrons with respect to $N_{\text {mesT }}$ for $x$ polarization (a) and $y$-polarization (b). For this run we have $a=100$, $b=150, c=200$ and $d=250$ (see equations 6.1 and 6.2 for definition). Other accuracy parameters are $N_{\text {ses }}=50$ and $N_{\mathrm{EM}}=20 . \ldots 50$

6.4. Convergence calculations for two electrons with respect to $N_{\text {ses }}$ (a) and grid size (b). In both cases, $y$-polarization is used and we have resonance between the two electron states $\mid 1$ ) and $\mid 5)$ giving $\hbar \omega_{p}=1.025 \mathrm{meV}$. The corresponding results for $x$-polarization are almost identical and will be omitted. . . . . . . . . . . . . . . . . . .

6.5. Convergence calculations for three electrons with respect to $N_{\text {mesT }}$ for $x$ polarization (b) and $y$-polarization (b). For this run we have $a=100$, $b=150, c=200$ and $d=250$ (see equations 6.1 and 6.2 for definition). Other accuracy parameters are $N_{\text {ses }}=30$ and $N_{\mathrm{EM}}=20 \ldots \ldots$. . .

6.6. Convergence calculations for two electrons with respect to $N_{\text {mest }}$ for $x$ polarization (b) and $y$-polarization (b). The system is off resonance with $\hbar \omega_{p}=0.4 \mathrm{meV}$ for both polarizations. For this run we have $a=100$, $b=150, c=200$ and $d=250$ (see equations 6.1 and 6.2 for definition). Other accuracy parameters are $N_{\mathrm{ses}}=50$ and $N_{\mathrm{EM}}=20 . \ldots \ldots$

6.7. Convergence calculations with respect to $N_{\text {mest }}$ for $x$-polarization, ignoring the $A^{2}$ term in the e-EM interaction Hamiltonian. The system is on resonance between the two electron states $\mid 1)_{2}$ and $\left.\mid 2\right)_{2}$ giving $\hbar \omega_{p}=$ 0.516 . For this run we have $a=100, b=150, c=200$ and $d=250$ (see equations 6.1 and 6.2 for definition). The results start to blow up at around $\mathcal{E}_{c} \simeq 0.4$. At around $\mathcal{E}_{c} \simeq 0.7$, the error is a 1000 times larger than the calculated value of the energy. . . . . . . . . . . . 53 



\section{List of Tables}

3.1. $N_{\text {mes }}$ for some values of $N_{e}$ and $N_{\text {ses }} \ldots \ldots \ldots \ldots$

5.1. Energies of the 6 lowest states $\mid \mu$ ) for one, two and three electrons. The energy is in units of meV. . . . . . . . . . . . . . 28 



\section{Acknowledgments}

First and foremost, I would like to thank my thesis supervisor, teacher and co-author, prof. Viðar Guðmundsson. His teaching and positive attitude during my undergraduate years was an inspiration and no doubt played a major role in my choice of master program. I would also like to thank him for his guidance during my time working for him as an research assistant and for his superb master program supervision.

I would also like thank Chi-Shung Tang at the National United University in Taiwan for his help with article writing and proof reading.

My work on this thesis was funded by the Icelandic Research Fund and the Research Fund of the University of Iceland. 



\section{Introduction}

In the last decade there has been increasing interest in systems capable of generating quantized fields containing a preset number of photons. Manipulation of the state of scalable light-matter coupled quantum systems is one of the key issues for their implementation for optomechanical systems [1, 2] or quantum information processing devices [3, 4]. However, searching for a clear evidence of light-matter coupling nonlinearity is still a challenge. To this end, one has to reach a strong light-matter coupling regime for optically driven systems in high quality micro-cavities [5, 6], and demonstrate its single-photon characteristics $[7,8]$. Flexible experimental design of circuit quantum electrodynamics offers a great potential for practical device applications to explore strong light-matter coupling at microwave frequencies [9-13].

Recently, utilization of the giant dipole moments of intersubband transitions in quantum wells $[14,15]$ has enabled researchers to reach the ultrastrong light-matter coupling regime [16-18]. In this regime, simple models such as the Jaynes-Cummings (JC) model are not applicable and the coupling mechanism has to be explored beyond the JC-model [19-21]. Despite the above mentioned experiments, a study of the coupling between electrons and cavity photons with a specified nanostructure geometry in a perpendicular magnetic field is still lacking.

In this thesis, we investigate the interplay of the dynamics of correlated electrons in a nanostructure to the quantum field of a rectangular cavity resonator subject to an external magnetic field. By performing numerical computations we demonstrate how the electronphoton coupling influences an electronic system embedded in a quantized photon field. We use a nonperturbative approach to a many-body model, including the full interaction between electrons and photons. A two level system (TLS) approximation and the JCmodel will be examined in both the weak and the strong coupling regimes as well as the effects of the diamagnetic part of the charge current in the electron-photon interaction term, which the JC-model lacks. A large part of the material and results presented in this thesis was published in [22]. However, in this thesis we take a more in-depth view and take the comparison of the different models further.

This thesis is split into 7 chapters, the first of which is this introduction. In chapter 2 I introduce the details of the nanostructure and its single electron Hamiltonian. The most important result in that chapter will be the single electron eigenfunctions and energies which will be used extensively later on as a basis for the many-body calculations. In 


\section{Introduction}

chapter 3 I introduce the second quantization formalism which is necessary to describe exactly a many particle system which includes the mutual Coulomb interaction between the electrons. In chapter 4 I outline the theory needed to explain the electron-photon coupling between electrons in the nanostructure with the photon field of a micro-cavity. The electron-photon coupling will be treated both exactly as well with the much simpler TLSand JC-models. Results and analysis of the numerical results are presented in chapter 5 and the issue of numerical convergence is addressed in chapter 6. Finally, concluding remarks are presented in chapter 7.

Throughout this thesis, MKL units will be used exclusively. I will differentiate operators in Hilbert and Fock space with the use of a calligraphic font for Fock space operators. As an example, $A$ is an operator in Hilbert space, while $\mathcal{A}$ is its Fock space equivalent. The only exception to this is the density operator $\rho$, which is always a Fock space operator. Two and three dimensional vectors are denoted by a boldfaced character such as r. Complex conjugate of a complex number or function is denoted by a superscript asterisk. For example the complex conjugate of a complex valued function $\psi(\mathbf{r})$ is written as $\psi^{*}(\mathbf{r})$. 


\section{Single electron system}

\subsection{The Hamiltonian of the central system}

The system under investigation is a two-dimensional electronic nanostructure exposed to a quantized electromagnetic field of a cavity resonator and a static (classical) external magnetic field at a low temperature. The electronic nanostructure is assumed to be fabricated by split-gate configuration in the y-direction, forming a parabolic confinement with the characteristic frequency $\Omega_{0}$ on top of a semiconductor heterostructure. The ends of the nanostructure in the $\mathrm{x}$-direction at $x= \pm L_{x} / 2$ are etched, forming a hard-wall confinement of length $L_{x}$. Thereby, a closed electronic narrow constriction is created in the $2 \mathrm{D}$ electron gas. The external classical magnetic field is given by $\mathbf{B}=B \hat{\mathbf{z}}$ with a vector potential A. Hence, the Hamiltonian of the nano-structure can be expressed in first quantization as

$$
H_{0}=\frac{1}{2 m}(\mathbf{p}+q \mathbf{A})^{2}+\frac{1}{2} m \Omega_{0}^{2} y^{2},
$$

where $m$ is the effective mass of the electron, $-q$ its charge and $\mathbf{p}$ the canonical momentum operator. As can seen from the Hamiltonian above, the spin degree of freedom is neglected. This means we are technically not considering electrons, rather spin-less fermions with identical charge to an electron. However, throughout this thesis I will refer to the considered particles as electrons. Although neglecting spin can be somewhat justified due to the low electron spin g-factor in GaAs, the spin can still cause energy degeneracy and allow symmetric electron states (as long as the spin part is anti-symmetric). However, our numerical procedure of Hilbert/Fock space truncations is still work in progress and we believe including spin at this point would distract us from the main objective of this thesis.

To find the eigenstates $\left(\left|\psi_{i}\right\rangle\right)$ of $H_{0}$ and their corresponding energies $\left(E_{i}\right)$ we need to solve the time-independent Schrödinger equation

$$
H_{0}\left|\psi_{i}\right\rangle=E_{i}\left|\psi_{i}\right\rangle
$$

subject to the boundary conditions

$$
\begin{gathered}
\psi_{i}\left( \pm L_{x} / 2, y\right)=0 \\
\psi_{i}(x, \pm \infty)=0 .
\end{gathered}
$$




\section{Single electron system}

Choosing the Landau gauge so $\mathbf{A}=(-B y, 0,0)$ and expanding the first term on the r.h.s. in (2.1), we get

$$
H_{0}=\frac{1}{2 m} p_{x}^{2}+\frac{1}{2 m} p_{y}^{2}+\frac{1}{2} m \Omega_{w}^{2} y^{2}+i \omega_{c} y p_{x},
$$

where $\omega_{c}=\frac{q B}{m}$ and $\Omega_{w}=\sqrt{\omega_{c}^{2}+\Omega_{0}^{2}}$. The above Hamiltonian is an infinite square well in the $x$-direction, a harmonic oscillator in the $y$-direction with an additional mixing term $i \omega_{c} y p_{x}$. This term couples the $x$ - and $y$-directions, which means we can not use separation of variables to solve Eq. (2.2) for the given finite quantum wire and have to resort to numerical techniques.

It it useful to be able to put a potential well or hill anywhere we want on the quantum wire. With a linear combination of gaussian potentials it is possible to make all kind of structures. The most general $2 \mathrm{D}$ gaussian potential is of the form

$$
V_{G}=A_{G} \exp \left(-\beta_{x}^{2}\left(x-x_{o}\right)^{2}-\beta_{y}^{2}\left(y-y_{0}\right)^{2}\right),
$$

where $\mathbf{r}_{0}=\left(x_{0}, y_{0}\right)$ is the center of the potential, $\beta_{x}$ and $\beta_{y}$ control the range of the potential and $A_{G}$ is some constant with the unit of energy.

\subsection{Matrix elements}

Solutions to (2.2) are not separable in $x$ and $y$, but we can still solve it using a complete orthonormal basis. As a basis I choose $\{|n, m\rangle\} \equiv\left\{\left|\phi_{n}\right\rangle \otimes\left|\varphi_{m}\right\rangle\right\}$ where $\left|\phi_{n}\right\rangle$ are eigenfunctions to the infinite square well and $\left|\varphi_{m}\right\rangle$ to the harmonic oscillator. In coordinate representation

$$
\left\langle x \mid \phi_{n}\right\rangle=\left\{\begin{array}{lll}
\sqrt{\frac{2}{L_{x}}} \cos \left(\frac{n \pi}{L_{x}} x\right) & \text { if } & n=1,3,5, \ldots \\
\sqrt{\frac{2}{L_{x}}} \sin \left(\frac{n \pi}{L_{x}} x\right) & \text { if } & n=2,4,6, \ldots
\end{array}\right.
$$

and

$$
\left\langle y \mid \varphi_{m}\right\rangle=\frac{e^{-\frac{y^{2}}{2 a_{w}^{2}}}}{\sqrt{2^{m} \sqrt{\pi} m ! a_{w}}} H_{m}\left(y / a_{w}\right), \quad m=0,1,2, \ldots
$$

where $a_{w}=\sqrt{\frac{\hbar}{m \Omega_{w}}}$ is the characteristic length of the system and $H_{m}$ are Hermite polynomials.

We can now calculate the matrix elements $\left\langle n, m\left|H_{0}\right| n^{\prime}, m^{\prime}\right\rangle$;

$$
\begin{aligned}
\left\langle n, m\left|H_{0}\right| n^{\prime}, m^{\prime}\right\rangle & =\delta_{n, n^{\prime}} \delta_{m, m^{\prime}}\left\{\frac{n^{2} \pi^{2} \hbar^{2}}{2 m L_{x}^{2}}+\hbar \Omega_{c}(m+1 / 2)\right\}+i \omega_{c} \hbar \frac{a_{w}}{L_{x}} I_{x}^{n n^{\prime}} I_{y}^{m m^{\prime}} \\
& =\delta_{n, n^{\prime}} \delta_{m, m^{\prime}} \hbar \Omega_{c}\left\{\frac{a_{w}^{2}}{L_{x}^{2}} \frac{n^{2} \pi^{2}}{2}+m+1 / 2\right\}+i \omega_{c} \hbar \frac{a_{w}}{L_{x}} I_{x}^{n n^{\prime}} I_{y}^{m m^{\prime}},
\end{aligned}
$$


where

$$
I_{x}^{n n^{\prime}} \equiv\left\langle\phi_{n}\left|L_{x} \partial_{x}\right| \phi_{n^{\prime}}\right\rangle= \begin{cases}0, & \text { if }\left(n+n^{\prime}+1\right) \text { is odd } \\ \frac{-4 n n^{\prime}}{n^{2}-n^{\prime 2}}(-1)^{\left(n+n^{\prime}+1\right) / 2}, & \text { otherwise }\end{cases}
$$

and

$$
I_{y}^{m m^{\prime}} \equiv\left\langle\phi_{m}\left|y / a_{w}\right| \phi_{m^{\prime}}\right\rangle=\sqrt{\frac{m^{\prime}+1}{2}} \delta_{m, m^{\prime}+1}+\sqrt{\frac{m^{\prime}}{2}} \delta_{m, m^{\prime}-1} .
$$

For a gaussian potential of the form (2.5), the matrix element is

$$
V_{G}\left\langle\phi_{n}\left|e^{-\beta_{x}^{2}\left(x-x_{0}\right)^{2}}\right| \phi_{n^{\prime}}\right\rangle\left\langle\varphi_{m}\left|e^{-\beta_{y}^{2}\left(y-y_{0}\right)^{2}}\right| \varphi_{m^{\prime}}\right\rangle=V_{G} G_{x}^{n n^{\prime}} G_{y}^{m m^{\prime}} .
$$

Analytical solution of the $x$ integral in (2.11) requires the use of the scaled complex valued error function which is not implemented in Fortran. No stable third party implementation of it was found (excluding the NAG numerical library which is commercial) so it will be evaluated numerically using Gauss quadrature. The $y$ integral can be done analytically and the result is

$$
\begin{gathered}
G_{y}^{m m^{\prime}}=\frac{e^{-\frac{\alpha_{y}^{2} \xi_{0}^{2}}{\alpha_{y}^{2}+1}}\left(\frac{2 \alpha_{y}^{2} \xi_{0}}{\alpha_{y}^{2}+1}\right)^{m+m^{\prime}}}{\sqrt{2^{m+m^{\prime}} \pi m ! m^{\prime} !}} \sum_{k=0}^{m} \sum_{\ell=0}^{m^{\prime}} Q_{k \ell}\left(\begin{array}{c}
m \\
k
\end{array}\right)\left(\begin{array}{c}
m^{\prime} \\
\ell
\end{array}\right) \frac{\left(\alpha_{y}^{2}+1\right)^{k+\ell-1 / 2}}{\left(\alpha_{y}^{2} \xi_{0}\right)^{k+\ell}} \\
\times\left(\frac{-\alpha_{y}^{2}}{\alpha_{y}^{2}+1}\right)^{(\ell+k) / 2} \Gamma\left(\frac{k+\ell+1}{2}\right){ }_{2} F_{1}\left(-k,-\ell ; \frac{1-k-\ell}{2} ; \frac{\alpha_{y}^{2}+1}{2 \alpha_{y}^{2}}\right),
\end{gathered}
$$

where

$$
\alpha_{y}=\beta_{y} a_{w}, \quad \xi_{0}=\frac{y_{0}}{a_{w}} \neq 0, \quad \alpha_{y} \neq 0, \quad Q_{k \ell}=\left\{\begin{array}{l}
0, \text { if } k+\ell \text { is odd } \\
1, \text { otherwise }
\end{array}\right.
$$

where ${ }_{2} F_{1}$ is the hyper-geometric function. If $\alpha_{y}=0$ then $G_{y}^{m m^{\prime}}=\delta_{m m^{\prime}}$ and if $\xi_{0}=0$ then

$$
\begin{aligned}
G_{y}^{m m^{\prime}}\left(\xi_{0}=0\right) & =Q_{m m^{\prime}} \frac{2^{m+m^{\prime}}}{\sqrt{2^{m+m^{\prime}} \pi m ! m^{\prime} !}}\left(\alpha_{y}^{2}+1\right)^{-\left(m+m^{\prime}+1\right) / 2}\left(-\alpha_{y}^{2}\right)^{\left(m+m^{\prime}\right) / 2} \\
& \times \Gamma\left(\frac{m+m^{\prime}+1}{2}\right){ }_{2} F_{1}\left(-m,-m^{\prime} ; \frac{1-m-m^{\prime}}{2} ; \frac{\alpha_{y}^{2}+1}{2 \alpha_{y}^{2}}\right) .
\end{aligned}
$$

For detailed calculations of (2.9), (2.10), (2.12) and (2.14) see appendix A.

\subsection{Bijection}

At the moment we need two parameters (quantum numbers) to uniquely determine a function in the basis $(n$ and $m$ ). We want to be able to label the functions with one parameter 


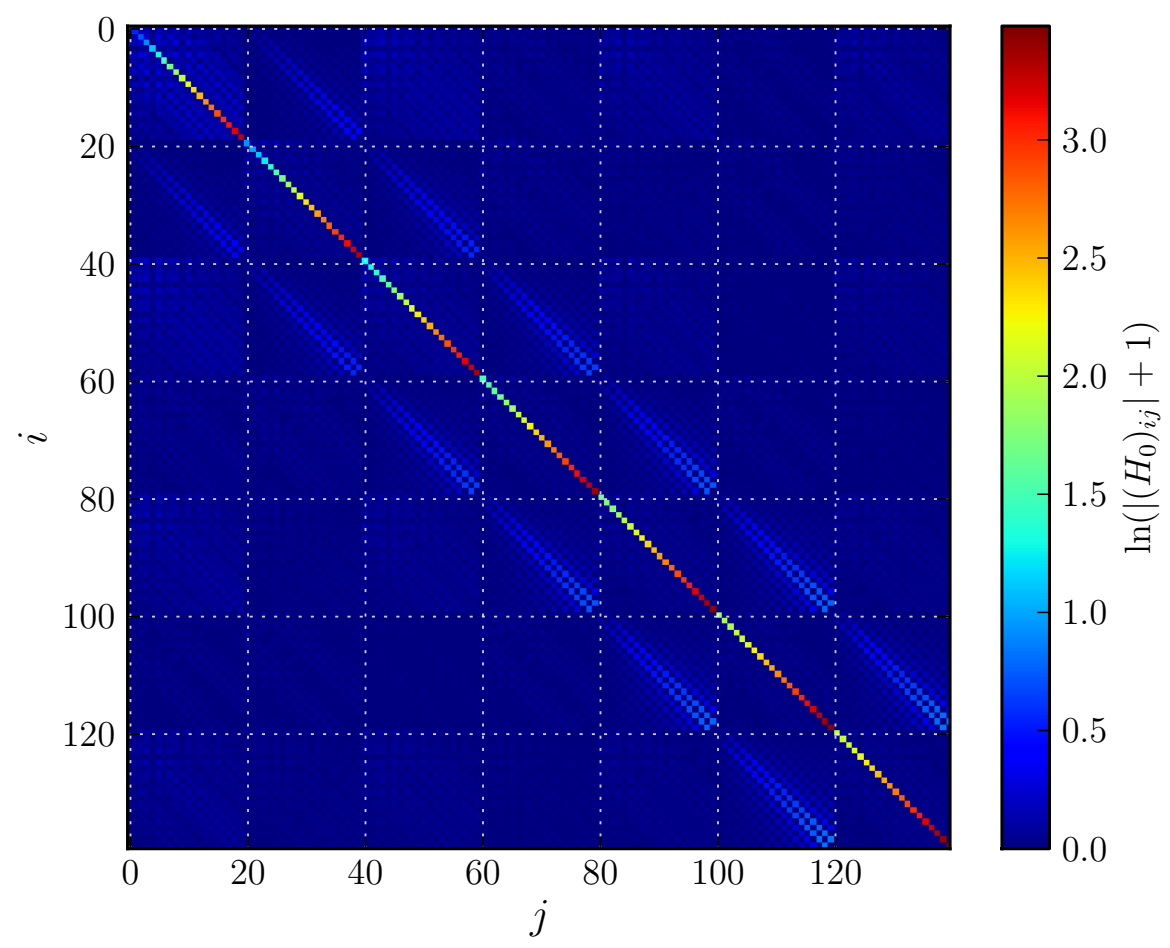

Figure 2.1: Plot of $\ln \left(\left|\left(H_{0}\right)_{i j}\right|+1\right)$ for $m=6$ and $n=20$. The reason for the log plot is that the diagonal elements are much larger than the off diagonal ones. As we can see from the figure, the matrix consists of $\left(N_{y}+1\right) \times\left(N_{y}+1\right)=7 \times 7$ blocks, each block being $N_{x} \times N_{x}=20 \times 20$. Within each of the blocks $m$ and $m^{\prime}$ are constant but $n$ and $n^{\prime}$ are varied.

only. We therefore need a bijection $\Gamma:(n, m) \rightarrow i$. In order to choose this bijection we need to decide the range of values $n$ and $m$ can take (truncate the basis). Let's denote the maximum value $n$ and $m$ can take as $N_{x}$ and $N_{y}$ which sets the total number of functions as $N_{x y}=\left(N_{y}+1\right) N_{x}$. A simple choice of $\Gamma$ is then

$$
i=\Gamma(n, m)=n+m N_{x},
$$

where $n \in\left[1, N_{x}\right]$ and $m \in\left[0, N_{y}\right]$. With this choice of bijection the matrix of $H_{0}$ will consist of $\left(N_{y}+1\right)^{2}$ blocks, one for each pair $\left(m, m^{\prime}\right)$ and each block will have $N_{x}^{2}$ elements or $N_{x}^{2}\left(N_{y}+1\right)^{2}=N_{x y}^{2}$ total elements (see Fig. 2.1). To calculate $n_{i}$ and $m_{i}$ from $i$ I use

$$
\begin{aligned}
n_{i} & =\left[(i-1) \bmod N_{x}\right]+1 \\
m_{i} & =\text { floor }\left(\frac{i-1}{N_{x}}\right)
\end{aligned}
$$

For example if we have $i=10$ and $N_{x}=7$ we have $n_{i}=[9 \bmod 7]+1=2+1=3$ and $m_{i}=$ floor $\left(\frac{9}{7}\right)=1$. 


\subsection{Diagonalization}

After calculating all the matrix elements of $H_{0}$ we can find it's eigenvalues $E_{i}$ and the corresponding eigenvectors $\left|\psi_{i}\right\rangle$. The eigenvalues are numbered in ascending order (starting with one). For the eigenvectors we have

$$
\left|\psi_{i}\right\rangle=\sum_{j=1}^{N_{x y}} U_{i j}\left(\left|\phi_{n_{j}}\right\rangle \otimes\left|\varphi_{m_{j}}\right\rangle\right)=\left(\begin{array}{c}
c_{1}^{(i)} \\
c_{2}^{(i)} \\
\vdots \\
c_{N_{x y}}^{(i)}
\end{array}\right), \quad H_{0}\left|\psi_{i}\right\rangle=E_{i}\left|\psi_{i}\right\rangle .
$$

Where $U$ is some unitary transformation that is calculated in the diagonalizaton process. The eigenvalues $E_{i}$ correspond to allowed energies of the system and $\left|\psi_{i}\right\rangle$ are the corresponding eigenvectors. The wave function of the state with energy $E_{i}$ can be calculated using

$$
\psi_{i}(\mathbf{r})=\left\langle\mathbf{r} \mid \psi_{i}\right\rangle=\sum_{j=1}^{N_{x y}} U_{i j} \phi_{n_{j}}(x) \varphi_{m_{j}}(y) .
$$

The only approximations we have made is the finite value of $N_{x}$ and $N_{y}$. The results become exact in the limit $N_{x}, N_{y} \rightarrow \infty$. Typical used values are $N_{x}=120$ and $N_{y}=30$. The reason for the higher value of $N_{x}$ is the particular values for the length of the wire and its $y$-confinement. Also, the functions in the $x$-direction are sines and cosines, which are numerically much easier to work with than high order Hermite polynomials. 



\section{Many-electrons calculations}

\subsection{Formalism}

To describe quantum states with a variable amount of particles I use the second quantization formulation of quantum mechanics. I specify a state in Fock space using greek indices such as $|\mu\rangle$ and a state in Hilbert space using latin indices such as $\left|\psi_{i}\right\rangle$, where $\mu, i \in \mathbb{N}_{+}$. If the $\left|\psi_{i}\right\rangle$ form a complete and orthonormal basis of the Hilbert space we are working in, then in the occupation number basis, a state in Fock space is defined as (see Ref. [23, 4-24])

$$
|\mu\rangle=\left|n_{1}^{\mu}, n_{2}^{\mu}, n_{3}^{\mu}, \cdots, n_{\infty}^{\mu}\right\rangle,
$$

which means that $n_{1}^{\mu}$ particles are in state $\left|\psi_{1}\right\rangle, n_{2}^{\mu}$ in state $\left|\psi_{2}\right\rangle$ etc. For fermions we have $n_{i}^{\mu}=0$ or $n_{i}^{\mu}=1$. For example

$$
|0,1,1,0,1,0,0, \ldots\rangle=\left|\psi_{2}\right\rangle \otimes\left|\psi_{3}\right\rangle \otimes\left|\psi_{5}\right\rangle
$$

We have

$$
\sum_{i=1}^{\infty} n_{i}^{\mu}=N_{\mu}
$$

where $N_{\mu}$ is the number of particles in state $|\mu\rangle$.

When doing calculations, the Fock space needs to be truncated by putting $\infty \rightarrow N_{\text {ses }}$ in (3.1), where $N_{\text {ses }}$ is a finite positive integer. This means we are using a finite number of single electron states to construct the Fock space. The corresponding number of many electron states (denoted $\left.N_{\text {mes }}\right)$ is $\left(\begin{array}{c}N_{\text {ses }} \\ N_{e}\end{array}\right)$ where $N_{e}$ is the number of electrons. This rapid growth of $N_{\text {mes }}$ with increasing $N_{e}$ or $N_{\text {ses }}$ greatly limits the number of electrons we can do calculations with because the matrix of the many electron Hamiltonian has the dimension $N_{\text {mes }} \times N_{\text {mes }}$. A reasonable value at the moment for $N_{\text {mes }}$ is about 5000 . An example of the rapid growth of $N_{\text {mes }}$ can bee seen in table 3.1.

We have defined the quantum states in second quantization formalism and now we define the operators that act on them. The fermionic creation $\left(d_{k}^{\dagger}\right)$ and destruction $\left(d_{k}\right)$ operators 
Table 3.1: $N_{\text {mes }}$ for some values of $N_{e}$ and $N_{\text {ses }}$

\begin{tabular}{c|cccccc}
$N_{e} \backslash N_{\text {ses }}$ & 8 & 16 & 24 & 32 & 40 & 48 \\
\hline 2 & 28 & 120 & 276 & 496 & 780 & 1128 \\
3 & 56 & 560 & 2024 & 4960 & 9880 & 17296 \\
4 & 70 & 1820 & 10626 & 35960 & 91390 & 194580 \\
\hline
\end{tabular}

are defined by their action on a state in Fock space.

$$
\begin{aligned}
d_{k}^{\dagger} d_{k}\left|\cdots n_{k} \cdots\right\rangle & =n_{k}\left|\cdots n_{k} \cdots\right\rangle \\
d_{k}\left|\cdots n_{k} \cdots\right\rangle & = \begin{cases}(-1)^{\gamma_{k}}|\cdots 0 \cdots\rangle, & \text { if } n_{k}=1 \\
0, & \text { if } n_{k}=0\end{cases} \\
d_{k}^{\dagger}\left|\cdots n_{k} \cdots\right\rangle & =\left\{\begin{array}{ll}
0, & \text { if } n_{k}=1 \\
(-1)^{\gamma_{k}}|\cdots 1 \cdots\rangle, & \text { if } n_{k}=0
\end{array},\right.
\end{aligned}
$$

where

$$
\gamma_{k}=\sum_{i=1}^{k-1} n_{i}
$$

It is straightforward to show that these operators satisfy the fermionic anti-commutation relations

$$
\left\{d_{i}, d_{j}\right\}=\left\{d_{i}^{\dagger}, d_{j}^{\dagger}\right\}=0,\left\{d_{i}, d_{j}^{\dagger}\right\}=\delta_{i j}
$$

A one-particle operator in second quantization (denoted by $\mathcal{A}$ ) can be represented using its first quantization variant (denoted by $A$ ) using

$$
\mathcal{A} \equiv \int \mathrm{d} \mathbf{r} \psi^{\dagger} A \psi=\sum_{i, j}\left\langle\psi_{i}|A| \psi_{j}\right\rangle d_{i}^{\dagger} d_{j},
$$

where

$$
\psi \equiv \sum_{i} \psi_{i}(\mathbf{r}) d_{i}, \psi^{\dagger} \equiv \sum_{i} \psi_{i}^{*}(\mathbf{r}) d_{i}^{\dagger}
$$

are fermionic field operators, with $d_{i}$ the annihilation- and $d_{i}^{\dagger}$ the creation operator for an electron in the single-electron state $|i\rangle$ corresponding to the eigenfunction $\psi_{i}(\mathbf{r})$. The $|i\rangle$ need not be eigenfunctions of the single electron Hamiltonian. It is sufficient that they form an orthonormal basis with the correct boundary conditions. However, this choice simplifies calculations and is therefore an obvious one.

Two-particle Fock space operators are defined as

$$
\mathcal{V} \equiv \frac{1}{2} \sum_{i j r s}\langle i j|V| r s\rangle d_{i}^{\dagger} d_{j}^{\dagger} d_{s} d_{r} .
$$


The matrix elements of a two-particle operator are thus expanded in the basis of the SESs involving the integration with respect to the observing location $\mathbf{r}$

$$
\langle i j|V| r s\rangle=\int d \mathbf{r} \psi_{i}^{*}(\mathbf{r}) \mathcal{I}_{j r}(\mathbf{r}) \psi_{s}(\mathbf{r})
$$

and the integration with respect to the source location $\mathbf{r}^{\prime}$

$$
\mathcal{I}_{j r}(\mathbf{r})=\int d \mathbf{r}^{\prime} \psi_{j}^{*}\left(\mathbf{r}^{\prime}\right) V\left(\mathbf{r}, \mathbf{r}^{\prime}\right) \psi_{r}\left(\mathbf{r}^{\prime}\right)
$$

where $V\left(\mathbf{r}, \mathbf{r}^{\prime}\right)$ is the two body interaction potential.

\subsection{Observables in second quantization}

Like in first quantization, an observable is a self adjoint operator. Expectation values of an observable $\mathcal{A}$ can be computed using

$$
\langle\mathcal{A}\rangle=\operatorname{Tr}\{\rho \mathcal{A}\}
$$

where $\rho$ is the matrix representation of the density operator of the system. Both $\rho$ and $\mathcal{A}$ can be time dependent so even in a time dependent system, calculating expectation values is straightforward if the matrix of the density operator $\rho$ is known.

An example of an interesting observables is the charge density $\langle\mathcal{Q}\rangle(\mathbf{r}, t)$ can be calculated using

$$
\mathcal{Q} \equiv-q \psi^{\dagger} \psi=-q \sum_{i j} \psi_{i}^{*}(\mathbf{r}) \psi_{j}(\mathbf{r}) d_{i}^{\dagger} d_{j}
$$

so

$$
\langle\mathcal{Q}\rangle(\mathbf{r}, t)=\operatorname{Tr}\{\rho \mathcal{Q}\}=\sum_{\mu \nu} \sum_{i j} \psi_{i}^{*}(\mathbf{r}) \psi_{j}(\mathbf{r}) \rho_{\mu \nu}\left\langle\mu\left|d_{i}^{\dagger} d_{j}\right| \nu\right\rangle
$$

The current density $\mathbf{j}(\mathbf{r}, t)$ for an electron with charge $-q$ is defined in first quantization as (see Ref. [24, 236-244])

$$
\mathbf{j}=-\frac{q}{2 m}\left\{\Psi^{*}(\boldsymbol{\pi} \Psi)+\left(\boldsymbol{\pi} \Psi^{*}\right) \Psi\right\},
$$




\section{Many-electrons calculations}

where $\Psi(\mathbf{r}, t)$ is the single electron wave function. The second quantization equivalent expression is

$$
\begin{aligned}
\boldsymbol{j} & =-\frac{q}{2 m}\left\{\psi^{\dagger}(\boldsymbol{\pi} \psi)+\left(\boldsymbol{\pi} \psi^{\dagger}\right) \psi\right\} \\
& =-q a_{w} \Omega_{w} \sum_{a b} \operatorname{Re}\left(\begin{array}{c}
-i \psi_{a}^{*}(\mathbf{r}) a_{w} \partial_{x} \psi_{b}(\mathbf{r})-\frac{\omega_{c}}{\Omega_{w}} \psi_{a}^{*}(\mathbf{r}) \frac{y}{a_{w}} \psi_{b}(\mathbf{r}) \\
-i \psi_{i}^{*}(\mathbf{r}) a_{w} \partial_{y} \psi_{j}(\mathbf{r})
\end{array}\right) d_{i}^{\dagger} d_{j} \\
& \equiv-q a_{w} \Omega_{w} \sum_{i j} \operatorname{Re}\left(\mathbf{j}_{i j}\right) d_{i}^{\dagger} d_{j},
\end{aligned}
$$

which gives

$$
\langle\boldsymbol{j}\rangle(\mathbf{r}, t)=a_{w} \Omega_{w} \sum_{\mu \nu i j} \operatorname{Re}\left(\mathbf{j}_{i j}\right) \rho_{\mu \nu}\left\langle\mu\left|\hat{d}_{i}^{\dagger} d_{j}\right| \nu\right\rangle
$$

Finally, the expectation value of the energy of the system with the Hamiltonian $\mathcal{H}$ can be calculated using

$$
\langle E\rangle=\operatorname{Tr}\{\rho \mathcal{H}\} .
$$

For a static Hamiltonian, $\langle E\rangle$ is of course a constant of motion.

\subsection{Many electron Hamiltonian}

For more than one electron, we must take into account the Coulomb interaction between them. We can write the many electron Hamiltonian of the system as

$$
\mathcal{H}_{e} \equiv \mathcal{H}_{e}^{0}+\mathcal{H}_{C}
$$

where $\mathcal{H}_{C}$ contains only the contribution from the Coulomb interaction. The purpose of the subscript $e$ is to make a distinction between the electronic part of the Hamiltonian and the photon part, which will be introduced in section 4. Using the formalism in 3.1 we obtain

$$
\mathcal{H}_{e}^{0}=\sum_{i, j}\left\langle i\left|H_{0}\right| j\right\rangle d_{i}^{\dagger} d_{j}=\sum_{i} E_{i} d_{i}^{\dagger} d_{i}
$$

where $\mathcal{H}_{0}$ is the central system Hamiltonian in first quantization. As for the Coulomb interaction term we have

$$
\mathcal{H}_{C}=\frac{1}{2} \sum_{i j r s}\left\langle i j\left|V_{C}\right| r s\right\rangle d_{i}^{\dagger} d_{j}^{\dagger} d_{s} d_{r}
$$


where $V_{C}$ is the Coulomb potential given by

$$
V_{C}\left(\mathbf{r}, \mathbf{r}^{\prime}\right)=\frac{q^{2} / 4 \pi \varepsilon}{\left|\mathbf{r}-\mathbf{r}^{\prime}\right|+\eta}
$$

The purpose of $\eta$ in (3.26) is to prevent the singularity where $\mathbf{r}=\mathbf{r}^{\prime}$. In taking the limit $\eta \rightarrow 0^{+}, \mathcal{H}_{C}$ is nevertheless well defined (for derivation see appendix B).

Plugging (3.26) into (3.25) we get

$$
\left\langle i j\left|V_{C}\right| r s\right\rangle=\int \psi_{i}^{*}(\mathbf{r}) \mathcal{I}_{j r}(\mathbf{r}) \psi_{s}(\mathbf{r}) \mathrm{d} \mathbf{r} .
$$

where

$$
\mathcal{I}_{j r}(\mathbf{r})=\int \psi_{j}^{*}\left(\mathbf{r}^{\prime}\right) \frac{q^{2} / 4 \pi \varepsilon}{\left|\mathbf{r}-\mathbf{r}^{\prime}\right|+\eta} \psi_{r}\left(\mathbf{r}^{\prime}\right) \mathrm{d} \mathbf{r}^{\prime} .
$$

In appendix B I show that the transformation

$$
\mathcal{I}_{j r}(\mathbf{r}) \rightarrow \tilde{\mathcal{I}}_{j r}(\mathbf{r}) \equiv \int\left\{\psi_{j}^{*}\left(\mathbf{r}^{\prime}\right)-\psi_{j}^{*}(\mathbf{r})\right\} \frac{q^{2} / 4 \pi \varepsilon}{\left|\mathbf{r}-\mathbf{r}^{\prime}\right|+\eta}\left\{\psi_{r}\left(\mathbf{r}^{\prime}\right)-\psi_{r}(\mathbf{r})\right\} \mathrm{d} \mathbf{r}^{\prime}
$$

leaves $\mathcal{H}_{C}$ unchanged, but rids of us of the convergence problems we had with $\mathcal{I}_{j r}(\mathbf{r})$. Even though the limit $\eta \rightarrow 0^{+}$is well defined in (3.29), we still have to keep $\eta>0$ for numerical reasons. However, we can have $\eta$ much smaller than if we used (3.28) directly.

The matrix elements of of $\mathcal{H}_{e}$ can now be calculated using

$$
\left\langle\mu\left|\mathcal{H}_{e}\right| \nu\right\rangle=\sum_{i} E_{i} d_{i}^{\dagger} d_{i} \delta_{\mu \nu}+\frac{1}{2} \sum_{i j r s}\left\langle i j\left|V_{C}\right| r s\right\rangle\left\langle\mu\left|d_{i}^{\dagger} d_{j}^{\dagger} d_{s} d_{r}\right| \nu\right\rangle,
$$

where $E_{i}$ is the energy of the SES $i$, associated to the eigenfunction $\psi_{i}(\mathbf{r})$. Now that we have an expression for the matrix elements of $\mathcal{H}_{e}$ in the $\{|\mu\rangle\}$ basis we can diagonalize it and find its eigenstates $\mid \mu$ ) and energies $\tilde{E}_{\mu}$. In the diagonalization process we obtain an unitary transformation $\mathcal{V}$ which satisfies

$$
\mid \mu)=\sum_{\nu=1}^{N_{\text {mes }}} \mathcal{V}_{\mu \nu}|\nu\rangle .
$$

This unitary transformation will be used extensively because it is much more efficient to perform calculations in the $\{|\mu\rangle\}$ basis and perform a unitary transformation to $\{\mid \mu)\}$, rather than explicitly calculating and storing the many electron wave functions. For example if we want to compute a matrix representation of an operator $\mathcal{A}$ in the $\{\mid \mu)\}$ basis, we can use

$$
(\mu|\mathcal{A}| \nu)=\left\langle\mu\left|\mathcal{V}^{\dagger} \mathcal{A} \mathcal{V}\right| \nu\right\rangle
$$

Most of the time it will be clear from context how many electrons the state $\mid \mu$ ) contains. However, where needed I denote the $\mu$-th Coulomb interacting eigenstates containing $N_{e}$ electrons as $\left.\mid \mu\right)_{N_{e}}$. For example, the first excited eigenstate containing 3 electrons is denoted as $\mid 2)_{3}$. For one electron we of course have $\left.|\mu\rangle=\mid \mu\right)_{1}$. 



\section{Inclusion of a quantized EM field}

In this section I will explain the theory needed to describe the dynamics of a system where the nanostructure described in section 2 has been placed inside a photon cavity. The cavity modes of the electromagnetic field can be treated as the population of a field oscillator with different Fock states (states with certain number of photons). All calculations are exact, that is I solve the many electron/photon Schrödinger equation explicitly. I will then introduce the much simpler JC-model as well as more refined TLS models and see how they compare with the exact solution.

\subsection{Quantized electromagnetic field in a cavity}

Suppose the electronic nanostructure described in section 2 is placed in a rectangular cavity forming an electromagnetic oscillator with hard-wall boundaries at $-a_{c} / 2<x, y<$ $a_{c} / 2$ and $-d_{c} / 2<z<d_{c} / 2$ with cavity volume $V_{c}=a_{c}^{2} d_{c}$. The proposed electromagnetic oscillator is a single planar rectangular cavity, in which the electronic nanostructure is oriented in the $z=0$ plane with the center at $(x, y)=(0,0)$. In the following, we will consider only transverse electric (TE) modes $\left(E_{z}=0\right)$, where the electric field $\mathbf{E}$ is perpendicular to the direction of propagation. The cavity supplies a monochromatic wave stabilized in the $\mathrm{TE}_{011}$ mode with longitudinally polarized electric field along $\mathrm{x}$-direction, or in the $\mathrm{TE}_{101}$ mode with transversely polarized electric field along y-direction. In the Coulomb gauge, the vector potential of the electromagnetic field takes the form [25, 282286]

$$
\mathbf{A}_{\mathrm{EM}}=A_{\mathrm{EM}}\left(\begin{array}{c}
\hat{\mathbf{e}}_{x} \\
\hat{\mathbf{e}}_{y}
\end{array}\right)\left(a+a^{\dagger}\right)\left(\begin{array}{c}
\cos \left(\frac{2 \pi x}{a_{c}}\right) \\
\cos \left(\frac{2 \pi y}{a_{c}}\right)
\end{array}\right) \cos \left(\frac{2 \pi z}{d_{c}}\right)
$$

with the upper component denoting the $\mathrm{TE}_{011}$ mode and the lower one representing the $\mathrm{TE}_{101}$ mode. The $a$ and $a^{\dagger}$ are bosonic annihilation and creation operators respectively and $\hat{\mathbf{e}}_{x, y}$ are cartesian unit vectors. 


\section{Inclusion of a quantized EM field}

The allowed energy of mode $\mathrm{TE}_{m n p}$ is given by

$$
\hbar \omega_{m n p}=\hbar c \sqrt{\left(\frac{m \pi}{a_{c}}\right)^{2}+\left(\frac{n \pi}{a_{c}}\right)^{2}+\left(\frac{p \pi}{d_{c}}\right)^{2}},
$$

so for the polarizations given in (4.1) we have

$$
\hbar \omega_{101}=\hbar \omega_{011} \equiv \hbar \omega_{p}=\hbar c \sqrt{\frac{\pi^{2}}{a_{c}^{2}}+\frac{\pi^{2}}{d_{c}^{2}}}
$$

where $\omega_{p}$ is the angular frequency of the photon field. We want $\hbar \omega_{p}$ to be on the same order of magnitude as characteristic energies of the system or about $1 \mathrm{meV}$. Assuming $a_{c} \simeq d_{c}$, (4.3) gives $a_{c}, d_{c} \sim 10^{-3} \mathrm{~m}$, which is in the microwave region and much larger than the length of a typical quantum wire. We can use this fact to simplify equation (4.1) by approximating the vector potential by it's value at the cavity's center, giving

$$
\mathbf{A}_{\mathrm{EM}} \simeq A_{\mathrm{EM}}\left(\begin{array}{c}
\hat{\mathbf{e}}_{x} \\
\hat{\mathbf{e}}_{y}
\end{array}\right)\left(a+a^{\dagger}\right) .
$$

The strength of the photon-electron coupling is characterized by $A_{\mathrm{EM}}$ but it is convenient to define $\mathcal{E}_{c} \equiv q A_{\mathrm{EM}} \Omega_{w} a_{w}$ with the unit of energy and rewrite (4.4) as

$$
\mathbf{A}_{\mathrm{EM}} \simeq \frac{\mathcal{E}_{c}}{q \Omega_{w} a_{w}}\left(\begin{array}{c}
\hat{\mathbf{e}}_{x} \\
\hat{\mathbf{e}}_{y}
\end{array}\right)\left(a+a^{\dagger}\right) .
$$

\subsection{The Hamiltonian}

With the addition of an external EM field we can write the total Hamiltonian of the system as

$$
\mathcal{H}=\mathcal{H}_{e}^{0} \otimes \mathbb{1}^{\mathrm{EM}}+\mathcal{H}_{\mathrm{C}} \otimes \mathbb{1}^{\mathrm{EM}}+\mathbb{1}^{\mathrm{e}} \otimes \mathcal{H}_{\mathrm{EM}}+\mathcal{H}_{\mathrm{e}-\mathrm{EM}}
$$

where $\mathcal{H}_{e}^{0}$ is the Hamiltonian of the central system, $\mathcal{H}_{\mathrm{C}}$ is the Coulomb interaction term, $\mathcal{H}_{\mathrm{EM}}$ is the free field photon term and $\mathcal{H}_{\mathrm{e}-\mathrm{EM}}$ contains the electron-photon interaction. The operators $\mathbb{1}^{\mathrm{e}}$ and $\mathbb{1}^{\mathrm{EM}}$ are the unit operators in the electron and photon Fock space respectively. Most of the time they will be omitted as it should be clear which part of Fock space we are working in.

Let's look closer at the central system and electron-photon interaction terms. Assuming the photon field to have a vector potential $\mathbf{A}_{\mathrm{EM}}$ we have (ignoring the parabolic confinement in the $y$-direction)

$$
\mathcal{H}_{\mathrm{s}}+\mathcal{H}_{\mathrm{e}-\mathrm{EM}}=\frac{1}{2 m} \sum_{i j}\left\langle\psi_{i}\left|\left(\mathbf{p}+q \mathbf{A}+q \mathbf{A}_{\mathrm{EM}}\right)^{2}\right| \psi_{j}\right\rangle d_{i}^{\dagger} d_{j} .
$$


Defining $\boldsymbol{\pi} \equiv \mathbf{p}+q \mathbf{A},(4.7)$ becomes

$$
\begin{aligned}
& \frac{1}{2 m} \sum_{i j}\left\langle\psi_{i}\left|\left(\boldsymbol{\pi}+q \mathbf{A}_{\mathrm{EM}}\right)^{2}\right| \psi_{j}\right\rangle d_{i}^{\dagger} d_{j} \\
& =\sum_{i j}\left\langle\psi_{i}\left|\frac{\boldsymbol{\pi}^{2}}{2 m}+\frac{q}{2 m}\left(\boldsymbol{\pi} \cdot \mathbf{A}_{\mathrm{EM}}+\mathbf{A}_{\mathrm{EM}} \cdot \boldsymbol{\pi}\right)+\frac{q^{2}}{2 m} \mathbf{A}_{\mathrm{EM}}^{2}\right| \psi_{j}\right\rangle d_{i}^{\dagger} d_{j} \\
& =\mathcal{H}_{\mathrm{s}}+\mathcal{H}_{\mathrm{e}-\mathrm{EM}}^{(1)}+\mathcal{H}_{\mathrm{e}-\mathrm{EM}}^{(2)},
\end{aligned}
$$

where I have defined

$$
\begin{aligned}
\mathcal{H}_{\mathrm{e}-\mathrm{EM}}^{(1)} & \equiv \sum_{i j}\left\langle\psi_{i}\left|\frac{q}{2 m}\left(\boldsymbol{\pi} \cdot \mathbf{A}_{\mathrm{EM}}+\mathbf{A}_{\mathrm{EM}} \cdot \boldsymbol{\pi}\right)\right| \psi_{j}\right\rangle d_{i}^{\dagger} d_{j} \\
\mathcal{H}_{\mathrm{e}-\mathrm{EM}}^{(2)} & \equiv \sum_{i j}\left\langle\psi_{i}\left|\frac{q^{2}}{2 m} \mathbf{A}_{\mathrm{EM}}^{2}\right| \psi_{j}\right\rangle d_{i}^{\dagger} d_{j}
\end{aligned}
$$

or, equivalently, but perhaps more intuitive

$$
\begin{aligned}
& \mathcal{H}_{\mathrm{e}-\mathrm{EM}}^{(1)}=-\int \boldsymbol{j} \cdot \mathbf{A}_{\mathrm{EM}} \mathrm{d} \mathbf{r} \\
& \mathcal{H}_{\mathrm{e}-\mathrm{EM}}^{(2)}=-\frac{q}{2 m} \int \mathcal{Q}\left|\mathbf{A}_{\mathrm{EM}}\right|^{2} \mathrm{~d} \mathbf{r},
\end{aligned}
$$

which gives $\mathcal{H}_{\mathrm{e}-\mathrm{EM}}=\mathcal{H}_{\mathrm{e}-\mathrm{EM}}^{(1)}+\mathcal{H}_{\mathrm{e}-\mathrm{EM}}^{(2)}$. The above expressions are greatly simplified when the approximation in (4.5) is utilized because then we can pull $\mathbf{A}_{\mathrm{EM}}$ in front of the integral sign. Plugging the approximation for $\mathbf{A}_{\mathrm{EM}}$ in (4.5) into (4.11) and (4.12) we obtain

$$
\mathcal{H}_{\mathrm{e}-\mathrm{EM}}^{(1)} \simeq \mathcal{E}_{c}\left(a+a^{\dagger}\right) \sum_{i j} g_{i j} d_{i}^{\dagger} d_{j}
$$

where $g_{i j}$ is the dimensionless coupling between the electrons and the cavity mode defined by

$$
g_{i j}=\frac{a_{w}}{2 \hbar} \int d \mathbf{r}\left[\psi_{i}^{*}(\mathbf{r})\left\{(\hat{\mathbf{e}} \cdot \boldsymbol{\pi}) \psi_{j}(\mathbf{r})\right\}+\left\{(\hat{\mathbf{e}} \cdot \boldsymbol{\pi}) \psi_{i}(\mathbf{r})\right\}^{*} \psi_{j}(\mathbf{r})\right]
$$

with $\hat{\mathbf{e}} \cdot \boldsymbol{\pi}=e_{x} \pi_{x}+e_{y} \pi_{y}$. For detailed calculations of $g_{i j}$ see appendix A.4. As for $H_{\mathrm{e}-\mathrm{EM}}^{(2)}$ we have

$$
\begin{aligned}
\mathcal{H}_{\mathrm{e}-\mathrm{EM}}^{(2)} & \simeq \frac{q^{2}}{2 m} \mathbf{A}_{\mathrm{EM}}^{2} \sum_{i j}\left\langle\psi_{i} \mid \psi_{j}\right\rangle d_{i}^{\dagger} d_{j}=\frac{q^{2} \mathbf{A}_{\mathrm{EM}}^{2}}{2 m} \sum_{j} d_{j}^{\dagger} d_{j} \\
& =\frac{\mathcal{E}_{c}^{2}}{\hbar \Omega_{w}}\left(a+a^{\dagger}\right)\left(a+a^{\dagger}\right) \mathcal{N}^{e} \\
& =\frac{\mathcal{E}_{c}^{2}}{\hbar \Omega_{w}}\left[\left(a^{\dagger} a+\frac{1}{2}\right)+\frac{1}{2}\left(a^{\dagger} a^{\dagger}+a a\right)\right] \mathcal{N}^{e},
\end{aligned}
$$




\section{Inclusion of a quantized EM field}

where $\mathcal{N}^{e}$ is the number operator in the electron Fock space. An interesting aspect of $\mathcal{H}_{\mathrm{e}-\mathrm{EM}}^{(2)}$ is that it contains no dependence on the photon polarization or geometry of the system. Putting everything together we obtain

$$
\begin{aligned}
\mathcal{H}_{\mathrm{e}-\mathrm{EM}} & =\mathcal{E}_{\mathrm{c}} \sum_{i, j} d_{i}^{\dagger} d_{j} g_{i j}\left(a+a^{\dagger}\right) \\
& +\frac{\mathcal{E}_{\mathrm{c}}^{2}}{\hbar \Omega_{w}} \mathcal{N}_{\mathrm{e}}\left[\left(a^{\dagger} a+\frac{1}{2}\right)+\frac{1}{2}\left(a^{\dagger} a^{\dagger}+a a\right)\right],
\end{aligned}
$$

The first and the second terms in (4.18) contribute, respectively, to the linear and nonlinear optical excitation energy spectra, which will be explored later.

A convenient basis for doing calculation is $\{\mid \mu) \otimes|M\rangle\} \equiv\{|\breve{\mu}\rangle\}$, where $\mid \mu$ ) are eigenstates of the Hamiltonian of the central system including the Coulomb interaction and $|M\rangle$ are eigenstates of the photon number operator with eigenvalue $M$. To obtain the matrix elements of the total Hamiltonian, including the photon field and e-EM interaction we need to calculate $\langle\mu ; M|\mathcal{H}| \nu ; N\rangle$. For the central system plus Coulomb interaction we have

$$
\left\langle\mu ; M\left|\mathcal{H}_{\mathrm{e}} \otimes \mathbb{1}^{\mathrm{EM}}\right| \nu ; N\right\rangle=\left(\mu\left|\mathcal{H}_{\mathrm{e}}\right| \nu\right)\left\langle M\left|\mathbb{1}^{\mathrm{EM}}\right| N\right\rangle=\tilde{E}_{\mu} \delta_{\mu \nu} \delta_{M N}
$$

where $\tilde{E}_{\mu}$ is the energy of the purely electronic state $\mid \mu$ ). For the free field photon Hamiltonian we have

$$
\left\langle\mu ; M\left|\mathbb{1}^{\mathrm{e}} \otimes \mathcal{H}_{\mathrm{EM}}\right| \nu ; N\right\rangle=\left(\mu\left|\mathbb{1}^{\mathrm{e}}\right| \nu\right)\left\langle M\left|\hbar \omega_{p} a^{\dagger} a\right| N\right\rangle=N \hbar \omega_{p} \delta_{\mu \nu} \delta_{M N}
$$

For the first e-EM interaction term we have

$$
\begin{array}{r}
\left\langle\mu ; M\left|\mathcal{H}_{\mathrm{e}-\mathrm{EM}}^{(1)}\right| \nu ; N\right\rangle=\mathcal{E}_{c} \sum_{i j} g_{i j}\left(\mu\left|d_{i}^{\dagger} d_{j}\right| \nu\right)\left\langle M\left|a+a^{\dagger}\right| N\right\rangle \\
=\mathcal{E}_{c} \mathcal{G}_{\mu \nu}\left(\sqrt{N} \delta_{M, N-1}+\sqrt{N+1} \delta_{M, N+1}\right),
\end{array}
$$

where I have defined

$$
\mathcal{G}_{\mu \nu} \equiv \sum_{i j} g_{i j}\left(\mu\left|d_{i}^{\dagger} d_{j}\right| \nu\right)=\sum_{i j} g_{i j}\left\langle\mu\left|\mathcal{V}^{\dagger} d_{i}^{\dagger} d_{j} \mathcal{V}\right| \nu\right\rangle
$$

which we will refer to as the dimensionless geometric coupling (DGC) between states $\mid \mu$ ) and $\mid \nu)$. As for the second term we have

$$
\begin{array}{r}
\left\langle\mu ; M\left|\mathcal{H}_{\mathrm{e}-\mathrm{EM}}^{(2)}\right| \nu ; N\right\rangle=\frac{\mathcal{E}_{c}^{2}}{\hbar \Omega_{w}}\left(\mu\left|\mathcal{N}^{e}\right| \nu\right)\left\langle M\left|\left(a^{\dagger} a+\frac{1}{2}\right)+\frac{1}{2}\left(a a+a^{\dagger} a^{\dagger}\right)\right| N\right\rangle \\
=\frac{\mathcal{E}_{c}^{2}}{\hbar \Omega_{w}} N_{\mu} \delta_{\mu \nu}\left[\left(N+\frac{1}{2}\right) \delta_{M N}+\frac{1}{2} \sqrt{N(N-1)} \delta_{M, N-2}\right. \\
\left.+\frac{1}{2} \sqrt{(N+1)(N+2)} \delta_{M, N+2}\right]
\end{array}
$$


Finally, the matrix elements of the total Hamiltonian $\langle\mu ; M|\mathcal{H}| \nu ; N\rangle$, are obtained by adding (4.19)-(4.23) together.

The final step is diagonalizing $\mathcal{H}$ and obtaining the allowed energies $\breve{E}_{\mu}$ and the corresponding eigenstates $\mid \breve{\mu}$ ) which are related to $|\breve{\mu}\rangle$ by the unitary transformation

$$
\mid \breve{\mu})=\sum_{\nu} \mathcal{W}_{\mu \nu}|\breve{\nu}\rangle
$$

which is obtained in the diagonalization process.

\subsection{The Jaynes-Cummings model}

To describe the interaction between matter and the photons of a quantized electromagnetic field, the Jaynes-Cummings (JC) model is often applied [26]. The JC-model describes the interaction between a two-level system (TLS) and a single field mode. It is a fundamental model in quantum optics and quantum information science [27]. For a TLS with energy level spacing $\Delta$, coupled with strength $\mathcal{E}_{\mathrm{JC}}$ to a resonator with photon energy $\hbar \omega_{p}$, the JC-model is valid when both the detuning $\delta=\left|\hbar \omega_{p}-\Delta\right|$ is sufficiently small and the light-matter coupling strength is much smaller than the photon energy $\left(\mathcal{E}_{\mathrm{JC}} \ll \hbar \omega_{p}\right)$. There can also be no selection rules preventing transitions between the two chosen states. The dynamics can then be obtained by the JC-model [28] and the energy spectrum can be solved exactly if the rotating wave approximation (RWA) is applied [29].

The JC Hamiltonian in the second quantized form can be written as

$$
\mathcal{H}_{\mathrm{JC}}=\frac{1}{2} \Delta_{i j} \sigma_{z}+\hbar \omega_{p} a^{\dagger} a+\mathcal{E}_{\mathrm{JC}}\left(\sigma_{+}+\sigma_{-}\right)\left(a+a^{\dagger}\right),
$$

where $\Delta_{i j}=E_{j}-E_{i}$ denotes the energy difference between the electron states $|i\rangle$ and $|j\rangle$ which have been chosen as the relevant (active) states for the TLS approximation. The ladder operators appropriate for a two-level approximation $\sigma_{ \pm}$are defined by their action on the active states;

$$
\sigma_{+}|i\rangle=|j\rangle, \sigma_{-}|j\rangle=|i\rangle, \sigma_{+}|j\rangle=\sigma_{-}|i\rangle=0 .
$$

If we put $|i\rangle=\left(\begin{array}{l}1 \\ 0\end{array}\right)$ and $|j\rangle=\left(\begin{array}{l}0 \\ 1\end{array}\right)$ we get $\sigma_{ \pm}=\frac{1}{2}\left(\sigma_{x} \pm i \sigma_{y}\right)$, where $\sigma_{x, y, z}$ are the Pauli matrices. Note that the energies of states $|i\rangle$ and $j\rangle$ are shifted to make them symmetric around the zero energy and it is assumed that $E_{j}>E_{i}$

The counter-rotating terms $\sigma_{+} a^{\dagger}$ and $\sigma_{-} a$ in (4.25) are often omitted by taking the RWA to get an exactly solvable model. However, for our comparison we will keep the counter 
rotating terms and solve (4.25) numerically using the Fock space basis $\{|k\rangle \otimes M\rangle\}$, where $k \in\{i, j\}$. This procedure is very straightforward and only the results will be included in this thesis. Comparison of the JC-model with and without the counter rotating terms has been investigated [30-32], however it should be reexamined and compared with a system where realistic effects are included, such as those stemming from the non-trivial geometry of the nanostructure and an external magnetic-field.

\subsection{Comparison of JC-model with exact model}

\subsubsection{Single electron and no magnetic field}

For one electron, zero magnetic field and assuming a TLS description is applicable (choose $|i\rangle$ and $|j\rangle$ as active states), we have according to (4.15);

$$
\mathcal{H}_{\mathrm{e}-\mathrm{EM}}^{(1)}=\mathcal{E}_{c}\left(a+a^{\dagger}\right)\left(g_{i j} d_{i}^{\dagger} d_{j}+g_{j i} d_{j}^{\dagger} d_{i}\right) .
$$

Here we have used that $g_{i j}=0$ if $i=j$ (see appendix A.4). Now, computing the matrix elements we get

$$
\begin{gathered}
\mathcal{H}_{\mathrm{e}-\mathrm{EM}}^{(1)}=\mathcal{E}_{c}\left(a+a^{\dagger}\right)\left(g_{i j}|i\rangle\left\langle j\left|\left\langle i\left|d_{i}^{\dagger} d_{j}\right| j\right\rangle+g_{i j}^{*}\right| j\right\rangle\langle i|\left\langle j\left|d_{j}^{\dagger} d_{i}\right| i\right\rangle\right) \\
=\mathcal{E}_{c}\left(a+a^{\dagger}\right)\left(g_{i j}|i\rangle\left\langle j\left|+g_{i j}^{*}\right| j\right\rangle\langle i|\right)=\mathcal{E}_{c}\left(a+a^{\dagger}\right)\left(\begin{array}{cc}
0 & g_{i j} \\
g_{i j}^{*} & 0
\end{array}\right)
\end{gathered}
$$

According to equation (A.28), $g_{i j}$ is purely imaginary for $B=0$ so $g_{i j}=-g_{i j}^{*}$. If we assume $\operatorname{Im}\left(g_{i j}\right)$ is positive (being negative would only change the direction of the EM field by $180^{\circ}$ so it has no effect on any observables in a closed system) then $g_{i j}=i\left|g_{i j}\right|$ and

$$
\mathcal{H}_{\mathrm{e}-\mathrm{EM}}^{(1)}=\mathcal{E}_{c}\left(a+a^{\dagger}\right)\left|g_{i j}\right|\left(\begin{array}{cc}
0 & -i \\
i & 0
\end{array}\right)=\left|g_{i j}\right| \mathcal{E}_{c}\left(a+a^{\dagger}\right) \sigma_{y}
$$

The electronic part of the Hamiltonian is simply a $\sigma_{z}$ matrix multiplied by some constant. The rest of the Hamiltonian (free field and $A^{2}$ term) are trivial in the electronic Fock space (simply an unity operator), so we can perform a rotation in the "spin" Hilbert space around the $z$ axis without changing observables and get

$$
\mathcal{H}_{\mathrm{e}-\mathrm{EM}}^{(1)}=\left|g_{i j}\right| \mathcal{E}_{c}\left(a+a^{\dagger}\right) \sigma_{x}=\left|g_{i j}\right| \mathcal{E}_{c}\left(a+a^{\dagger}\right)\left(\sigma^{+}+\sigma^{-}\right) .
$$

This is simply the interaction term in the JC-Hamiltonian in Eq. (4.25) with $\mathcal{E}_{\mathrm{JC}}=\left|g_{i j}\right| \mathcal{E}_{c}$. Note that this result is only exact for one electron and $B=0$. For $B \neq 0, g_{i j}$ remains traceless but individual elements on the diagonal need not be zero. However, the JCmodel does include some of the effects on the magnetic field since it does affect the single electron energy spectrum. 
Using the same TLS approximation as above, we get for the $A^{2}$ term;

$$
\mathcal{H}_{\mathrm{e}-\mathrm{EM}}^{(2)}=\frac{\mathcal{E}_{c}^{2}}{\hbar \Omega_{w}}\left(\left(a^{\dagger} a+\frac{1}{2}\right)+\frac{1}{2}\left(a^{\dagger} a^{\dagger}+a a\right)\right) \otimes \mathbb{1}^{e},
$$

where $\mathbb{1}^{e}$ is a $2 \times 2$ unit matrix. One might be temped to say that we can ignore the $\mathcal{H}_{\mathrm{e}-\mathrm{EM}}^{(2)}$ term for small $\mathcal{E}_{c}$ since it is second order in $\mathcal{E}_{c}$. However, if we apply first order perturbation theory to $\mathcal{H}_{\mathrm{e}-\mathrm{EM}}^{(1)}$, we get zero contribution and the lowest order order contribution is of order $\mathcal{E}_{c}^{2}$. On the other hand, the first order contribution from $\mathcal{H}_{\mathrm{e}-\mathrm{EM}}^{(2)}$ is non-zero. This means that the leading order contribution is of order $\mathcal{E}_{c}^{2}$ for both terms and the question on whether or not we can ignore the $\mathcal{H}_{\mathrm{e}-\mathrm{EM}}^{(2)}$ for small $\mathcal{E}_{c}$ will depend on the magnitude of $\left|g_{i j}\right|$ and $\delta$.

Looking at the equation for $g_{i j}$ in (A.28) we see that it is very sparse for no magnetic field and a small magnetic field does not change that drastically. This means it is easy to find two single electron active states with large coupling while there is minimal coupling with other electronic states. The JC-model can then be a very good approximation as long there is no energy level crossing/anti-crossing between active states and inactive states. As we will see later, things become more complicated for many electron states because of the Coulomb interaction.

\subsubsection{Many electrons and non-zero magnetic field}

Last section we saw that the JC-model can be a good approximation for a single electron and no magnetic field. It will be interesting to see if the same applies to many electron states including magnetic field and Coulomb interaction. Let's begin by applying nondegenerate second order perturbation theory to the $\mathcal{H}_{\mathrm{e}-\mathrm{EM}}^{(1)}$ term in Eq. (4.12),

$$
\breve{E}_{\alpha, M}^{(1),(2)}=\sum_{(\nu, N) \neq(\alpha, M)} \frac{\left|\left(\alpha\left|\left\langle M\left|\mathcal{H}_{\mathrm{e}-\mathrm{EM}}^{(1)}\right| N\right\rangle\right| \nu\right)\right|^{2}}{\left(E_{\alpha}-E_{\nu}\right)+\hbar \omega_{p}(M-N)},
$$

where the super- and subscripts in $\breve{E}_{\alpha, M}^{(1),(2)}$ refer to that it it is the second order correction to energy due to the $\mathcal{H}_{\mathrm{e}-\mathrm{EM}}^{(1)}$ term of state $\left.\mid \alpha\right) \otimes|M\rangle$. Since some of these states are seperated in energy by a small value $\delta$, we have to impose the restraint $\mathcal{E}_{c} \ll|\delta|$ in order for our nondegenerate perturbation description to be valid. If this condition is not satisfied, we would have to resort to perturbation theory for denegerate or almost degenerate states.

We have

$$
\left(\alpha\left|\left\langle M\left|\mathcal{H}_{\mathrm{e}-\mathrm{EM}}^{(1)}\right| N\right\rangle\right| \nu\right)=\mathcal{E}_{c} \mathcal{G}_{\alpha \nu}\left(\sqrt{M} \delta_{N, M-1}+\sqrt{M+1} \delta_{N, M+1}\right)
$$




\section{Inclusion of a quantized EM field}

so

$$
\left|\left(\alpha\left|\left\langle M\left|\mathcal{H}_{\mathrm{e}-\mathrm{EM}}^{(1)}\right| N\right\rangle\right| \nu\right)\right|^{2}=\mathcal{E}_{c}^{2}\left|\mathcal{G}_{\alpha \nu}\right|^{2}\left(M \delta_{N, M-1}+(M+1) \delta_{N, M+1}\right) .
$$

We then get

$$
\begin{aligned}
\breve{E}_{\alpha, M}^{(1),(2)} & =\mathcal{E}_{c}^{2} \sum_{\nu \neq \alpha}\left|\mathcal{G}_{\alpha \nu}\right|^{2}\left(\frac{M}{\Delta_{\nu \alpha}+\hbar \omega_{p}}+\frac{M+1}{\Delta_{\nu \alpha}-\hbar \omega_{p}}\right) \\
& =\mathcal{E}_{c}^{2} \sum_{\nu \neq \alpha}\left|\mathcal{G}_{\alpha \nu}\right|^{2} \frac{\Delta_{\nu \alpha}(2 M+1)+\hbar \omega_{p}}{\Delta_{\nu \alpha}^{2}-\hbar^{2} \omega_{p}^{2}}
\end{aligned}
$$

where $\Delta_{\nu \alpha} \equiv E_{\alpha}-E_{\nu}$. Now put $\hbar \omega_{p}=\left|\Delta_{\alpha \beta}\right|+\delta$ where $|\delta| \ll\left|\Delta_{\alpha \beta}\right|$. In other words, the frequency of the photon field is on resonance between the electronic states $\mid \alpha$ ) and $\mid \beta$ ) with detuning $\delta$. We then obtain

$$
\begin{aligned}
\breve{E}_{\alpha, M}^{(1),(2)} & =\mathcal{E}_{c}^{2} \sum_{\nu \neq \alpha}\left|\mathcal{G}_{\alpha \nu}\right|^{2} \frac{\left|\Delta_{\alpha \beta}\right|-\Delta_{\alpha \nu}(2 M+1)+\delta}{\Delta_{\alpha \nu}^{2}-\Delta_{\alpha \beta}^{2}-2\left|\Delta_{\alpha \beta}\right| \delta-\delta^{2}} \\
& \simeq \mathcal{E}_{c}^{2} \sum_{\nu \neq \alpha}\left|\mathcal{G}_{\alpha \nu}\right|^{2} \frac{\left|\Delta_{\alpha \beta}\right|-\Delta_{\alpha \nu}(2 M+1)+\delta}{\Delta_{\alpha \nu}^{2}-\Delta_{\alpha \beta}^{2}-2\left|\Delta_{\alpha \beta}\right| \delta} .
\end{aligned}
$$

It is tempting to ignore $\delta$ in the numerator in Eq. (4.36) since $|\delta| \ll\left|\Delta_{\alpha \beta}\right|$ but we can run into trouble when $M=0$ and $\left|\Delta_{\alpha \beta}\right|=\Delta_{\alpha \beta}$. This only applies to the ground state in the two level approximation, that is the state $\mid \alpha) \otimes|0\rangle$ if $E_{\alpha}<E_{\beta}$ or $\left.\mid \beta\right) \otimes|0\rangle$ if $E_{\alpha}>E_{\beta}$. I will treat this special case later. For now let's assume we can ignore the $\delta$ in the numerator in Eq. (4.36). Since $\delta$ is small, we expect the term when $\nu=\beta$ in the sum in Eq. (4.36) to dominate. So

$$
\breve{E}_{\alpha, M}^{(1),(2)} \simeq \mathcal{E}_{c}^{2}\left|\mathcal{G}_{\alpha \beta}\right|^{2} \frac{\left|\Delta_{\alpha \beta}\right|-\Delta_{\alpha \beta}(2 M+1)}{-2\left|\Delta_{\alpha \beta}\right| \delta}=\left\{\begin{array}{cl}
-\left|\mathcal{G}_{\alpha \beta}\right|^{2} \frac{\mathcal{E}_{c}^{2}}{\delta}(M+1) & \text { if } \alpha>\beta \\
\left|\mathcal{G}_{\alpha \beta}\right|^{2} \frac{\mathcal{E}_{c}^{2}}{\delta} M & \text { if } \alpha<\beta
\end{array}\right.
$$

We can choose $|\delta|$ as small as we want as long as $|\delta| \gg \mathcal{E}_{c}$, making the validity of (4.37) dependent on the relationship of $\delta$ and $\mathcal{E}_{c}$. In addition $\left|\mathcal{G}_{\alpha \beta}\right|$ must not be zero.

Now let's go back to the ground state when $M=0$ and assume $\alpha<\beta$ such that the ground state in the TLS approximation is $\mid \alpha) \otimes|0\rangle$. The term in the sum in (4.36) when $\nu=\beta$ is then $-\mathcal{E}_{c}^{2}\left|\mathcal{G}_{\alpha \beta}\right|^{2} /\left(2\left|\Delta_{\alpha \beta}\right|\right)$ which is much smaller than the terms in (4.37). This means that the second order contribution in $\mathcal{E}_{c}$ is much smaller for the TLS ground state than for for other states and therefore we expect the ground state to be flat as a function of $\mathcal{E}_{c}$ for small $\mathcal{E}_{c}$.

Performing similar calculations for the first order correction to the $\mathcal{H}_{\mathrm{e}-\mathrm{EM}}^{(2)}$ term gives

$$
\breve{E}_{\alpha, M}^{(2),(1)}=\frac{\mathcal{E}_{c}^{2}}{\hbar \Omega_{W}} N_{\alpha}(M+1 / 2)
$$


where $N_{\alpha}$ is the number of electrons in the state $\mid \alpha$ ). Condition for ignoring the $A^{2}$ term in a TLS approximation is then according to Eqs. (4.37) and (4.38) roughly

$$
\frac{\left|\mathcal{G}_{\alpha \beta}\right|^{2} \hbar \Omega_{w}}{N_{e} \delta} \equiv \lambda \gg 1
$$

Note that in deriving the above condition, we have used that $\mathcal{E}_{c} \ll|\delta| \ll \Delta_{\alpha \beta}$. Also note that the condition is not valid for the TLS ground state. All we can say about the ground state without performing numerical calculations is that it is insensitive to $\mathcal{E}_{c}$ compared with excited states for small $\mathcal{E}_{c}$.

We have seen that under the right circumstances we can use a TLS approximation and ignore the $A^{2}$ term for many electron states. A natural question is then whether or not we can still use the JC-model. Assuming we can use the states $\mid \alpha$ ) and $\mid \beta$ ) where $E_{\alpha}<E_{\beta}$ as a basis and ignore the rest of the electron Hilbert space as well as the $A^{2}$ term of the interaction Hamiltonian, the matrix representation of $\mathcal{H}_{\mathrm{e}-\mathrm{EM}}^{(1)}$ can be written as

$$
\mathcal{E}_{c}\left(\begin{array}{ll}
\mathcal{G}_{\alpha \alpha} & \mathcal{G}_{\alpha \beta} \\
\mathcal{G}_{\alpha \beta}^{*} & \mathcal{G}_{\beta \beta}
\end{array}\right)\left(a+a^{\dagger}\right) \equiv \mathcal{E}_{c} \mathcal{G}^{\mathrm{JC}}\left(a+a^{\dagger}\right)
$$

This can only be the interaction part of a JC-like Hamiltonian if the diagonal of $\mathcal{G}^{\mathrm{JC}}$ is close to zero. A condition for using the JC-model can then be written as

$$
\left|\mathcal{G}_{\alpha \beta}\right| \gg \max \left(\left|\mathcal{G}_{\alpha \alpha}\right|,\left|\mathcal{G}_{\beta \beta}\right|\right) \text {. }
$$

If the above condition is satisfied, we can approximate $\mathcal{G}^{\mathrm{JC}}$ as a linear combination of $\sigma_{x}$ and $\sigma_{y}$ matrices so we can perform a similar rotation about the "spin" $z$-axis as we did for the single electron case in section 4.4.1 and obtain the JC Hamiltonian

$$
\mathcal{H}_{\mathrm{JC}}=\frac{1}{2} \Delta_{\alpha \beta} \sigma_{z}+\hbar \omega_{p} a^{\dagger} a+\mathcal{E}_{\mathrm{JC}}\left(\sigma^{+}+\sigma^{-}\right)\left(a+a^{\dagger}\right)
$$

where

$$
\mathcal{E}_{\mathrm{JC}}=\left|\mathcal{G}_{\alpha \beta}\right| \mathcal{E}_{c}
$$

It's easy to check that for one electron and no magnetic field, Eq. (4.43) reduces to our previous result in Eq. (4.25). 



\section{Results}

In this section I will present results from numerical calculations. I will begin with results from the electron part of the total Hamiltonian, ignoring the quantized photon field. Next I present the results of the full e-EM model and investigate how the results compare with TLS approximations where the diamagnetic term is omitted. In choosing the range of parameters in which calculations are performed, I rely on results from convergence tests, which are presented in chapter 6.

In all calculations we used a magnetic field strength of $B=0.1 \mathrm{~T}$, parabolic confinement strength $\hbar \Omega_{0}=1.0 \mathrm{meV}$, quantum wire length $L_{x}=300 \mathrm{~nm}$, relative permittivity of GaAs $\varepsilon_{r}=12.4$ and effective mass of an electron in GaAs $m=0.067 m_{e}$, where $m_{e}$ is the free electron mass. All lengths are given in units of $a_{w}$, which for the previous mentioned values of $B, \Omega_{0}$ and $m$, is about $33.5 \mathrm{~nm}$.

\subsection{Electron part}

Before we go into the main results of the electron-photon system, it's useful to list some information about the electron eigenstates $\mid \mu$ ) between which the photon frequency will be on resonance. Figures 5.1, 5.2 and 5.3 show the charge density of the 6 lowest electronic eigenstates for 1, 2 and 3 electrons. Figure 5.4 shows the absolute value of the dimensionless geometric coupling (DGC) between the lowest 6 states for $x$ and $y$ polarization. This information will be important when we compare the exact model to a TLS approximation where the condition in (4.39) must be satisfied. Finally, the energies of the 6 lowest states are listed in table 5.1. These energies are needed tune to photon frequency to be on resonance between two chosen states. Note that all the results listed in figures 5.15.4 and table 5.1 are for an plain quantum wire. By plain, I mean that the single electron Hamiltonian in (2.1) is used without adding any gaussian potential wells/hills. 


\section{Results}

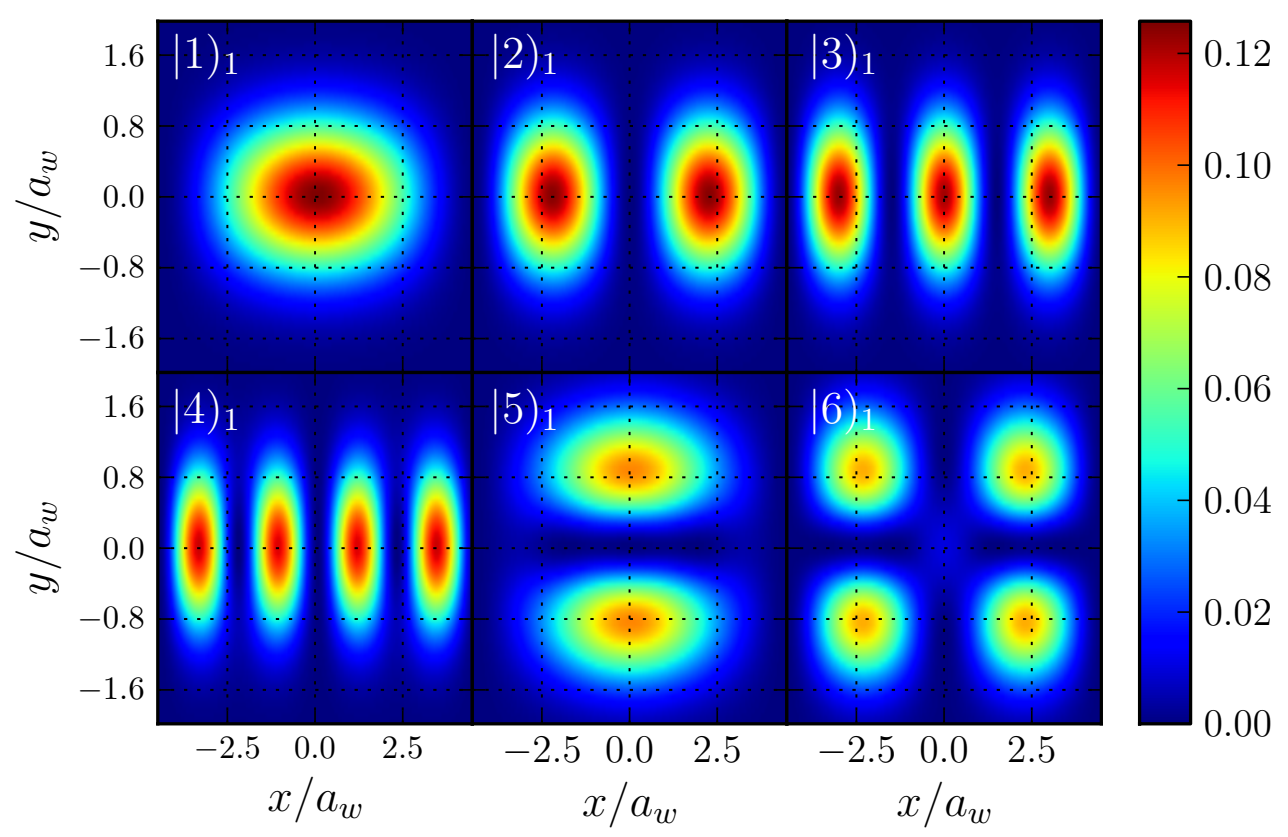

Figure 5.1: Charge density (scaled by $-q$ ) of the lowest 6 Coulomb interacting one electron eigenstates. By comparison with equations (2.6)-(2.7) we can see that the six charge densities correspond roughly to the basis states $\phi_{1}(x) \varphi_{1}(y), \phi_{2}(x) \varphi_{1}(y), \phi_{3}(x) \varphi_{1}(y), \phi_{4}(x) \varphi_{1}(y)$, $\phi_{1}(x) \varphi_{2}(y)$ and $\phi_{2}(x) \varphi_{2}(y)$. By roughly I mean that due to the non-zero magnetic field, $n$ and $m$ are not good quantum numbers.

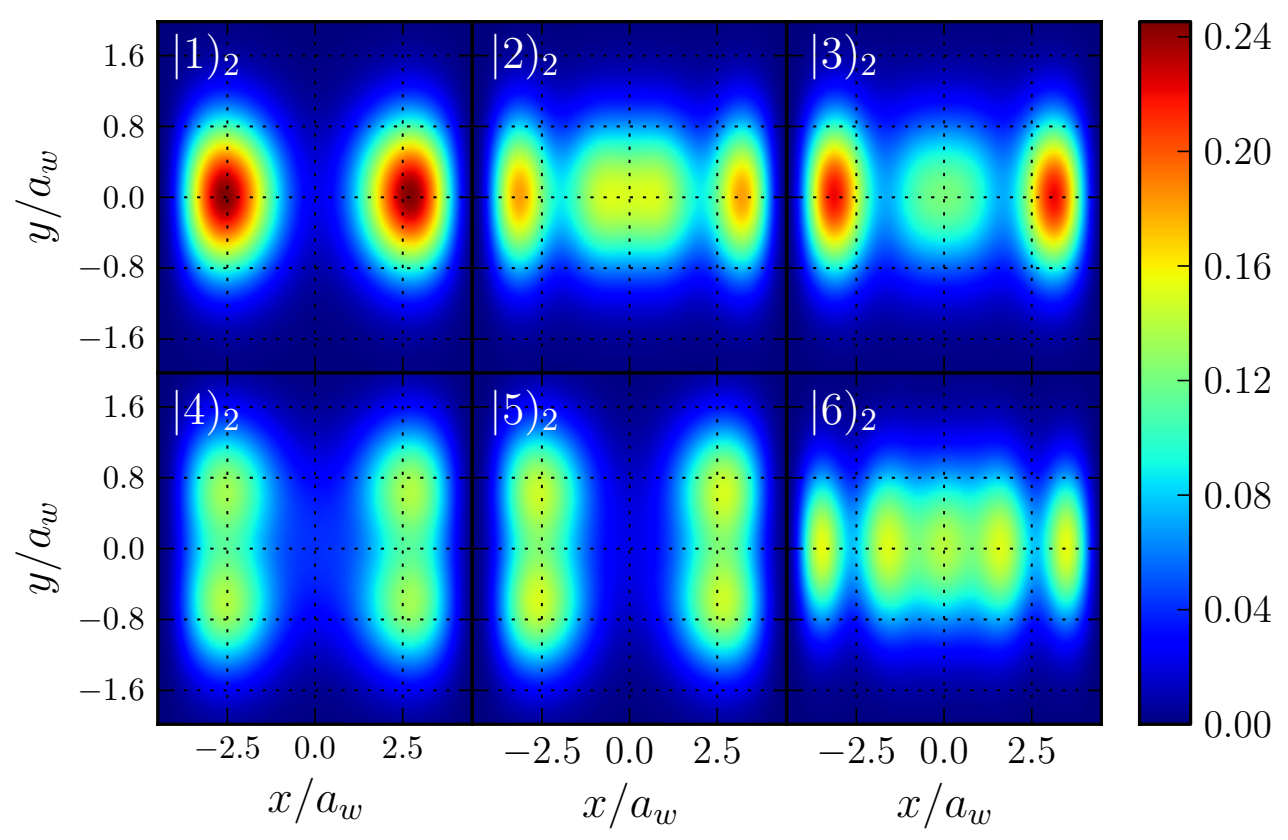

Figure 5.2: Charge density (scaled by $-q$ ) of the lowest 6 Coulomb interacting two electron eigenstates. 


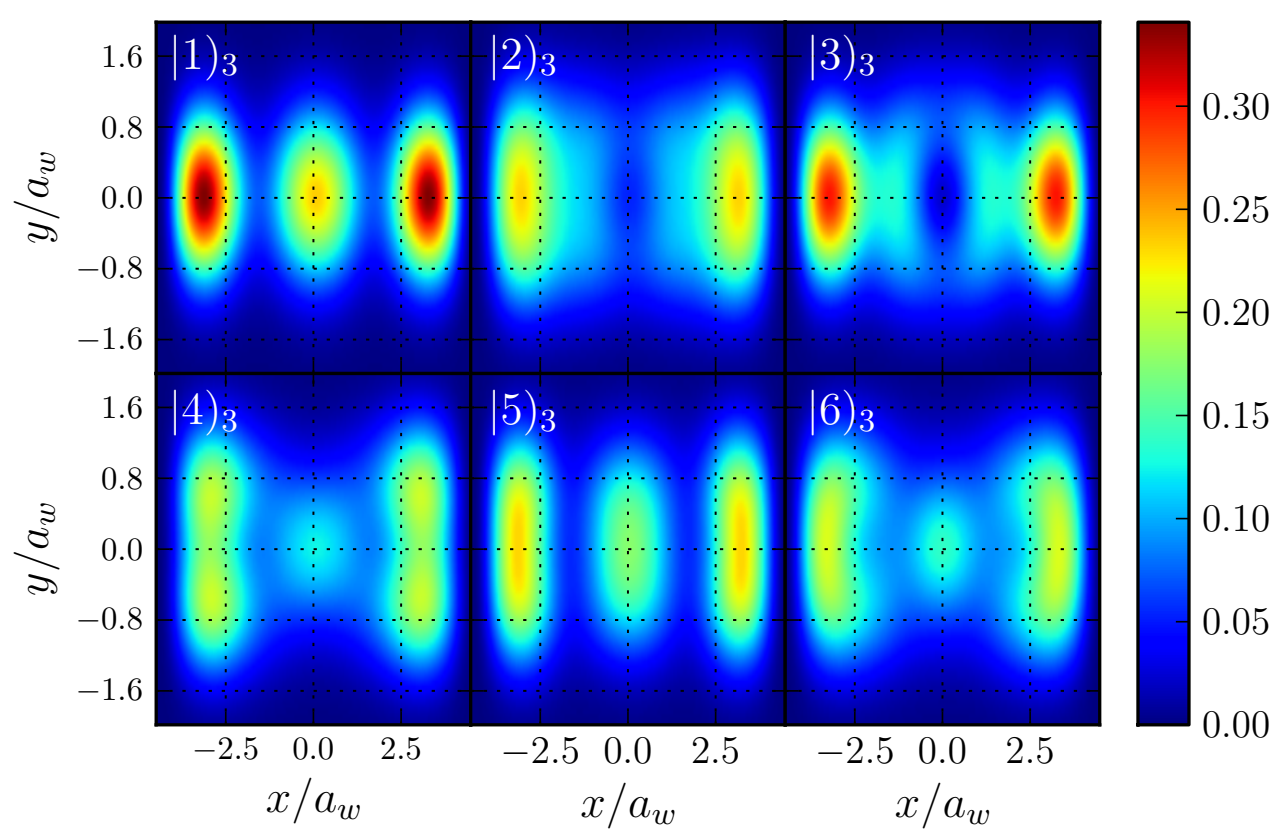

Figure 5.3: Charge density (scaled by $-q$ ) of the lowest 6 Coulomb interacting three electron eigenstates.

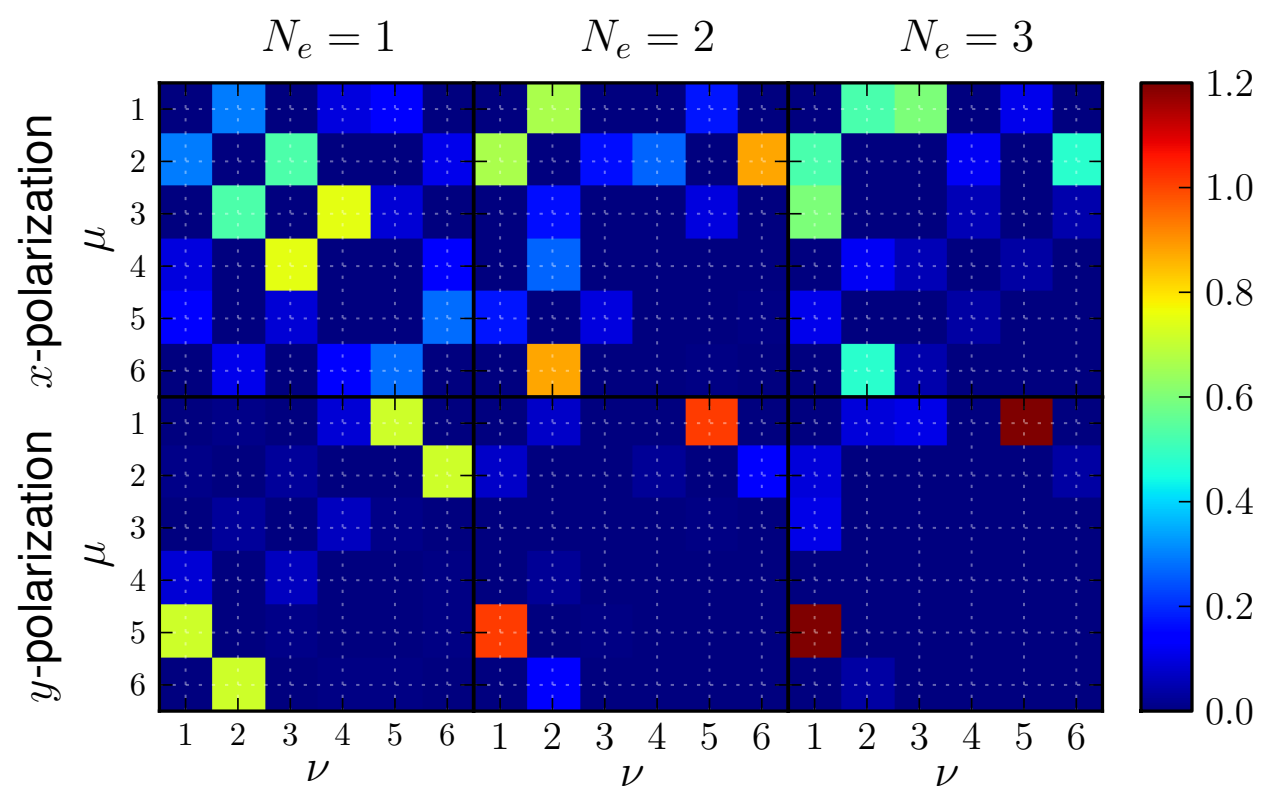

Figure 5.4: DGC strength between the 6 lowest Coulomb interacting eigenstates for both polarizations and 1, 2 and 3 electrons. In other words, it's a color-map plot of the matrix $|\mathcal{G}|$, whose matrix elements are $\left|\mathcal{G}_{\mu \nu}\right|$. From the plot we see that for $x$-polarization, the states most strongly coupled to the ground state are $\left.\left.\mid 2)_{1}, \mid 2\right)_{2}, \mid 2\right)_{3}$ and $\left.\mid 3\right)_{3}$. For $y$-polarization, the state $\mid 5$ ) is most strongly coupled for one, two and three electrons. 
Table 5.1: Energies of the 6 lowest states $\mid \mu$ ) for one, two and three electrons. The energy is in units of meV.

\begin{tabular}{c|cccccc}
$N_{e} \backslash \mu$ & 1 & 2 & 3 & 4 & 5 & 6 \\
\hline 1 & 0.568 & 0.751 & 1.055 & 1.477 & 1.585 & 1.765 \\
2 & 2.215 & 2.726 & 2.857 & 3.183 & 3.230 & 3.330 \\
3 & 5.615 & 6.246 & 6.252 & 6.394 & 6.624 & 6.645 \\
\hline
\end{tabular}

\subsection{Full Model}

\subsubsection{Energy Spectra}

Figures 5.5-5.7 show energy spectra as a function of the coupling strength $\mathcal{E}_{c}$ for both $x$ and $y$-polarization and $1-3$ electrons. In all of these figures, the two active states have strong DGC (large $\left|\mathcal{G}_{\alpha \beta}\right|$ ). Notice how the upper part of the spectra are much more crowded when there is more than one electron. This is most likely the cause of the slow convergence for more than one electron which is covered in section 6 .

It's interesting to compare the results in figures 5.5-5.7 for when the active states have small DGC which is shown in figures 5.8-5.9 for one and two electrons. For the one electron strong DGC case in Fig. 5.5, the $x$-polarization spectrum is relatively smooth, that is there are few abrupt changes or rapid oscillation. However, for the weak DGC case shown in figure 5.8, the spectrum is very chaotic (even after considering the different scales on the $\mathcal{E}_{c}$ axis), exhibiting rapid oscillations and far more crossings/anti-crossings. This is not the case for $y$-polarization since both spectra (weak and strong DGC) show similar behavior for one electron. This is most likely due to the simple parabolic confinement in the $y$-direction. From Fig. 5.9 we can see that the $x$-polarization spectrum is much smoother for two electrons so it seems the Coulomb interaction suppresses the rapid oscillations observed for one electron.

Figures 5.10-5.11 show the importance of including the $A^{2}$ term which is typically omitted in a TLS approximation. In figure 5.10 we can see the comparison of results with and without the $A^{2}$ term of the e-EM interaction Hamiltonian. As can be seen from the figure, omitting the $A^{2}$ term does give accurate results for small $\mathcal{E}_{c}$. We should now consider the condition for ignoring the $A^{2}$ term for small $\mathcal{E}_{c}$ which we derived in Eq. (4.39). For the two cases shown in figure 5.10, we have $\lambda=46.6 \gg 1$ for the $x$-polarization and $\lambda=48.7 \gg 1$ for the $y$-polarization, which is in agreement with our observation that the $A^{2}$ term can be omitted for small $\mathcal{E}_{c}$. Now consider the energy spectra in figure 5.11 where the active states have weak DGC, with $\lambda \simeq 10^{-25}$ for the $x$-polarization and $\lambda \simeq 4 \cdot 10^{-30}$ for the $y$-polarization. We can see that omitting the $A^{2}$ term there is a much worse approximation and is completely unreasonable for the case of $y$-polarization. 

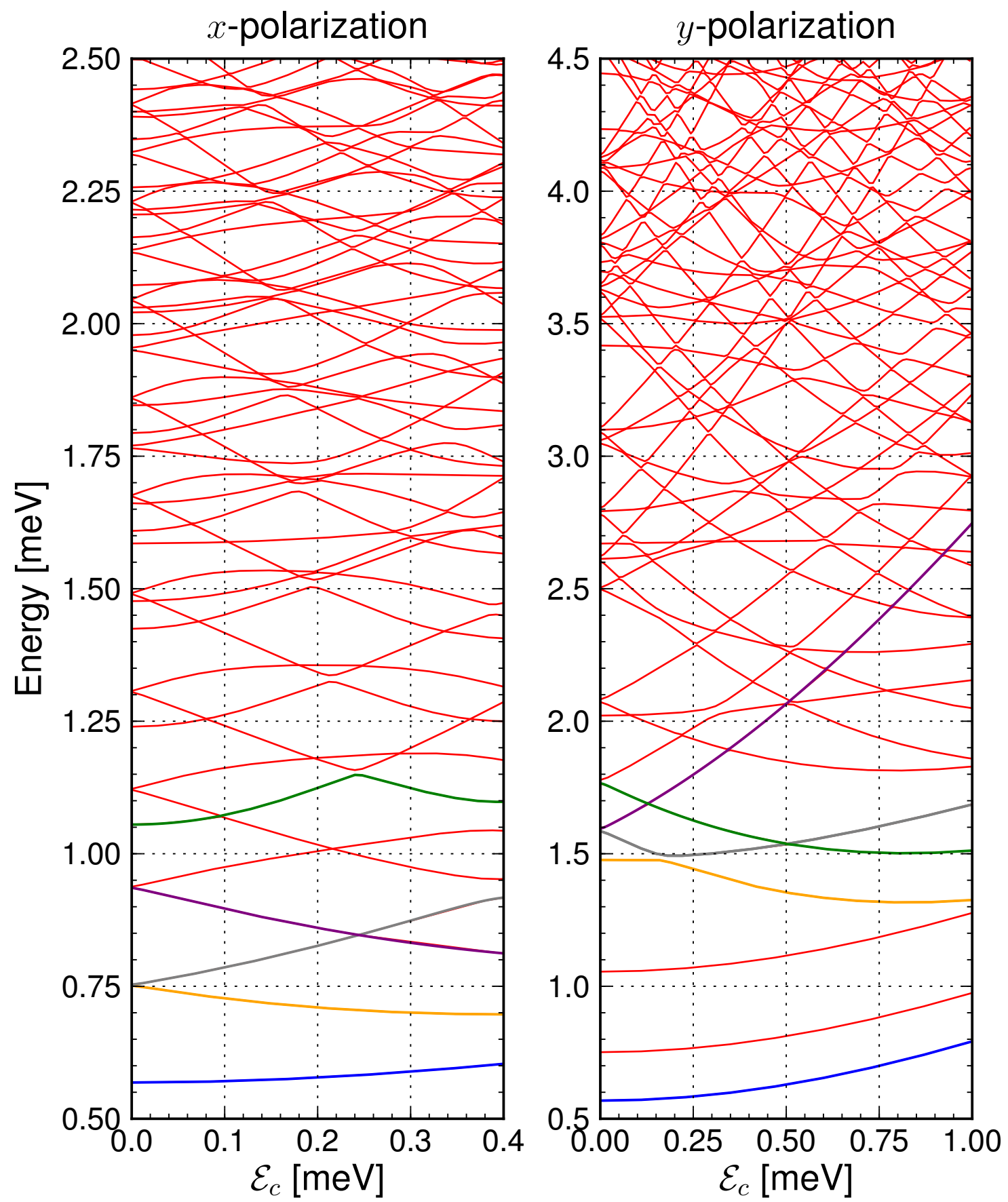

Figure 5.5: Energy spectra for one electron and both $x$ (left) and $y$ (right) polarization. The lowest 64 states are plotted in both cases. For the $x$-polarization, the system is on resonance between the states $\mid 1)_{1}$ and $\left.\mid 2\right)_{1}$ with a DGC strength of $\left|\mathcal{G}_{12}\right|=0.290$ and $\hbar \omega_{p}=0.185 \mathrm{meV}$. For $y$-polarization, the system is on resonance between the states $\mid 1)_{1}$ and $\left.\mid 5\right)_{1}$ with $\left|\mathcal{G}_{15}\right|=0.701$ and $\hbar \omega_{p}=1.03 \mathrm{meV}$. The color coding is used later on to identify states when plotting charge densities in figures 5.12 and 5.13. 

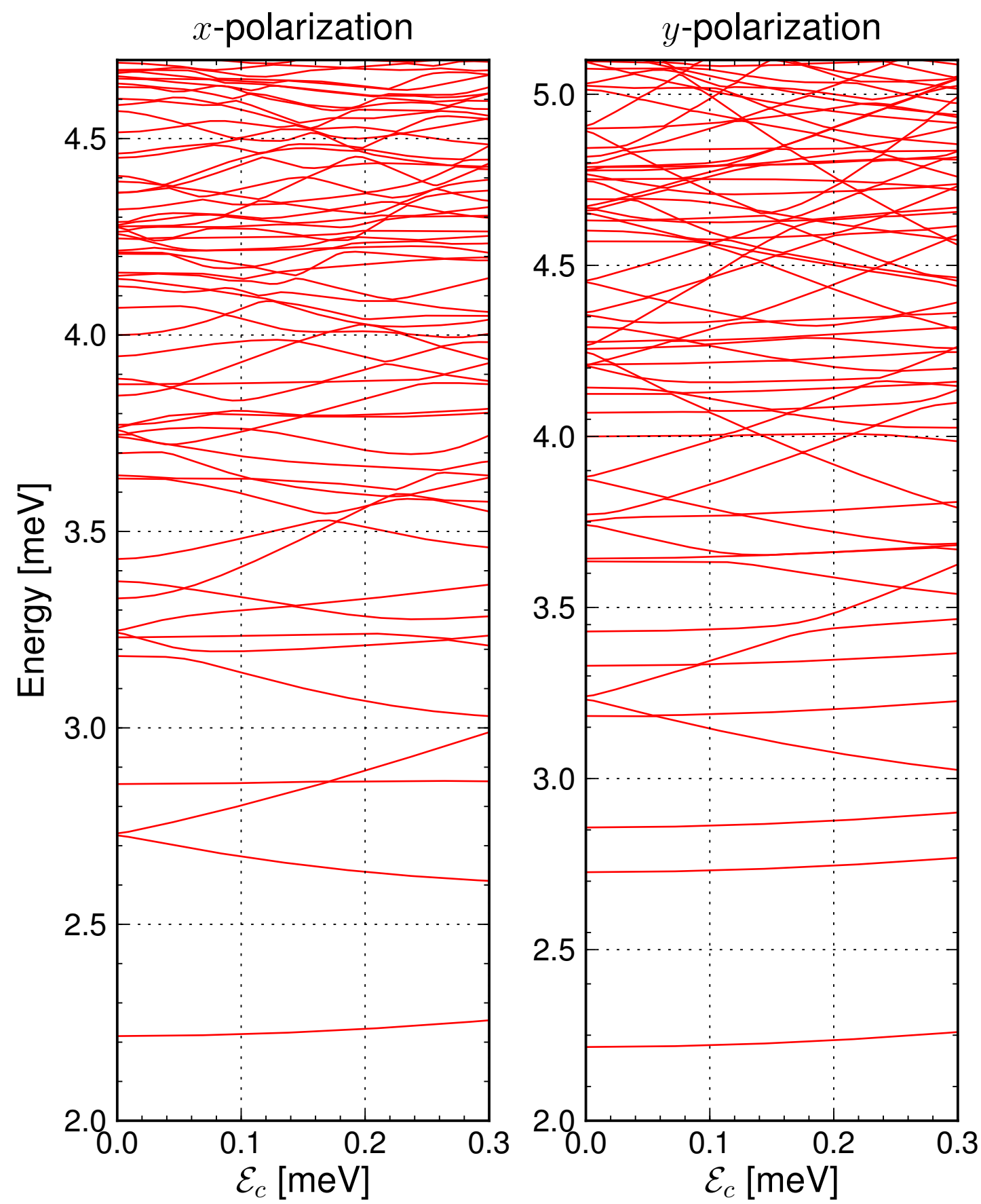

Figure 5.6: Energy spectra for two electrons and both $x$ (left) and $y$ (right) polarization. The lowest 64 states are plotted in both cases. For the $x$-polarization, the system is on resonance between the states $\mid 1)_{2}$ and $\left.\mid 2\right)_{2}$ with a DGC strength of $\left|\mathcal{G}_{12}\right|=0.648$ and $\hbar \omega_{p}=0.516 \mathrm{meV}$. For $y$-polarization, the system is on resonance between the states $\mid 1)_{2}$ and $\left.\mid 5\right)_{2}$ with $\left|\mathcal{G}_{15}\right|=0.987$ and $\hbar \omega_{p}=1.025 \mathrm{meV}$. 

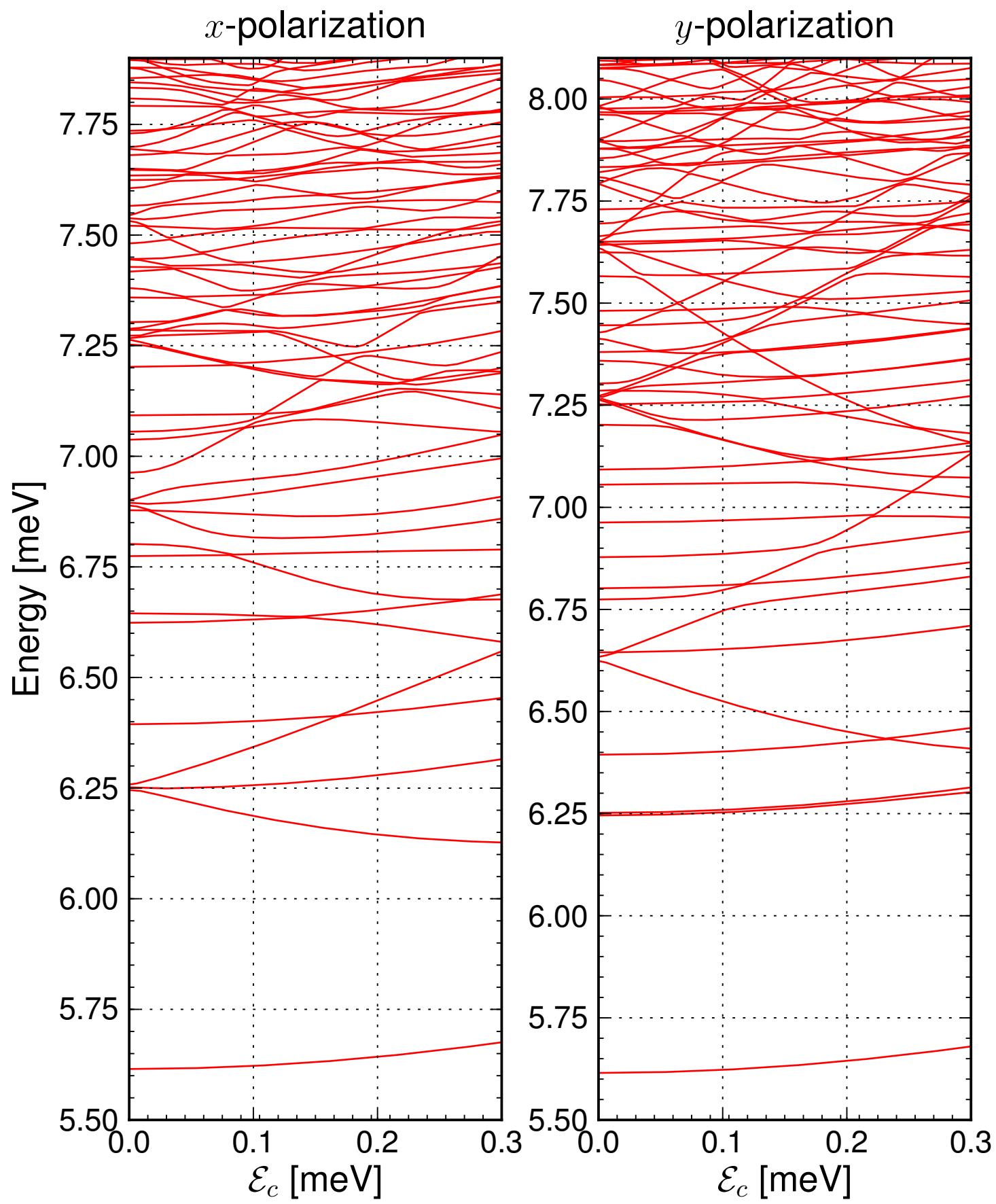

Figure 5.7: Energy spectra for three electrons and both $x$ (left) and $y$ (right) polarization. The lowest 64 states are plotted in both cases. For the $x$-polarization, the system is on resonance between the states $\mid 1)_{3}$ and $\left.\mid 3\right)_{3}$ with a DGC strength of $\left|\mathcal{G}_{12}\right|=0.587$ and $\hbar \omega_{p}=0.643 \mathrm{meV}$. For $y$-polarization, the system is on resonance between the states $\mid 1)_{3}$ and $\left.\mid 5\right)_{3}$ with a DGC strength of $\left|\mathcal{G}_{15}\right|=1.168$ and $\hbar \omega_{p}=1.020 \mathrm{meV}$. 

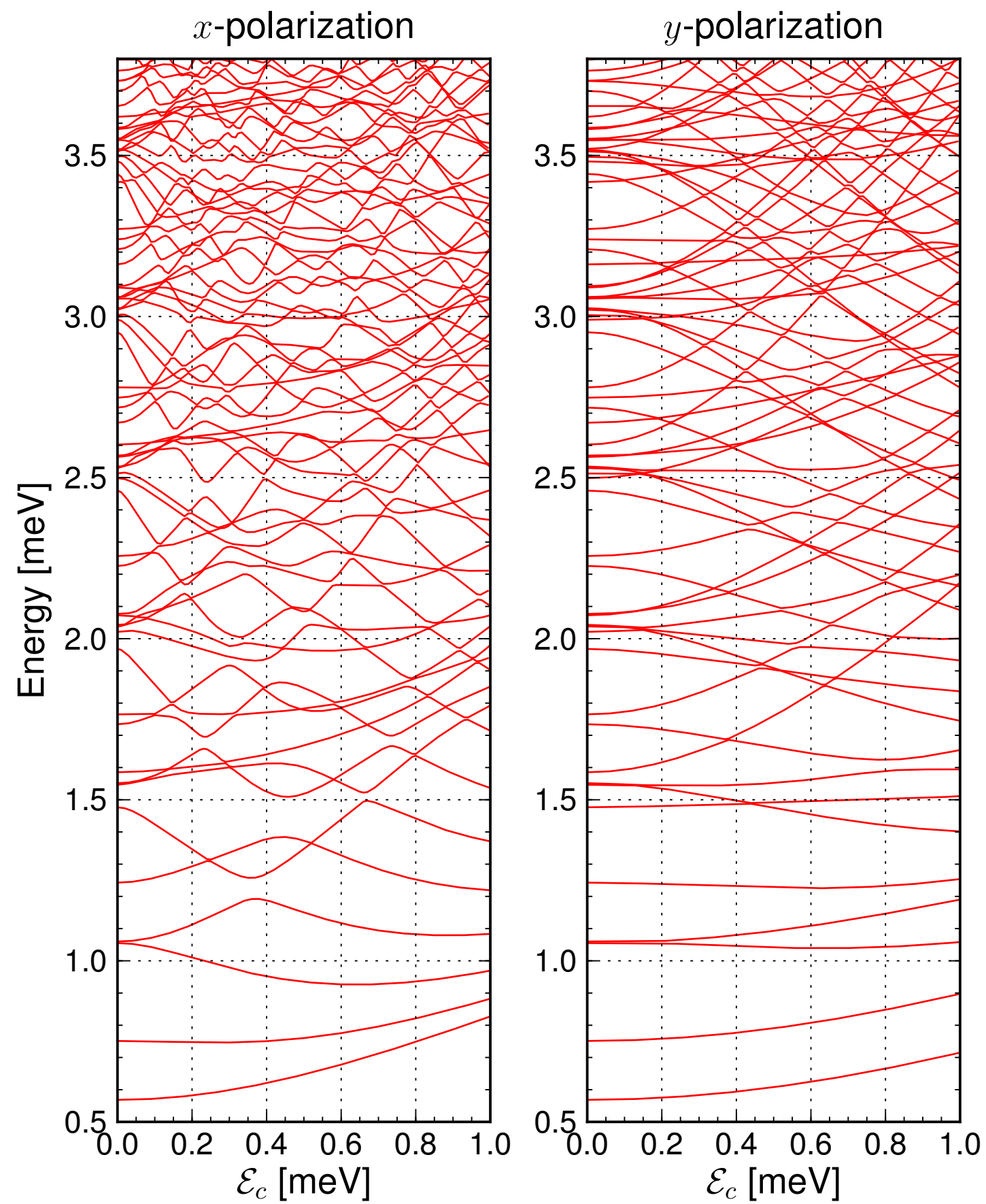

Figure 5.8: Energy spectra for one electron and both $x$ (left) and $y$ (right) polarization. The lowest 64 states are plotted in both cases. The system is on resonance between the weakly coupled states $\mid 1)_{1}$ and $\left.\mid 3\right)_{1}$ with a DGC strength of $\left|\mathcal{G}_{13}\right| \sim 10^{-16}$ and $\hbar \omega_{p}=0.492$ for both polarizations. The spectrum is very chaotic for the $x$-polarization, while the one for the $y$-polarization is relatively smooth. 

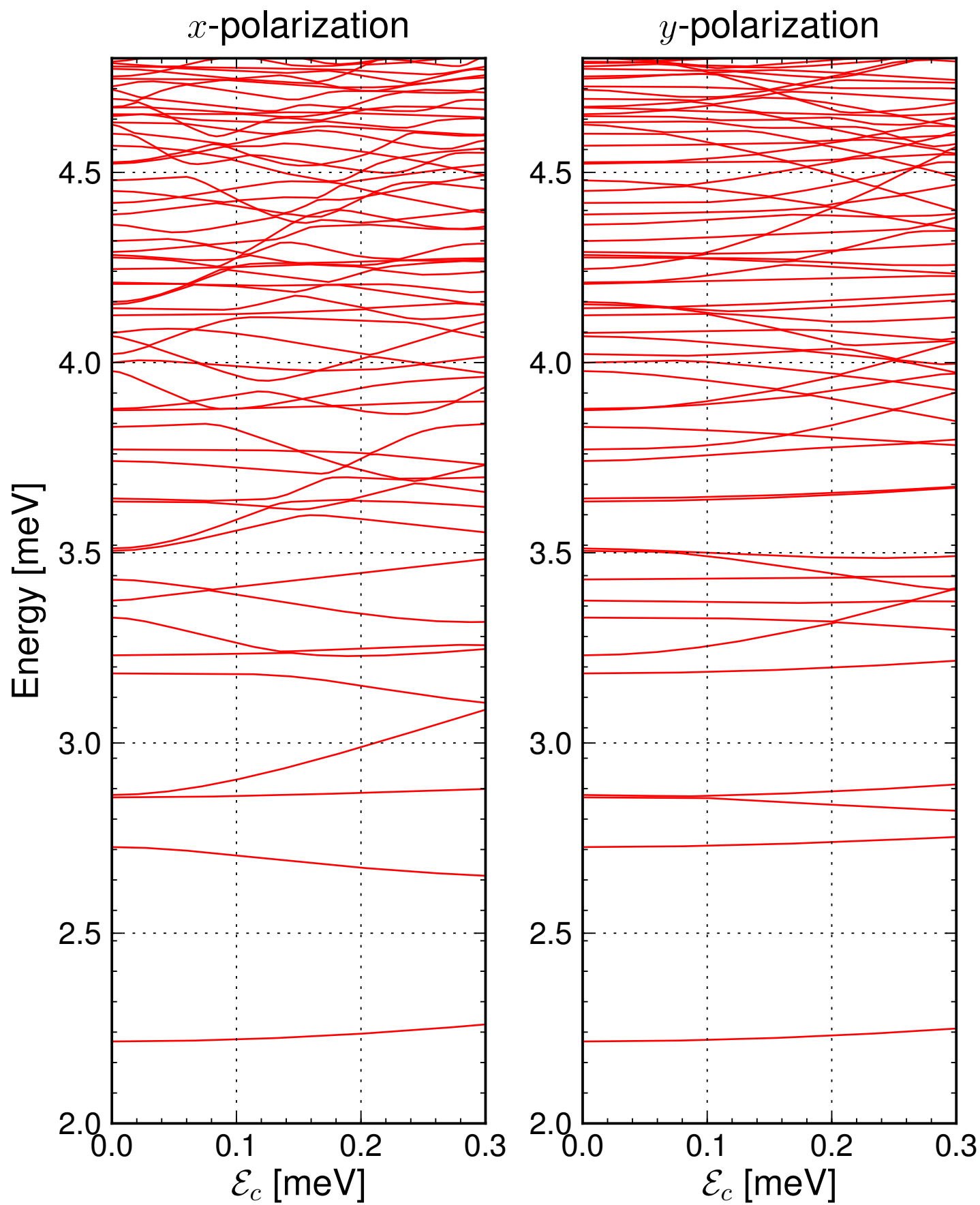

Figure 5.9: Energy spectra for two electrons and both $x$ (left) and $y$ (right) polarization. The lowest 64 states are plotted in both cases. The system is on resonance between the weakly coupled states $\mid 1)_{2}$ and $\left.\mid 3\right)_{2}$ with a DGC strength of $\left|\mathcal{G}_{13}\right| \sim 10^{-13}$ and $\hbar \omega_{p}=0.648$ for both polarizations. Note how smooth the $x$-polarization spectrum is compared with the one electron case in Fig. 5.8. 

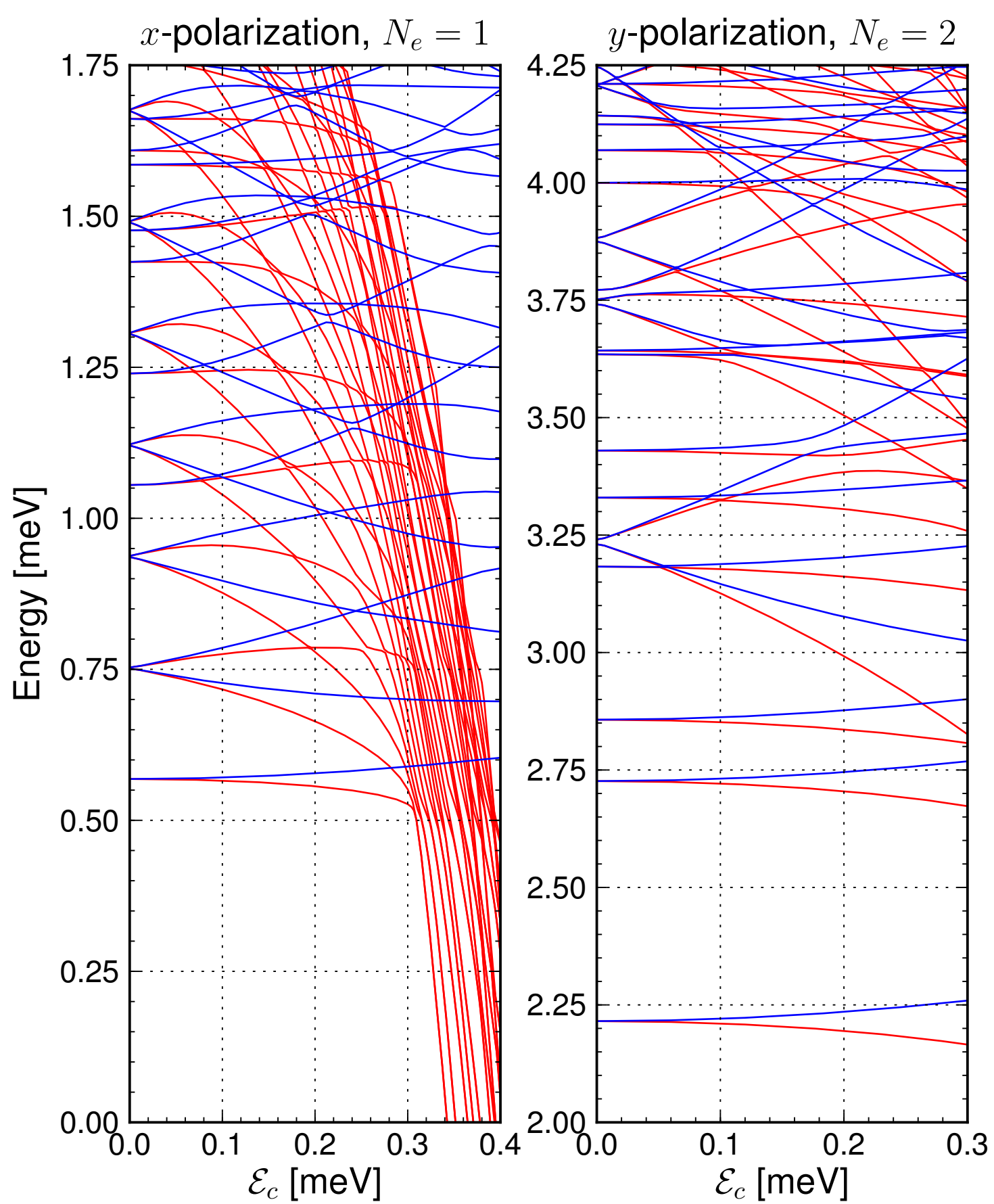

Figure 5.10: Energy spectra for one electron and $x$-polarization (left) and two electrons and $y$ polarization (right). The $A^{2}$ term in the e-EM interaction Hamiltonian is both included (blue) and omitted (red). For the $x$-polarization, the system is on resonance between the states $\mid 1)_{1}$ and $\mid 2)_{1}$ with a DGC strength of $\left|\mathcal{G}_{12}\right|=0.290$ and $\hbar \omega_{p}=0.185 \mathrm{meV}$. For the $y$-polarization, the system is on resonance between the states $\mid 1)_{2}$ and $\left.\mid 5\right)_{2}$ with a DGC strength of $\left|\mathcal{G}_{15}\right|=0.987$ and $\hbar \omega_{p}=1.025 \mathrm{meV}$. As can be seen from the figure, omitting the $A^{2}$ term does give accurate results for small $\mathcal{E}_{c}$, while for large $\mathcal{E}_{c}$ the energy spectrum takes a steep dive downwards. This dive also takes place in the two electron case (right panel), however it can't be seen in the chosen range of $\mathcal{E}_{c}$. There is no physical significance in these dives since the results are highly divergent in those areas as can be seen in Fig. 6.7. 

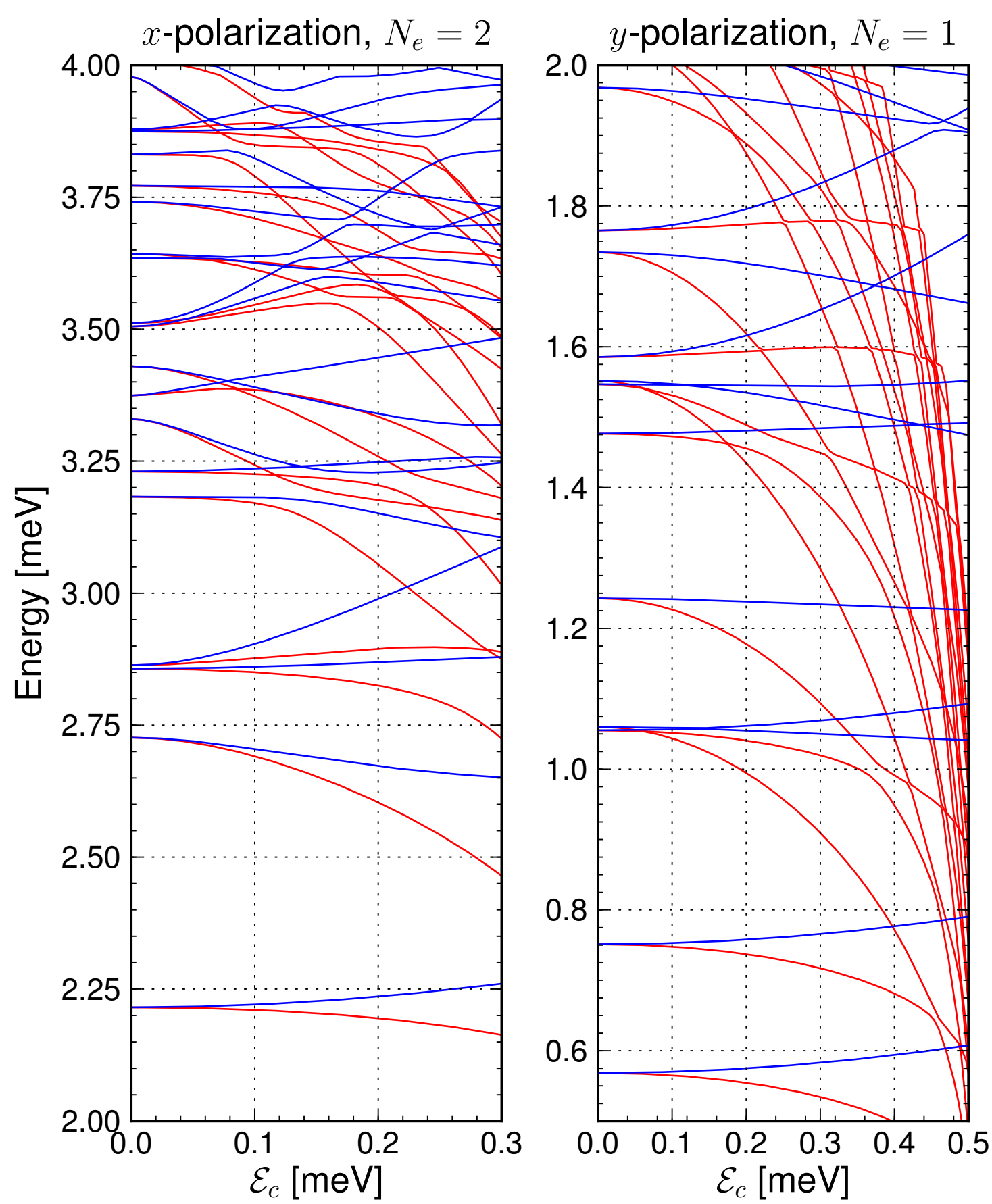

Figure 5.11: Energy spectra for two electrons and $x$-polarization (left) and one electron and $y$ polarization (right). The $A^{2}$ term in the e-EM interaction Hamiltonian is both included (blue) and omitted (red). For the $x$-polarization, the system is on resonance between the weakly coupled states $\mid 1)_{2}$ and $\left.\mid 3\right)_{2}$ with a DGC strength of $\left|\mathcal{G}_{12}\right|=\sim 10^{-13}$ and $\hbar \omega_{p}=0.648 \mathrm{meV}$. For the $y$-polarization, the system is on resonance between the weakly coupled states $\mid 1)_{1}$ and $\left.\mid 3\right)_{1}$ with a DGC strength of $\left|\mathcal{G}_{13}\right| \sim 10^{-13}$ and $\hbar \omega_{p}=0.492 \mathrm{meV}$. Omitting the $A^{2}$ term for the case of $y$-polarization gives very bad results for the majority of the energy curves, even for very small $\mathcal{E}_{c}$. As for the $x$-polarization, we can see that for many of the energy curves, omitting the $A^{2}$ term gives satisfactory results. However, the discrepancy is large for some states, especially for the states $|3\rangle \otimes|0\rangle$ and $|0\rangle \otimes|1\rangle$, the states in which we are most interested in because those states will play a role in the TLS approximation later on. 


\section{Results}

\subsubsection{Charge Density}

It is easy to be overwhelmed with data when plotting charge densities since there are is infinite number of $\mathcal{E}_{c}$ values to choose from and a lot of eigenstates. I will try to choose the most interesting and relevant values. We are mostly interested in the active states and the most interesting areas are around energy crossings and anti-crossings.

To quickly reference eigenstates, the notation $\mid \alpha)|M\rangle\left(\mathcal{E}_{c}\right)$ is used (depending on the context, I will sometimes omit the $\left(\mathcal{E}_{c}\right)$ part). It should be understood as the state we find by starting out at $\mathcal{E}_{c}=0$ and tracing the relevant energy spectrum curve to the right until we reach the desired $\mathcal{E}_{c}$ value. In case of an energy crossing, the smoother (differentiable) curve should be followed. For example, see the purple curve on the right panel in Fig. 5.5. For $\mathcal{E}_{c}=0$, it is the state $\left.\mid 1\right) \mid 1$ ). However, for $\mathcal{E}_{c}>0$, I will still refer to the state $\left.\mid 1\right)|1\rangle$ as the one belonging to the purple curve even though it is no longer an eigenstate. From this discussion it should be clear that when looking at charge density plots, the corresponding energy spectrum from section 5.2.1 should be viewed alongside it.

Figures 5.12 and 5.13 show one electron charge densities for $x$ - and $y$-polarization respectively. From the figures we see that the only area where charge densities changes by a visible amount is near energy anti-crossings. This is well illustrated in the second and

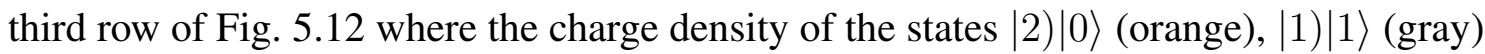
mix due to their anti-crossing at $\mathcal{E}_{c}=0$. Their charge density remains the same when $\mathcal{E}_{c}$ is tuned up until $\mid 1)|1\rangle$ (gray) has another anti-crossing at $\mathcal{E}_{c} \simeq 0.35$. This is also apparent in the fourth row of Fig. 5.13 where the state $\mid 1)|1\rangle$ (purple) has multiple energy crossings in the range $\mathcal{E}_{c} \in[0.2,0.6] \mathrm{meV}$ (see Fig. 5.5) but there is no visible change in the charge density. Another example is the ground state for both polarizations (top row), which have no anti-crossings and the charge density show no visible change.

Charge densities for two or more electrons are omitted in this thesis. The reason is that we need to calculate the charge density in the $\{|\mu| \otimes|M\rangle\}$ basis which means that instead of calculating the simple inner product $\left\langle\mu\left|d_{i}^{\dagger} d_{j}\right| \nu\right\rangle$ in Eq. (3.16), we need to calculate $\left(\mu\left|d_{i}^{\dagger} d_{j}\right| \nu\right)=\left\langle\mu\left|\mathcal{V} d_{i}^{\dagger} d_{j} \mathcal{V}\right| \nu\right\rangle$. This inner product is performed inside a very deeply nested do loop which makes calculating the many electron charge densities computationally unfeasible without drastically reducing the basis size or using some algorithm to select the most important part of $\rho$ and omitting the rest. It is also possible to calculate all possible values of $\left(\mu\left|d_{i}^{\dagger} d_{j}\right| \nu\right)$ beforehand, which results in an array with $N_{\text {mesT }}^{2} \times N_{\text {ses }}^{2}$ elements. For the typical values $N_{\text {mesT }}=200$ and $N_{\text {ses }}=50$ this results in a $\sim 1.5 \mathrm{~GB}$ array which is certainly manageable. 


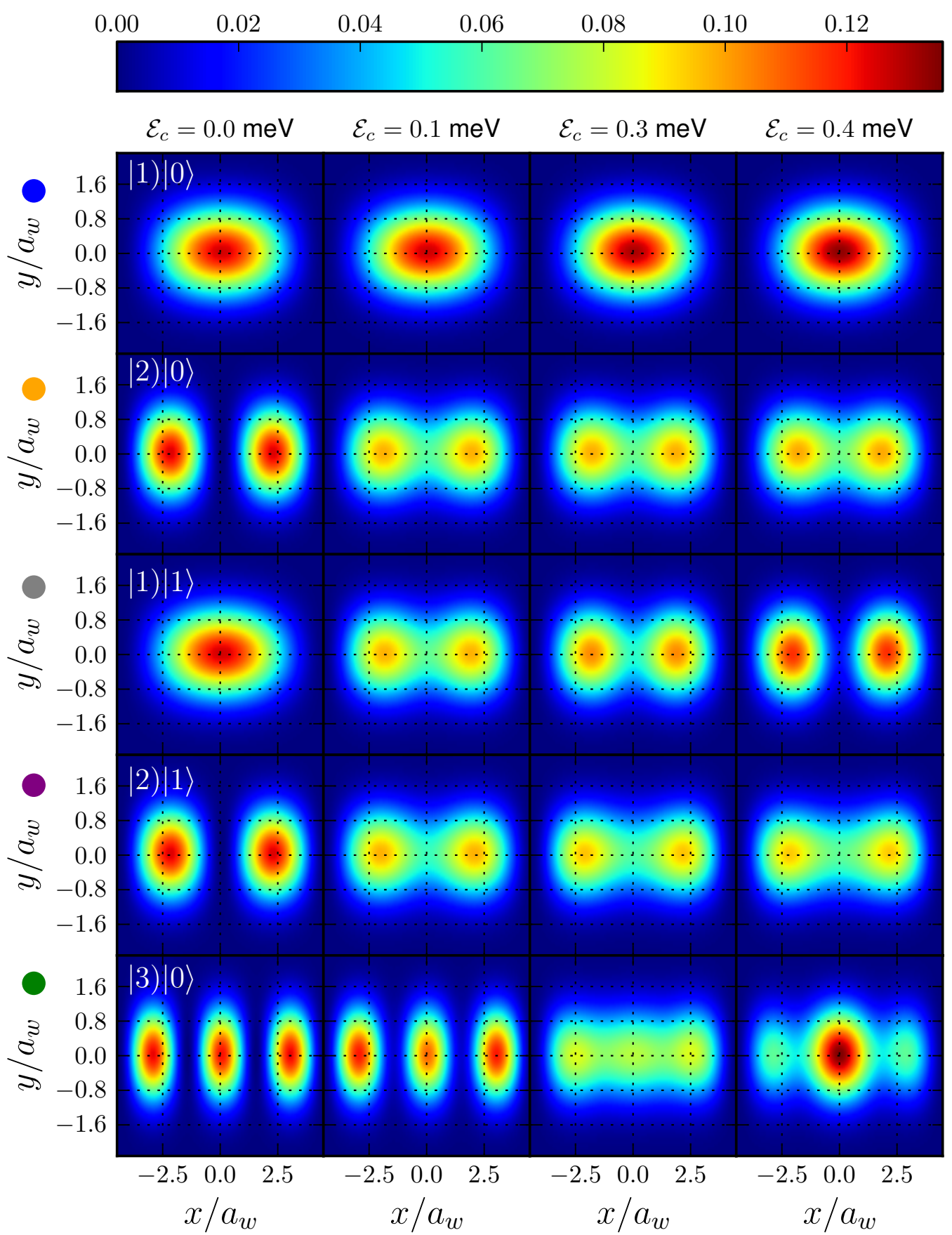

Figure 5.12: Electron charge densities (scaled by $-e$ ) for one electron and $x$-polarization. The system is on resonance between the states $\mid 1)_{1}$ and $\left.\mid 2\right)_{1}$ with a DGC strength of $\left|\mathcal{G}_{12}\right|=0.290$ and $\hbar \omega_{p}=0.185 \mathrm{meV}$. To identify the states, see the color coding on the left panel of Fig. 5.5 and the corresponding color marks on the $y$-axis above. Counting from top to bottom, the plotted states are $\mid 1)|0\rangle$ (blue), $\mid 2)|0\rangle$ (orange), $\mid 1)|1\rangle$ (gray), $\mid 2)|1\rangle$ (purple) and $\mid 3$ ) $|0\rangle$ (green). 


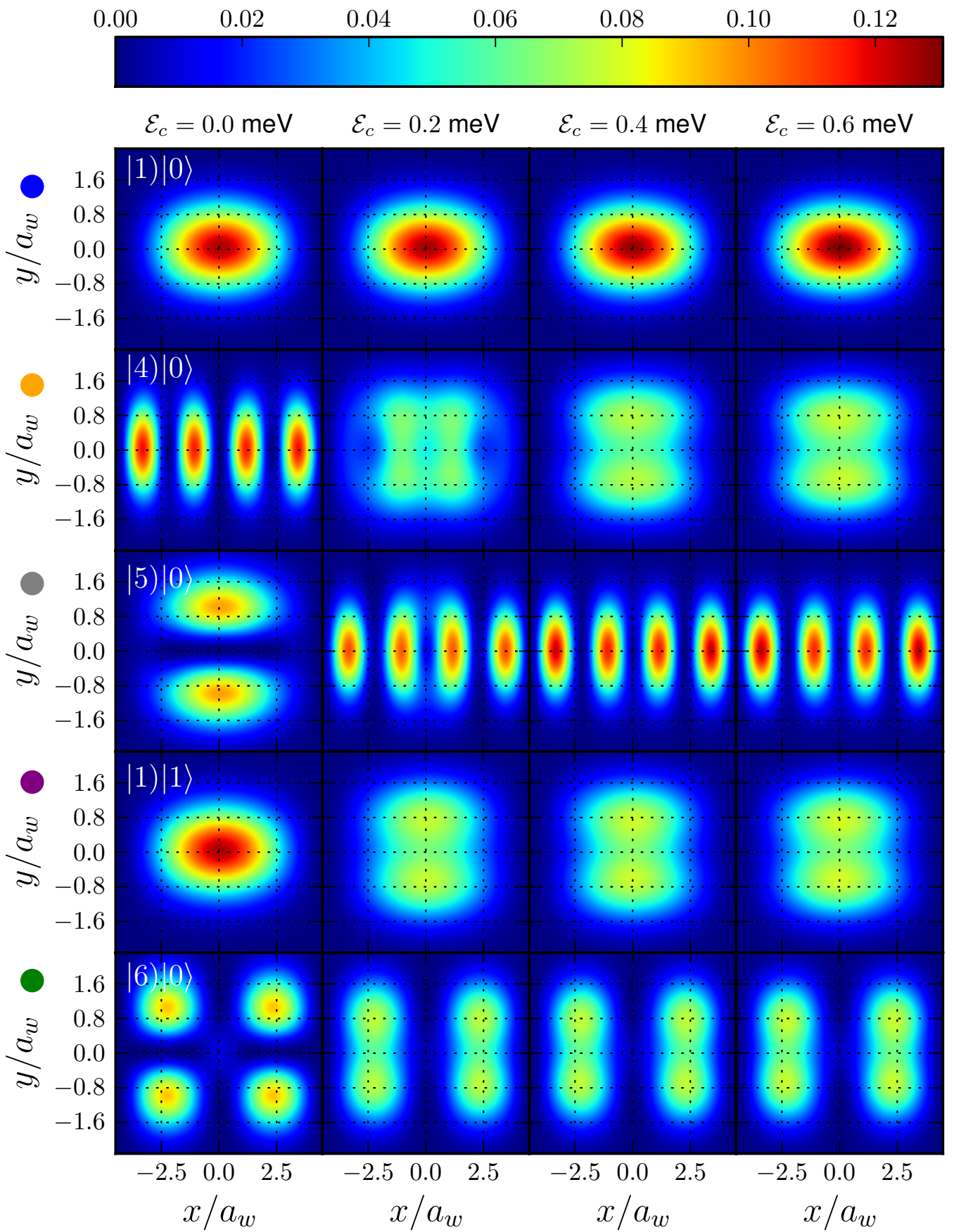

Figure 5.13: Electron charge densities (scaled by $-e$ ) for one electron and $y$-polarization. The system is on resonance between the states $\mid 1)_{1}$ and $\left.\mid 5\right)_{1}$ with a DGC strength of $\left|\mathcal{G}_{15}\right|=0.701$ and $\hbar \omega_{p}=1.03 \mathrm{meV}$. To identify the states, see the color coding on the right panel of Fig. 5.5 and the corresponding color marks on the $y$-axis above. Counting from top to bottom, the plotted states are $\mid 1)|0\rangle$ (blue), $\mid 4)|0\rangle$ (orange), $\mid 5)|0\rangle$ (gray), $\mid 1)|1\rangle$ (purple) and $\mid 6)|0\rangle$ (green). 


\subsection{TLS approximations}

In this section I present results from three different TLS models of varying complexity and compare with results from the exact model. The least complicated one is the JC-model, in which the only input parameters are the energies of the two active states, their DGC strength $\left|\mathcal{G}_{\alpha \beta}\right|$ and the e-EM coupling $\mathcal{E}_{c}$. The other two models which I test are similar to the full model. The difference is that I only use the two active states for the $\{\mid \mu) \otimes|M\rangle\}$ basis, instead of the full $N_{\text {mest }} \simeq 200$ states I use for the full model. Additionally, for one of these two models, I don't include the diamagnetic $A^{2}$ term.

The first thing we need to do in order to investigate the validity of a TLS approximation is to find promising active states to use for the TLS model. As has been covered earlier in this thesis, the active states need to have a strong DGC $\left|\mathcal{G}_{\alpha \beta}\right|$ and be isolated from other energy levels. For $x$-polarization, we can see from Fig. 5.5 that states $\mid 1)_{1}$ and $\left.\mid 2\right)_{1}$ look very promising. This choice gives $\lambda \simeq 46.6 \gg 1$ so we can expect the TLS approximation to be valid, which is confirmed in Fig. 5.14, which shows the $x$-polarization many-body energy spectra as a function of the electron-photon coupling strength for the different models. As expected, the JC results almost coincide with our TLS results not including the $A^{2}$ term. The difference (not visible in Fig. 5.14) between the two curves is due to effects of the external magnetic field.

When the $A^{2}$ term is included, the energy spectrum manifests a blue-shift. A weaker red shift correction is observed when the higher MBSs are involved in the electron-photon coupling. In the weak coupling regime $\mathcal{E}_{\mathrm{c}}<0.1 \hbar \omega \simeq 0.02 \mathrm{meV}$, the JC-model is approximately valid. When the coupling strength is increased to $\mathcal{E}_{\mathrm{c}} \simeq \hbar \omega \simeq 0.2 \mathrm{meV}$, the ground state energy calculated by the TLS model is still valid. However, the energy of the excited states becomes inaccurate, indicating that the simplified TLS model is no longer a good approximation in the strong coupling regime even though the diamagnetic vector potential $A^{2}$ is included. When the coupling strength $\mathcal{E}_{\mathrm{c}}$ is increased, both the JC- model and the TLS without the $A^{2}$ term predict a decreasing ground state, however by including the $A^{2}$ term within the TLS model the energy increases, in better agreement with our full numerical calculation. 


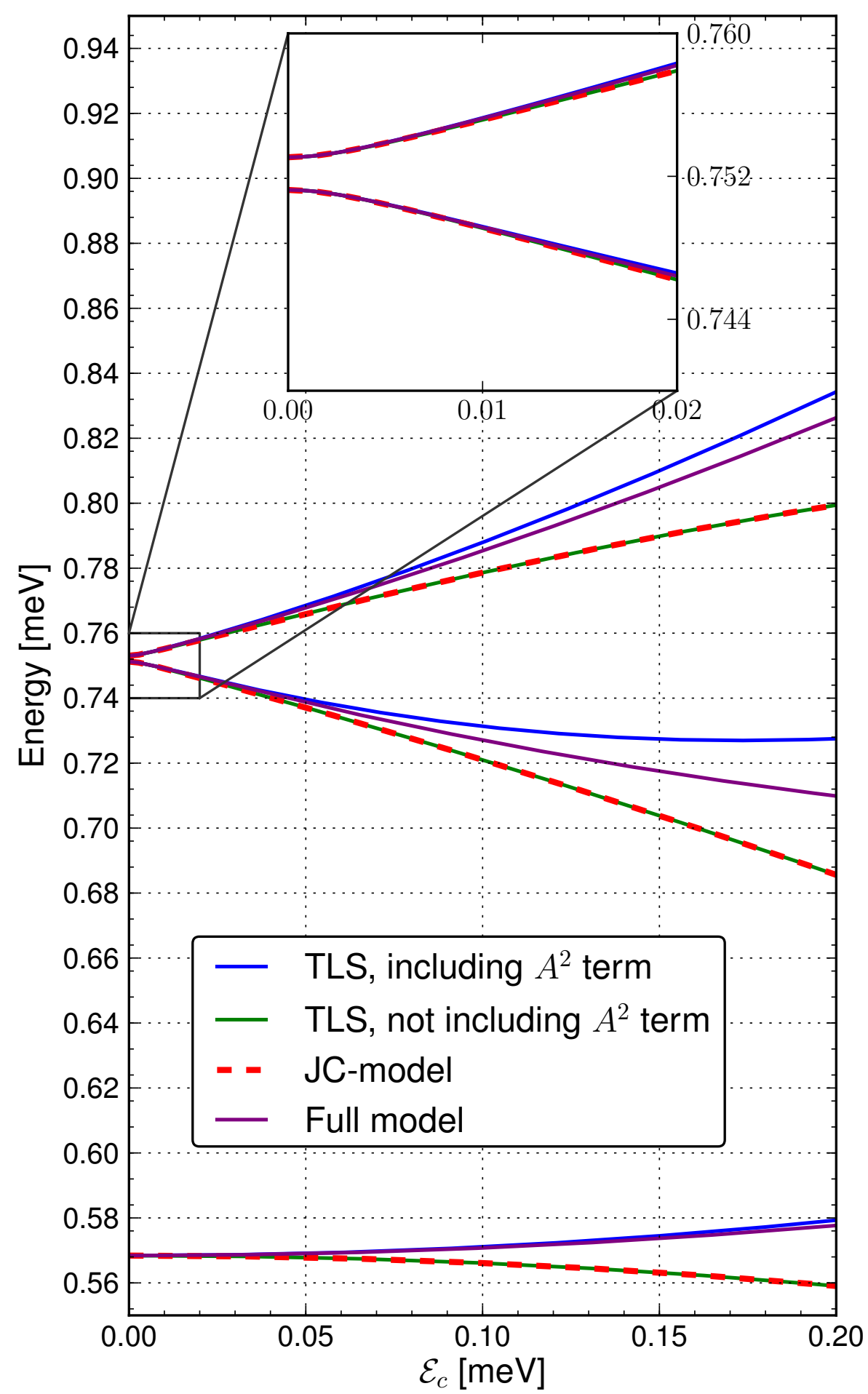

Figure 5.14: Comparison of the many-body energy spectra versus the coupling strength $\mathcal{E}_{\mathrm{c}}$ for the case of one electron and $x$-polarization. The energy spectra's are obtained by the TLS model with (blue) and without (green) the $A^{2}$ term and the JC-model (red-dashed). The TLS model results are compared with the full numerical calculation results for the lowest active levels $\mid 1) \otimes|0\rangle, \mid 2) \otimes|0\rangle$, and $\mid 1) \otimes|1\rangle$ (purple). Other parameters are the same as for the $x$-polarization in Fig. 5.5. 
For $y$-polarization and one electron, we have to choose the states $\mid 1)_{1}$ and $\left.\mid 5\right)_{1}$ since $\mid 5)_{1}$ is the only energetically low state which has a large DGC strength with the one electron ground state $\mid 1)_{1}$. This choice gives $\lambda \simeq 48.7 \gg 1$ so we can expect the TLS approximation to be valid. However, there is clearly an anti-crossing at $\mathcal{E}_{c} \simeq 0.15$ between the states $\mid 5)|0\rangle$ and $\mid 4)|0\rangle$ (see right panel of Fig. 5.5) so we expect the TLS to fail at around $\mathcal{E}_{c} \simeq 0.15$. This is exactly what we see in Fig. 5.15, where we compare the many-body energy spectra as a function of the electron-photon coupling strength $\mathcal{E}_{\mathrm{c}}$ for $y$-polarization. The Figure displays energy spectra calculated using the four models. As with the $x$-polarization, the energy spectrum obtained by the JC-model almost coincides with the TLS result without the $A^{2}$ term. The difference (not visible in Fig. 5.15) is again due to effects of the external magnetic field.

When the $A^{2}$ term is included, the energy spectrum is blue-shifted in the strong coupling regime. When the higher MBSs are involved in the electron-photon coupling (full model) there is good agreement with the TLS including the $A^{2}$ term until inactive states (not included in the two-level approximation) start to have influence, such as the energy anticrossing at $\mathcal{E}_{c} \simeq 0.17 \mathrm{meV}$ shown in Fig. 5.15. Note that the energy crossing at $\mathcal{E}_{c} \simeq$ $0.13 \mathrm{meV}$ between the states $\mid 6)|0\rangle$ and $\mid 1)|1\rangle$ has no visible effect on the active states. In the weak coupling regime $\mathcal{E}_{\mathrm{c}}<0.1 \hbar \omega \simeq 0.1 \mathrm{meV}$, the JC-model is approximately valid. Also note that for $y$-polarization, results are only shown up to $\mathcal{E}_{c} / \hbar \omega_{p} \simeq 0.2$ while for $x$ polarization I go up to $\mathcal{E}_{c} / \hbar \omega_{p} \simeq 1$. This is because there are no inactive states getting in the way for $x$-polarization and thus we can allow ourselves to take the TLS approximation further in $\mathcal{E}_{c}$.

Figures 5.16 and 5.17 show the same comparison for the case of two electrons. Our choice of active states $(\mid 1)_{2}$ and $\left.\mid 2\right)_{2}$ for $x$-polarization and $\left.\mid 1\right)_{2}$ and $\left.\mid 5\right)_{2}$ for $y$-polarization) gives $\lambda \simeq 41.7 \gg 1$ for $x$ polarization and $\lambda \simeq 48.7$ for the $y$-polarization so we can expect the TLS approximation to be valid again. As can be seen from the two figures, the behavior is very similar to the one electron case. Both the results of the JC-model and the TLS without $A^{2}$ term show red shift w.r.t the full model while the TLS with $A^{2}$ term result show a less drastic blueshift. As before, all the TLS results fail around energy anti-crossings.

Finally, Fig. 5.18 shows a TLS comparison when the active states are weakly coupled and condition 4.39 is not satisfied. What is surprising is that the TLS without the $A^{2}$ term is actually more accurate than the one which includes it. The reason for this is is probably that the error originating from neglecting the remainder of the electron Hilbert space partly cancels the error due to the missing $A^{2}$ term. 


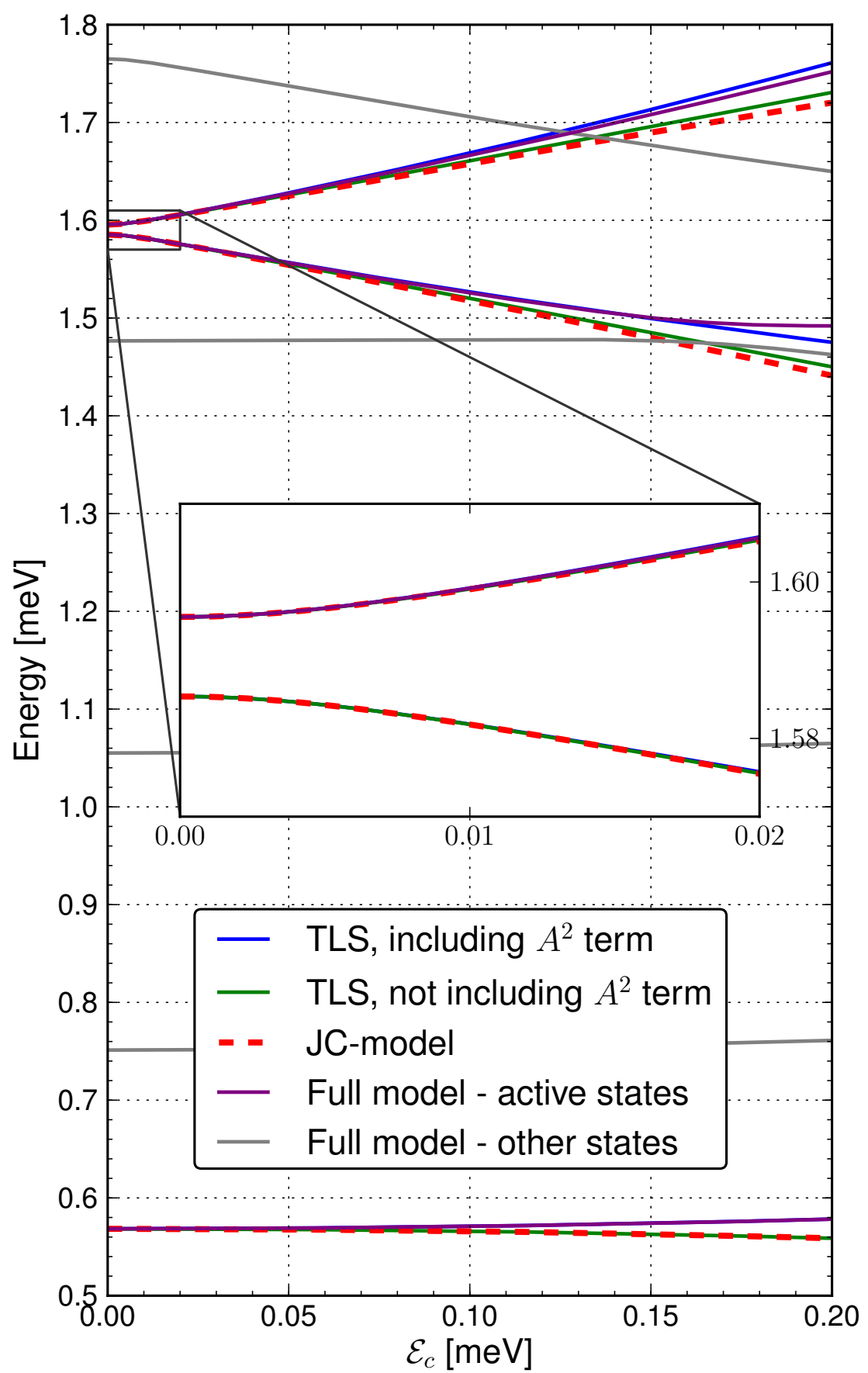

Figure 5.15: Comparison of the many-body energy spectra versus the coupling strength $\mathcal{E}_{\mathrm{c}}$ for the case of one electron and $y$-polarization. These energy states are obtained by TLS model including the $A^{2}$ term (blue), not including the $A^{2}$ term (green) and the JC-model (red-dashed). The TLS model results are compared with the full numerical calculation for the compared lowest active levels $\mid 1)|0\rangle, \mid 5)|0\rangle$ and $\mid 1)|1\rangle$ (purple) as well as inactive levels (gray). Other parameters are the same as for the $y$-polarization in Fig. 5.5. The inset shows the validity of the JC-model in the weak coupling limit. 


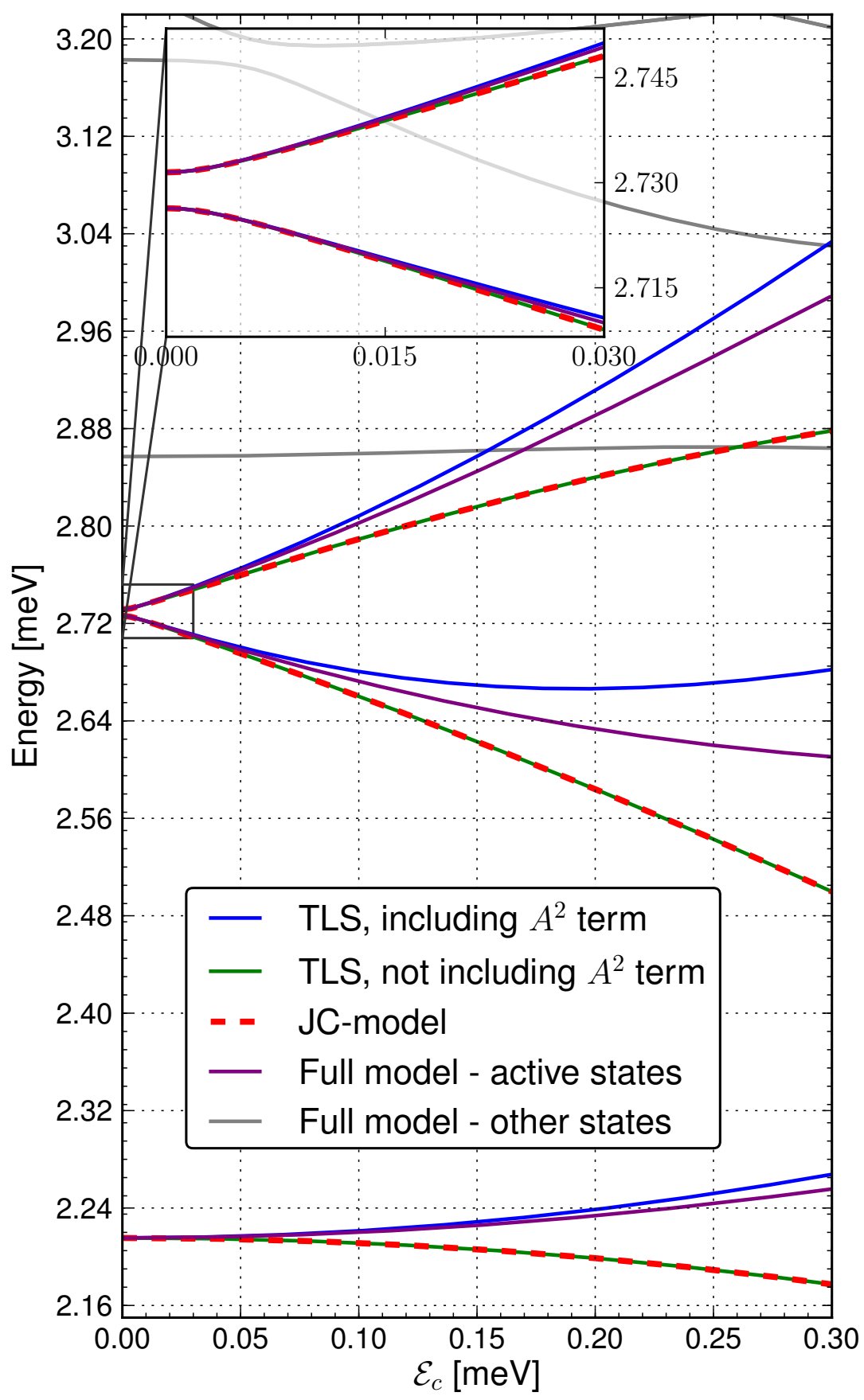

Figure 5.16: Comparison of the many-body energy spectra versus the coupling strength $\mathcal{E}_{\mathrm{c}}$ for the case of two electrons and $x$-polarization. These energy states are obtained by TLS model including the $A^{2}$ term (blue), not including the $A^{2}$ term (green) and the JC-model (red-dashed). The TLS model results are compared with the full numerical calculation for the compared lowest active levels $\left.\mid 1)_{2}|0\rangle, \mid 2\right)_{2}|0\rangle$ and $\left.\mid 1\right)_{2}|1\rangle$ (purple) as well as inactive levels (gray). Other parameters are the same as for the $x$-polarization in Fig. 5.6. The inset shows the validity of the JC-model in the weak coupling limit. 


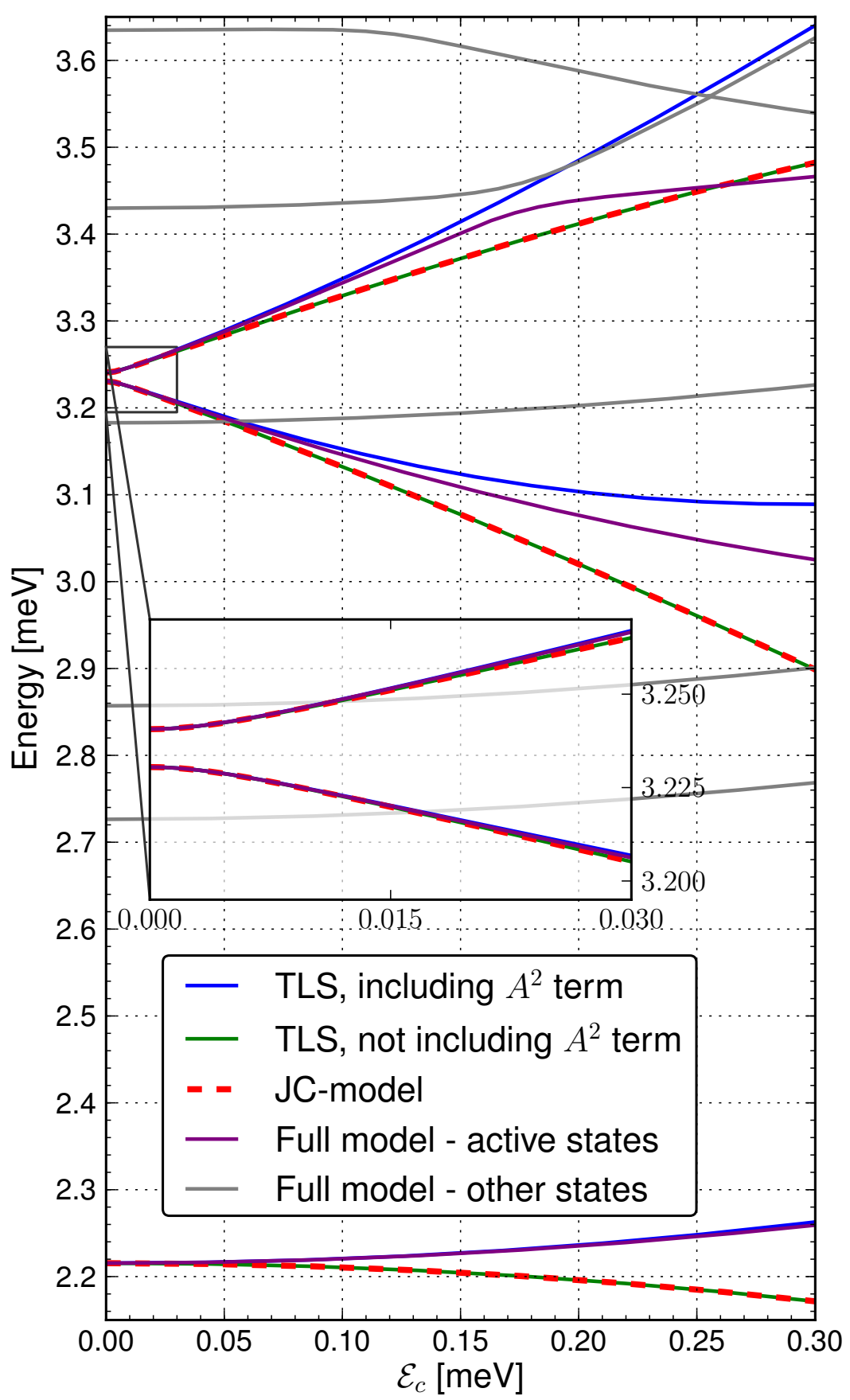

Figure 5.17: Comparison of the many-body energy spectra versus the coupling strength $\mathcal{E}_{\mathrm{c}}$ for the case of two electrons and $y$-polarization. These energy states are obtained by TLS model including the $A^{2}$ term (blue), not including the $A^{2}$ term (green) and the JC-model (red-dashed). The TLS model results are compared with the full numerical calculation for the compared lowest active levels $\left.\mid 1)_{2}|0\rangle, \mid 5\right)_{2}|0\rangle$ and $\left.\mid 1\right)_{2}|1\rangle$ (purple) as well as inactive levels (gray). Other parameters are the same as for the $y$-polarization in Fig. 5.6. The inset shows the validity of the JC-model in the weak coupling limit. 


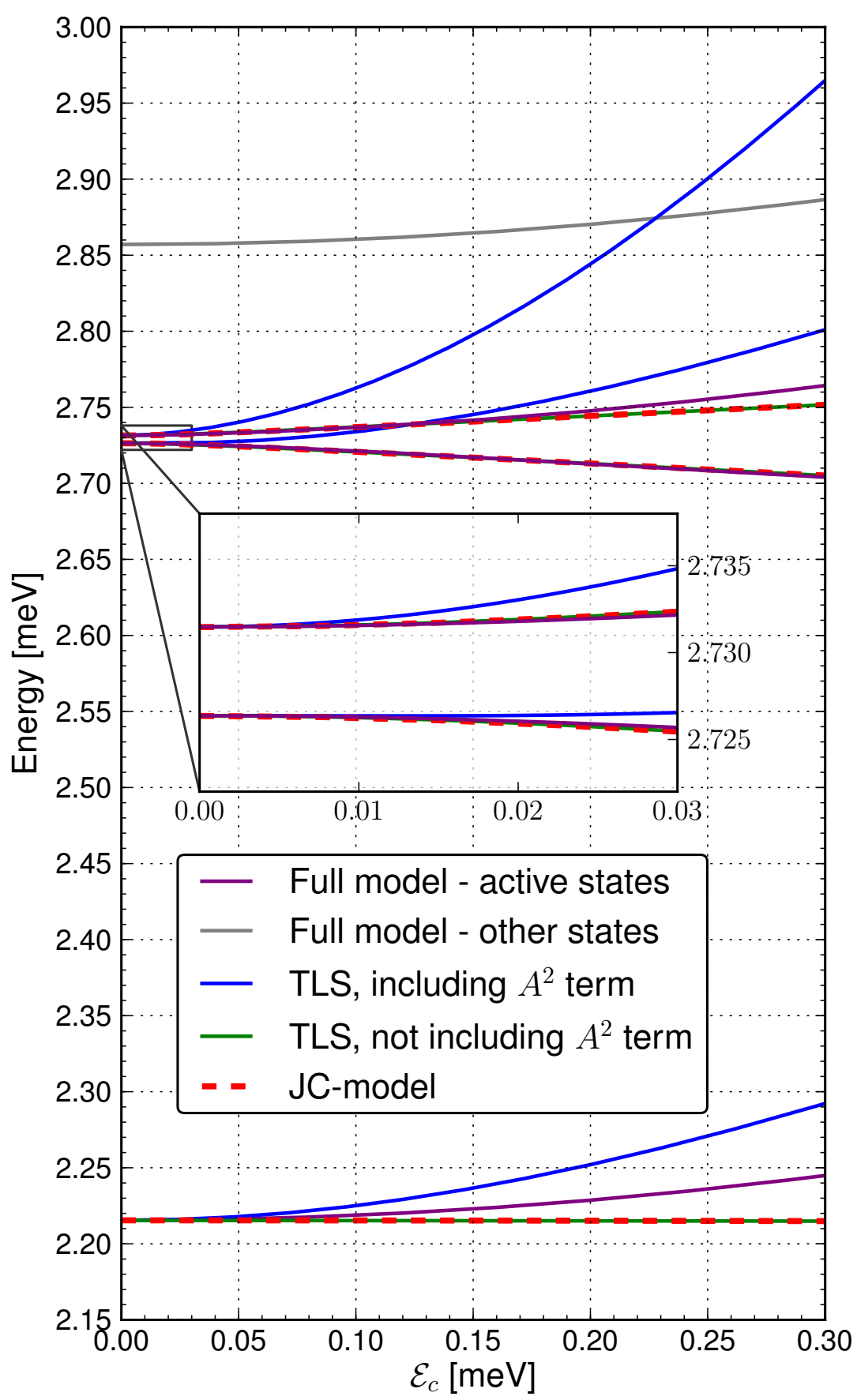

Figure 5.18: Comparison of the many-body energy spectra versus the coupling strength $\mathcal{E}_{\mathrm{c}}$ for the case of two electrons and $y$-polarization. The system is on resonance between the weakly coupled states $\mid 1)_{2}$ and $\left.\mid 2\right)_{2}$ with a DGC strength of $\left|\mathcal{G}_{12}\right|=0.0772, \hbar \omega_{p}=0.516 \mathrm{meV}$ and $\lambda=0.593$. These energy states are obtained by TLS model including the $A^{2}$ term (blue), not including the $A^{2}$ term (green) and the JC approximation without magnetic field (red-dashed). The TLS model results are compared with the full numerical calculation for the compared lowest active levels $\left.\mid 1)_{2}|0\rangle, \mid 2\right)_{2}|0\rangle$ and $\left.\mid 1\right)_{2}|1\rangle$ (purple) as well as inactive levels (gray). 



\section{Convergence tests}

In this section we will investigate how truncation of Fock spaces affect results. I will begin with convergence calculations for one electron and then proceed with many electrons. To get an estimate of the numerical error I define the relative error in the energy of state $\mid \breve{\mu}$ ) as

$$
R_{i j}^{(\mu)} \equiv\left|\frac{E_{i}^{(\mu)}-E_{j}^{(\mu)}}{E_{i}^{(\mu)}}\right|
$$

where $E_{i}^{(\mu)}$ is the energy of state $\left.\mid \breve{\mu}\right)$ and $i$ refers to some accuracy parameter. For example $i$ can be $N_{\text {ses }}, N_{\text {mesT }}$ or $N_{\text {EM }}$. Typically, $j$ is the highest value of the changing parameter that can be used to get results in reasonable time and we vary $i$ to see if the results converge. While I vary the parameters $i$ and $j$, all other accuracy parameters are kept constant. I also define the maximum error of the $N$ lowest states as

$$
R_{i j}^{\max } \equiv \max _{\nu \in[1, N]} R_{i j}^{(\nu)}
$$

The value we choose for $N$ depends on what we intend to use the states for once we are calculating them. For calculating electron transport using the generalized master equation, 64 states are typically used so that is the value I will use [33].

Now that we have a way of approximating the relative error in energy we have to come up with some maximum acceptable error. Ideally we would want at least 16 correct digits, which is the number of digits in double precision floats. However, that would require a basis size beyond what we can handle. I will therefore consider results to have converged when the relative error of the lowest 64 states is less than $10^{-3}$ and less than $10^{-4}$ for the ground state.

Note that in all convergence calculations I use $B=0.1 \mathrm{~T}, \hbar \Omega_{0}=1.0 \mathrm{meV}, \delta=0.01 \Delta_{\alpha \beta}$ and $L_{x}=300 \mathrm{~nm}$, where $\mid \alpha$ ) and $\left.\mid \beta\right)$ are the active electron states. The choice of these two states depends on both polarization and electron number. The range of $\mathcal{E}_{c}$ in which convergence tests are performed is $0 \leq \mathcal{E}_{c} \leq 2 \hbar \omega_{p}$. The grid which the single electron eigenfunctions are saved on and are used for gaussian integration in the Coulomb integral in (3.27) is $160 \times 120$. 


\section{Convergence tests}

\subsection{One electron}

For one electron we only need to investigate convergence with respect to two variables. They are the number of electron states $N_{\text {mesT }}$ (which is equal to $N_{\text {ses }}$ for one electron) and the maximum number of photons $N_{\mathrm{EM}}$. From figures 6.1 and 6.2 we see that convergence with respect to $N_{\text {ses }}$ is much slower than for $N_{\mathrm{EM}}$. We can also see that the results for $N_{\mathrm{EM}}=15$ and $N_{\text {ses }}=200$ are acceptable for $\mathcal{E}_{c}<\hbar \omega_{p}$ for both $x$ and $y$ polarization.
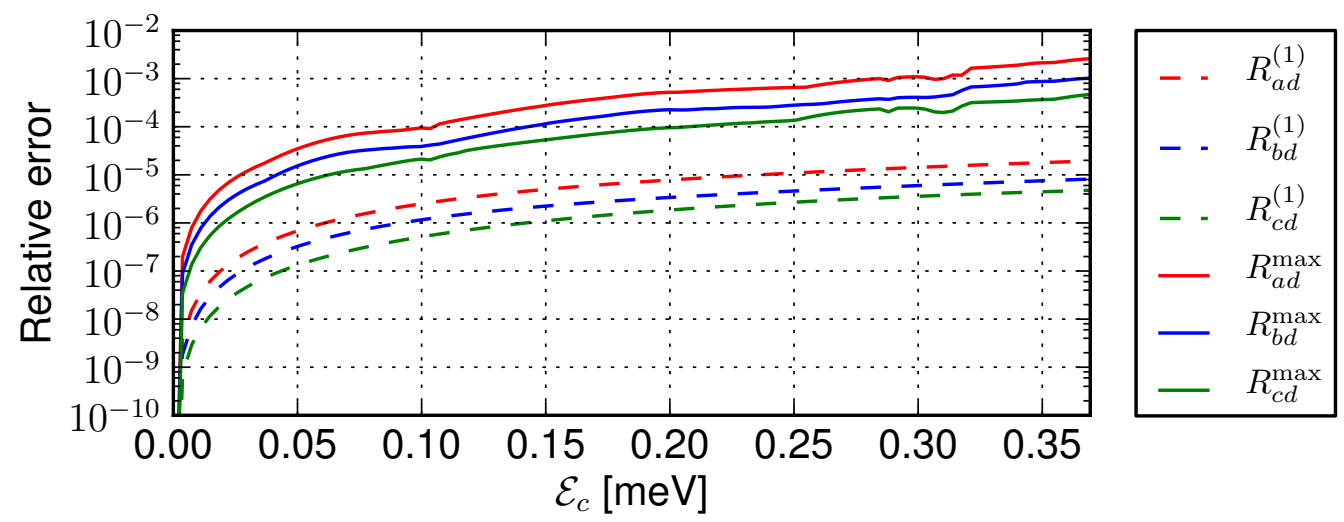

(a) Resonance between the one electron states $\mid 1)_{1}$ and $\left.\mid 2\right)_{1}$ giving $\hbar \omega_{p}=0.185 \mathrm{meV}$. The results are acceptable for $N_{\text {mesT }}=200$ (blue) in the whole range of $\mathcal{E}_{c}$ considered here.

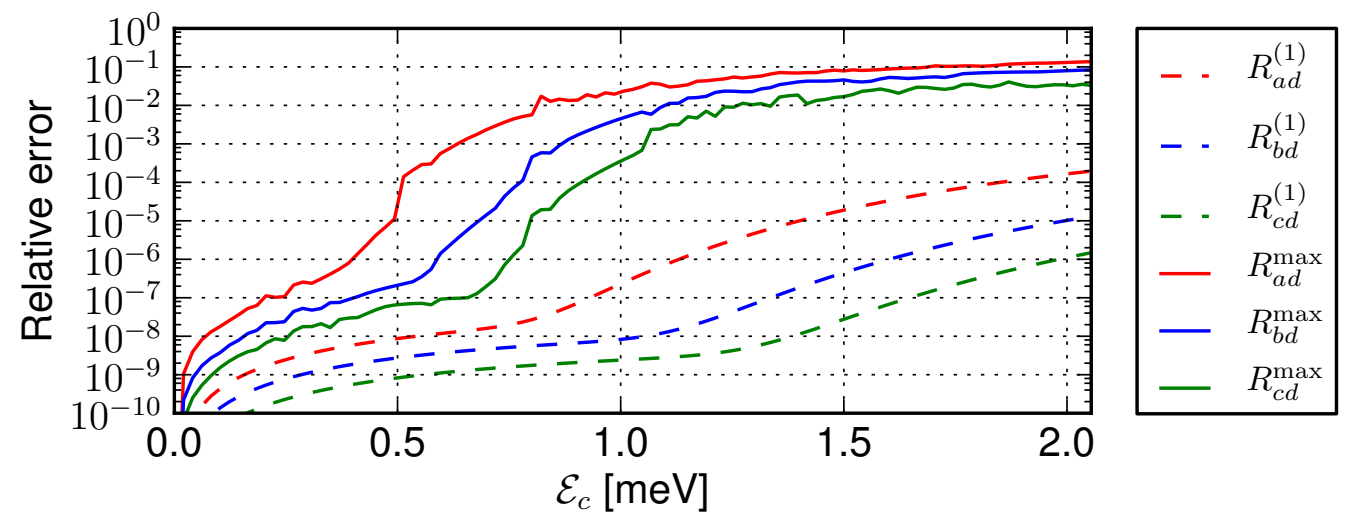

(b) Resonance between the one electron states $\mid 1)_{1}$ and $\left.\mid 5\right)_{1}$ giving $\hbar \omega_{p}=1.03 \mathrm{meV}$. The results are acceptable for $N_{\text {mest }}=200$ (green) up to $\mathcal{E}_{c} \simeq \hbar \omega_{p}$. It's worth noting that the maximum error is up to 5 orders of magnitude higher than the ground state error which is much more than for x-polarization. This indicates that the error rises much faster than for $\mathrm{x}$-polarization when one goes upwards in the energy spectrum. This most likely due to the high amount of energy crossings and anti-crossings for high energy which can be seen on the right panel of Fig. 5.5.

Figure 6.1: Convergence calculations with respect to $N_{\text {mesT }}$ for $x$-polarization (a) and $y$ polarization (b). For this run we have $a=100, b=150, c=200$ and $d=250$ (see equations 6.1 and 6.2 for definition). The maximum number of photons is kept constant at $N_{\mathrm{EM}}=20$. 


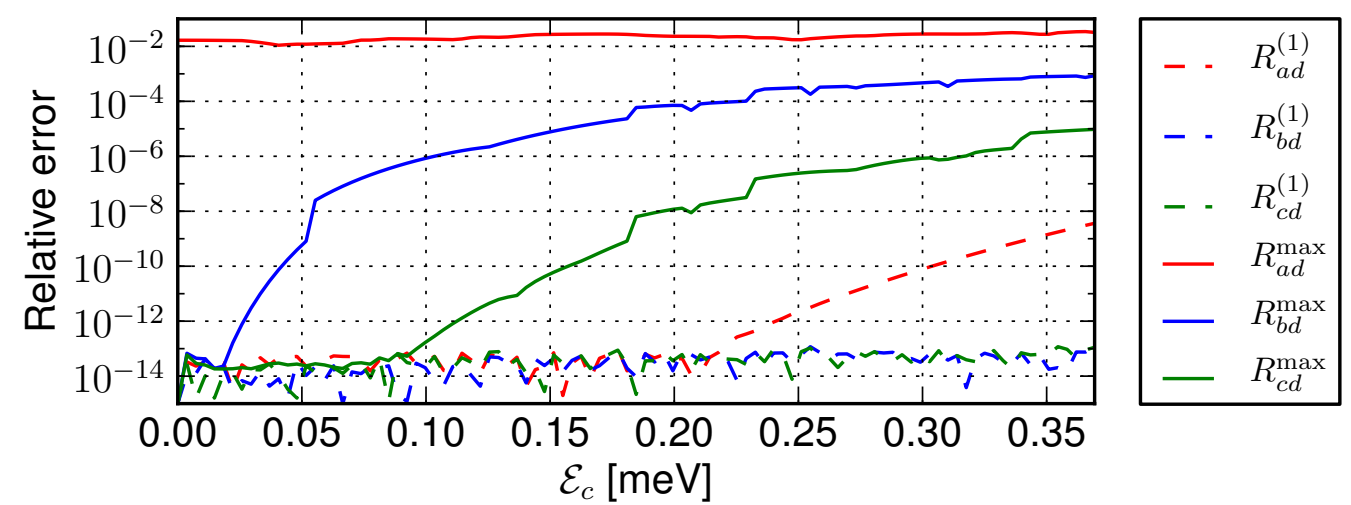

(a) Resonance between the one electron states $\mid 1)_{1}$ and $\left.\mid 2\right)_{1}$ giving $\hbar \omega_{p}=0.185 \mathrm{meV}$. The results are acceptable for $N_{\mathrm{EM}}=15$ (blue) in the whole range of $\mathcal{E}_{c}$ considered here.

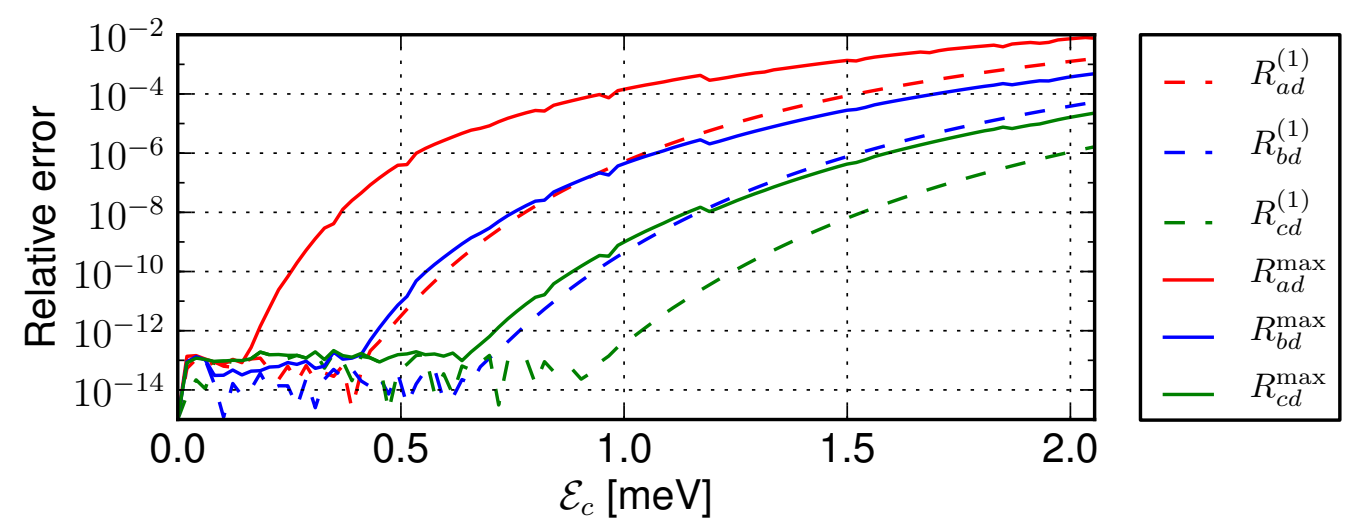

(b) Resonance between the one electron states $\mid 1)_{1}$ and $\left.\mid 5\right)_{1}$ giving $\hbar \omega_{p}=1.03 \mathrm{meV}$. The results are acceptable for $N_{\mathrm{EM}}=15$ (blue) in the whole range of $\mathcal{E}_{c}$ considered here.

Figure 6.2: Convergence calculations with respect to $N_{\mathrm{EM}}$ for $x$-polarization (a) and $y$-polarization (b). For this run we have $a=10, b=15, c=20$ and $d=25$ (see equations 6.1 and 6.2 for definition). The electron state number is kept constant at $N_{\text {mesT }}=200$. We can see that for $N_{E M}=20$, the results are acceptable for the whole range of $\mathcal{E}_{c}$ considered.

\subsection{More than one electron}

For more than one electron we need to investigate convergence with respect to more variables. In addition to $N_{\text {mesT }}$ and $N_{\mathrm{EM}}$ we have $N_{\text {ses }}$ and the size of the grid we use to perform the Coulomb integral in (3.27). These two accuracy parameters don't enter directly into the truncation of the e-EM basis so we don't expect the error to increase much with higher coupling but we still need to obtain some bounds on the error.

Figure 6.3 shows convergence with respect to $N_{\text {mest }}$ which we can see, is much slower for two electrons than one. For $N_{\text {mesT }}=200$ we can get acceptable results up to $\mathcal{E}_{c} / \hbar \omega_{p} \simeq 0.5$ for $x$-polarization and $\mathcal{E}_{c} / \hbar \omega_{p} \simeq 0.25$ for $y$-polarization. The convergence with respect to 


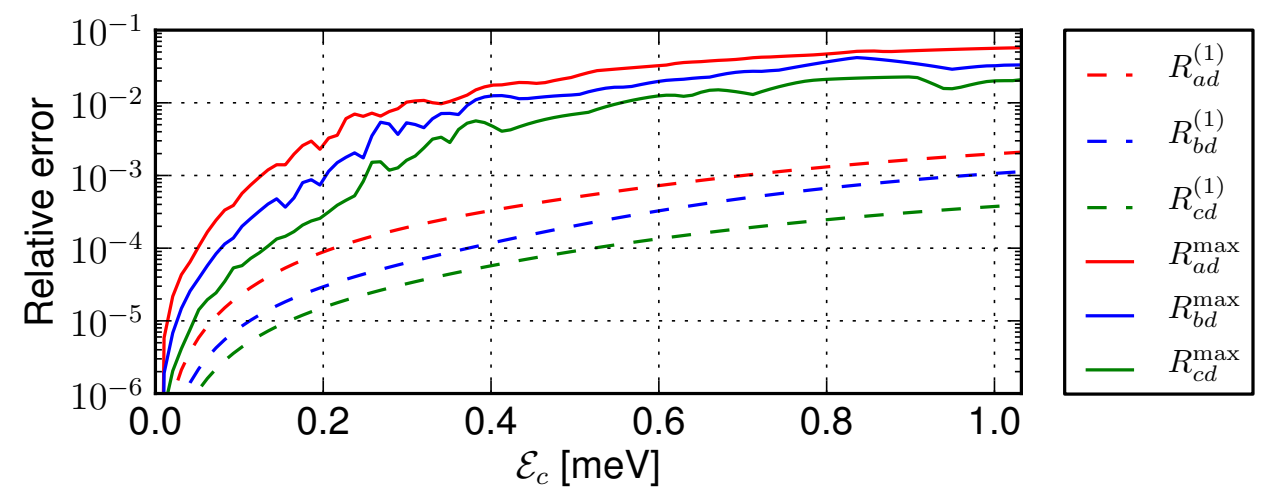

(a) Resonance between the two electron states $\mid 1)$ and $\mid 2)$ giving $\hbar \omega_{p}=0.516 \mathrm{meV}$. The results are acceptable for $N_{\text {mesT }}=200$ (green) up to $\mathcal{E}_{c} \simeq \hbar \omega_{p} / 2$.

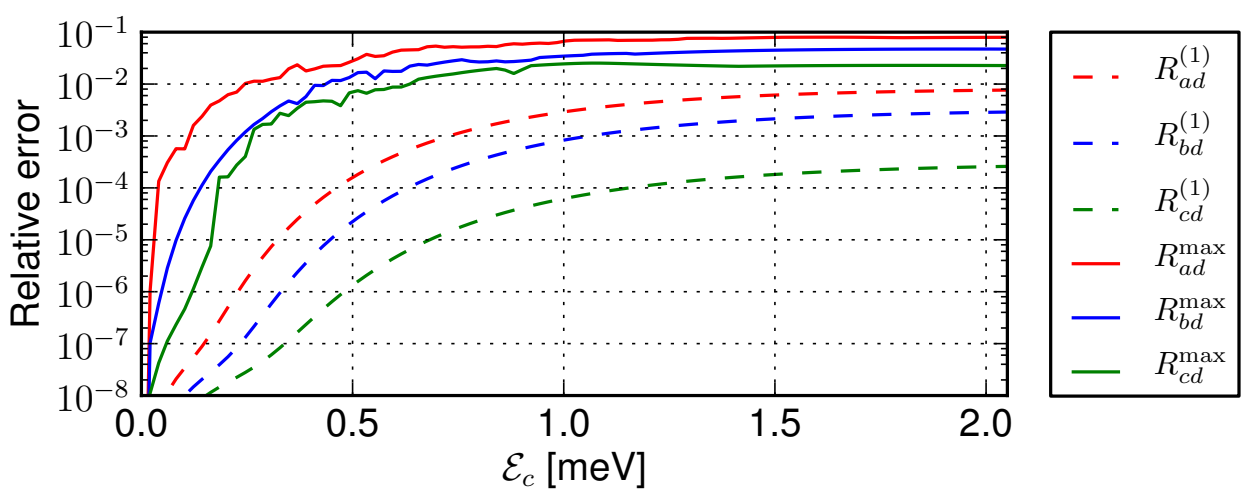

(b) Resonance between the two electron states $\mid 1$ ) and $\mid 5)$ giving $\hbar \omega_{p}=1.025 \mathrm{meV}$. The results are acceptable for $N_{\text {mesT }}=200$ The results are acceptable for $N_{\text {mesT }}=200$ (green) up to $\mathcal{E}_{c} \simeq \hbar \omega_{p} / 4$.

Figure 6.3: Convergence calculations for two electrons with respect to $N_{\text {mesT }}$ for $x$-polarization (a) and $y$-polarization (b). For this run we have $a=100, b=150, c=200$ and $d=250$ (see equations 6.1 and 6.2 for definition). Other accuracy parameters are $N_{\text {ses }}=50$ and $N_{\mathrm{EM}}=20$.

$N_{\text {EM }}$ was found to be almost identical with the one electron result in 6.1 so we conclude that we can safely put $N_{\mathrm{EM}}=20$ and not worry about it since the error due to truncation of the electron Fock space is much greater. Fig. 6.4 shows convergence with respect to $N_{\text {ses }}$ and the grid size for $y$-polarization, which is considerably faster than for $N_{\text {mest }}$. At this point it is clear that the truncation of the electron Fock space is the main cause of numerical error so in the remainder of this section I will concentrate on convergence with respect to $N_{\text {mesT }}$ and ignore other accuracy parameters.

Figure 6.5 shows convergence calculations with respect to $N_{\text {mesT }}$ for three electrons. We can see that the convergence is a bit slower than for two electrons. For $N_{\text {mest }}=200$, $N_{\mathrm{EM}}=20$ and $N_{\mathrm{ses}}=30$, we can get acceptable results up to $\mathcal{E}_{c} / \hbar \omega_{p}=0.4$ for $x$ polarization and $\mathcal{E}_{c} / \hbar \omega_{p}=0.2$ for $y$-polarization. I expect this trend of slower convergence with increasing electrons number to continue. However, I will not continue convergence calculations beyond three electrons in this thesis. 


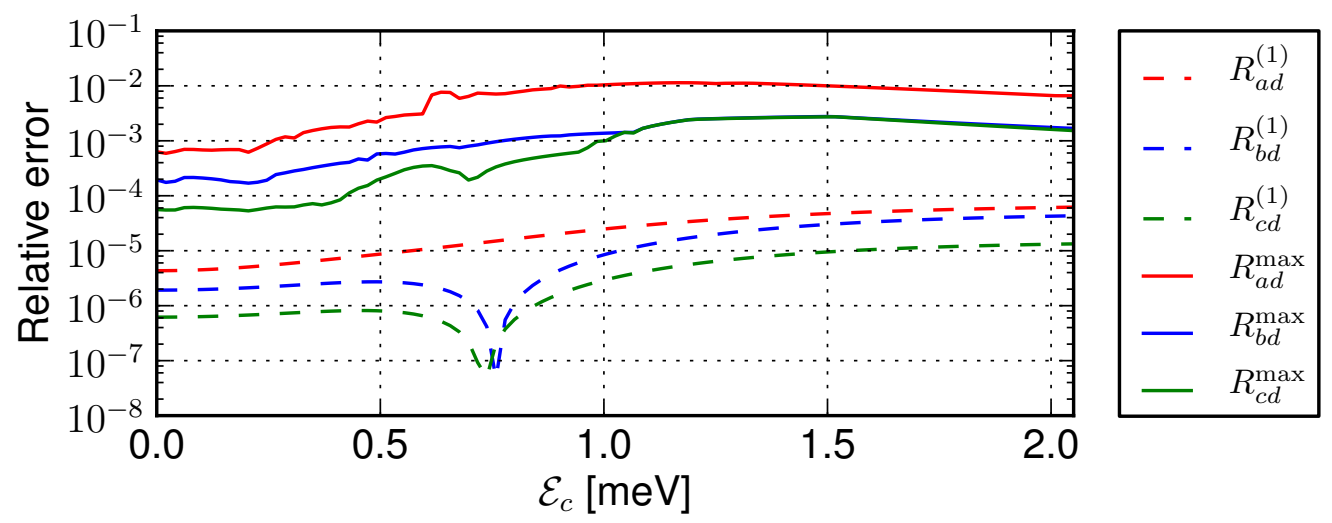

(a) For this run we have $a=30, b=40, c=50$ and $d=60$ (see equations 6.1 and 6.2 for definition). The results are acceptable for $N_{\text {ses }}=50$ (green) up to $\mathcal{E}_{c} \simeq \hbar \omega_{p}$.

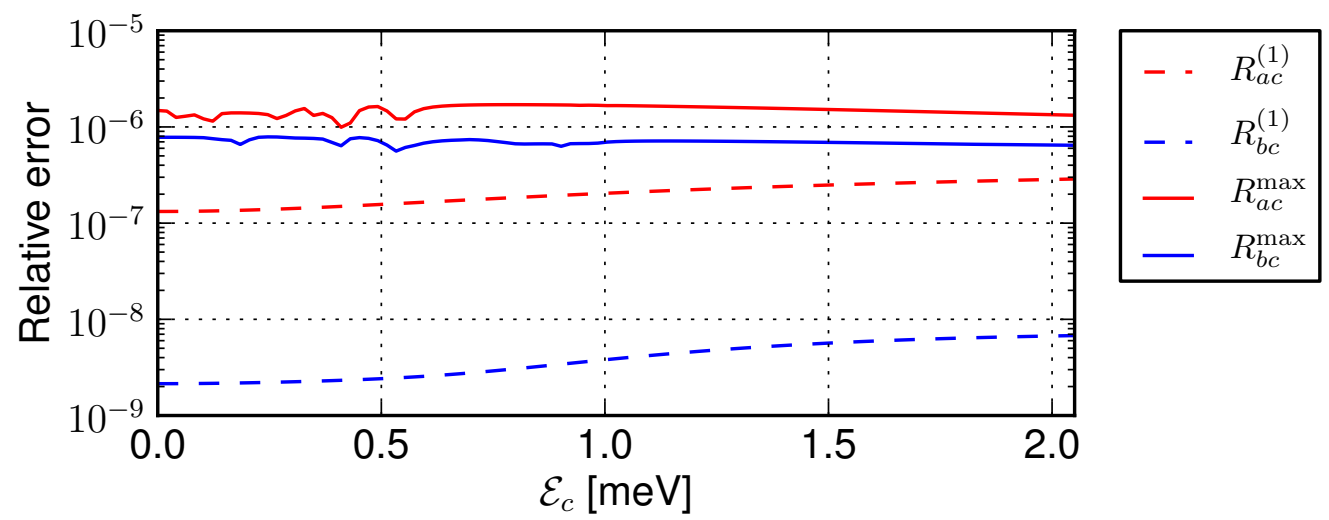

(b) For this run, $a$ is a $80 \times 60$ grid, $b$ is $160 \times 120$ and $c$ is $220 \times 160$ (see equations 6.1 and 6.2 for definition). From the data we can see that a 80x60 grid is sufficient to get acceptable accuracy. However, I will use the $160 \times 120$ grid in calculations since it requires minimal additional computation time.

Figure 6.4: Convergence calculations for two electrons with respect to $N_{\text {ses }}$ (a) and grid size (b). In both cases, $y$-polarization is used and we have resonance between the two electron states $\mid 1)$ and $\mid 5)$ giving $\hbar \omega_{p}=1.025 \mathrm{meV}$. The corresponding results for $x$-polarization are almost identical and will be omitted.

So far we have concentrated on a system that is on resonance, that is the quantized EM field frequency matches an energy gap between two states $\mid \mu)$ and $\mid \nu)$ with relatively strong DGC (large $\left|\mathcal{G}_{\alpha \beta}\right|$ ). To see if convergence differs for a system off resonance I performed convergence calculations for a two electron system which is off resonance (see Fig. 6.6). By comparison with Fig. 6.3 we see that the convergence with respect to $N_{\text {mesT }}$ is considerably faster for a system that is off resonance, especially for $y$-polarization.

To conclude this section, I will show that the $A^{2}$ term has a drastic effect on convergence. Figure 6.7 shows convergence calculations with respect to $N_{\text {mesT }}$ without the $A^{2}$ term. As can be seen from the figure, the results blow up at around $\mathcal{E}_{c} \simeq 0.75 \hbar \omega_{p} \simeq 0.4 \mathrm{meV}$. Comparing the results with Fig. 6.3a, in which the $A^{2}$ term is included. We can see that the convergence is much better in the latter mentioned figure. 


\section{Convergence tests}

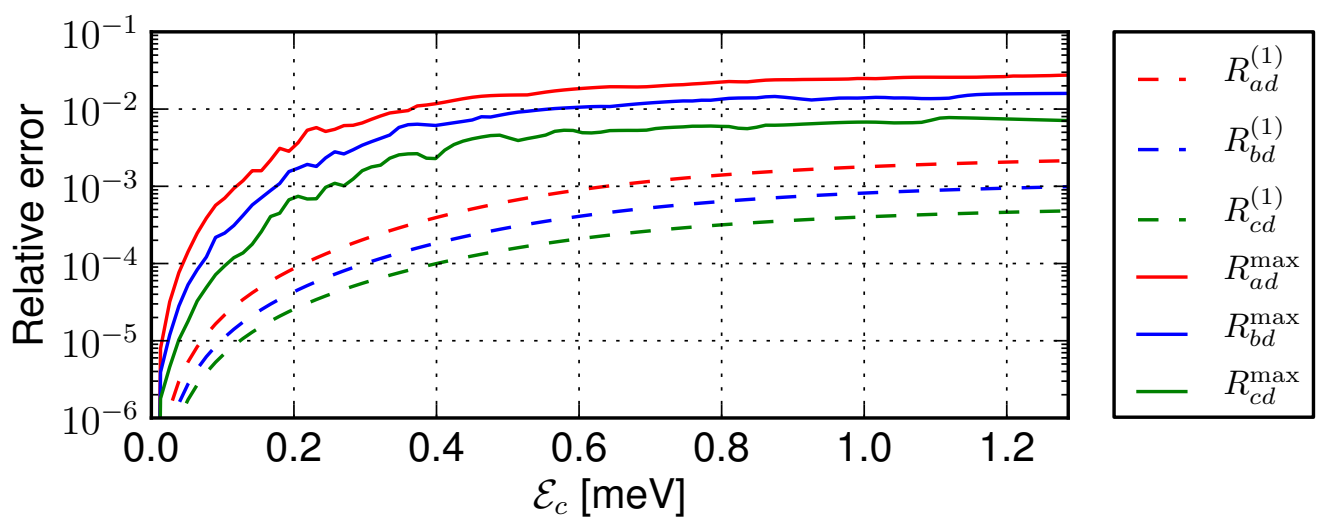

(a) Resonance between the three electron states $\mid 1$ ) and $\mid 3)$ giving $\hbar \omega_{p}=0.643 \mathrm{meV}$. The results are acceptable for $N_{\text {mesT }}=200$ (green) up to $\mathcal{E}_{c} \simeq 0.4 \hbar \omega_{p}$.

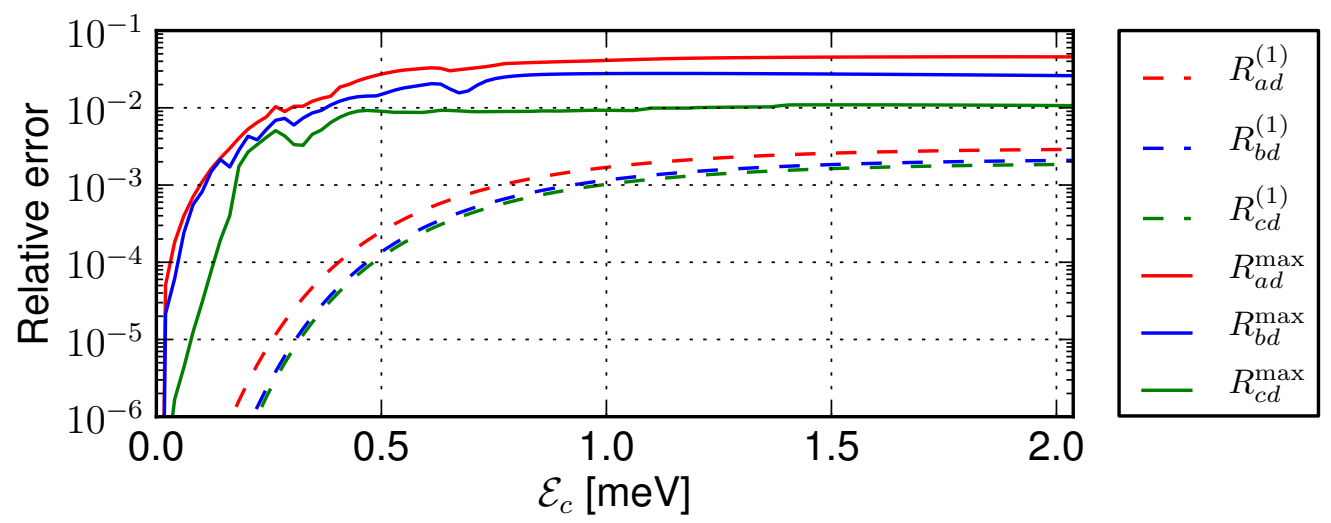

(b) Resonance between the three electron states $\mid 1$ ) and $\mid 5)$ giving $\hbar \omega_{p}=1.02 \mathrm{meV}$. The results are acceptable for $N_{\text {mesT }}=200$ (green) up to $\mathcal{E}_{c} \simeq 0.2 \hbar \omega_{p}$.

Figure 6.5: Convergence calculations for three electrons with respect to $N_{\text {mesT }}$ for $x$-polarization (b) and $y$-polarization (b). For this run we have $a=100, b=150, c=200$ and $d=250$ (see equations 6.1 and 6.2 for definition). Other accuracy parameters are $N_{\mathrm{ses}}=30$ and $N_{\mathrm{EM}}=20$. 


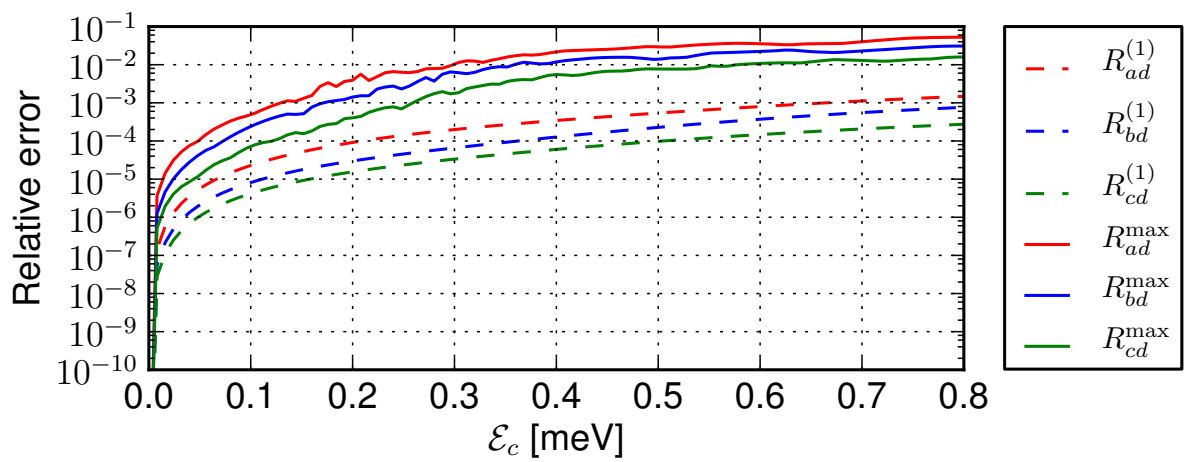

(a) Results are acceptable for $N_{\text {mesT }}=200$ (green) up to $\mathcal{E}_{c} \simeq 0.65 \hbar \omega_{p}$.

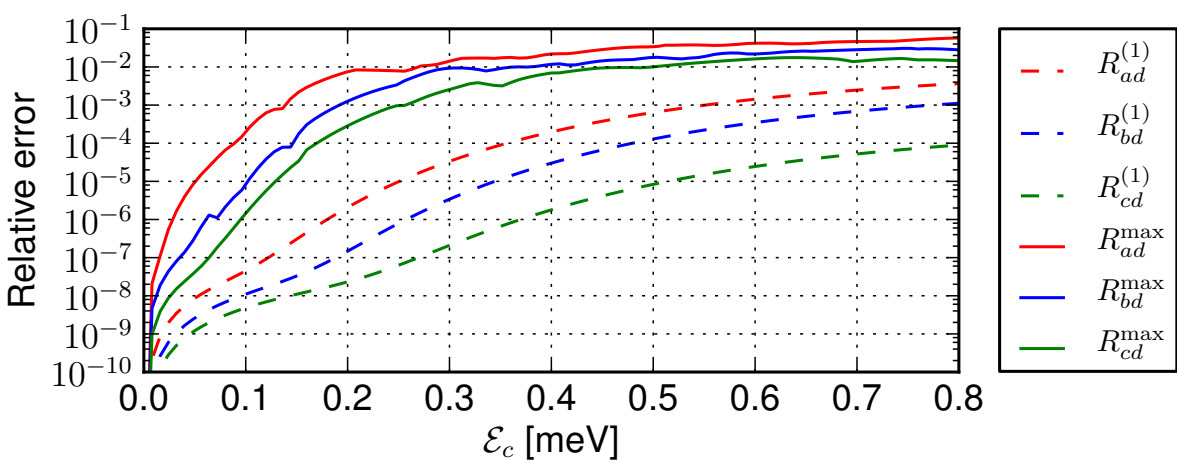

(b) Results are acceptable for $N_{\text {mesT }}=200$ (green) up to $\mathcal{E}_{c} \simeq 0.65 \hbar \omega_{p}$.

Figure 6.6: Convergence calculations for two electrons with respect to $N_{\text {mesT }}$ for $x$-polarization (b) and $y$-polarization (b). The system is off resonance with $\hbar \omega_{p}=0.4 \mathrm{meV}$ for both polarizations. For this run we have $a=100, b=150, c=200$ and $d=250$ (see equations 6.1 and 6.2 for definition). Other accuracy parameters are $N_{\text {ses }}=50$ and $N_{\mathrm{EM}}=20$.

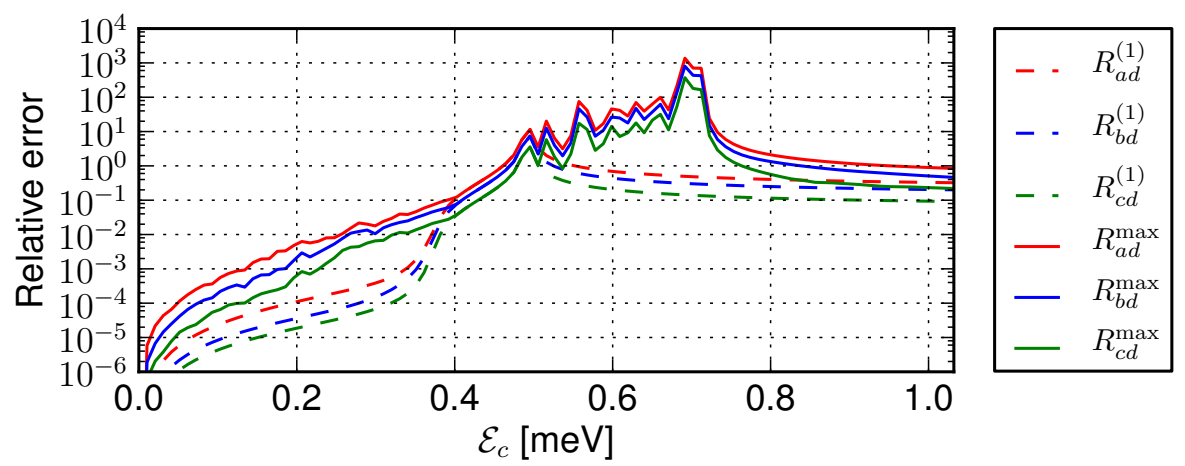

Figure 6.7: Convergence calculations with respect to $N_{\text {mesT }}$ for $x$-polarization, ignoring the $A^{2}$ term in the e-EM interaction Hamiltonian. The system is on resonance between the two electron states $\mid 1)_{2}$ and $\left.\mid 2\right)_{2}$ giving $\hbar \omega_{p}=0.516$. For this run we have $a=100, b=150, c=200$ and $d=250$ (see equations 6.1 and 6.2 for definition). The results start to blow up at around $\mathcal{E}_{c} \simeq 0.4$. At around $\mathcal{E}_{c} \simeq 0.7$, the error is a 1000 times larger than the calculated value of the energy. 



\section{Conclusion}

We have performed a numerical calculation of a microscopic model describing a hybrid structure consisting of an electronic nanostructure embedded in a cavity resonator. We have demonstrated strong coupling features of Coulomb interacting electrons and photons in an external magnetic field. The two-dimensional electron gas in the nanostructure is parabolically confined in the $y$-direction and hard-wall confined in the $x$ direction that is embedded in a rectangular photon cavity with a TE-mode electromagnetic field that may be either $x$ - or $y$-polarized.

We have established that the diamagnetic $A^{2}$ term in the e-EM interaction Hamiltonian provides a blue-shift correction to the energy spectrum. However, including higher manybody states beyond a two-level approximation results in a smaller red-shift correction. This implies that the two lowest levels become more stable when the higher energy levels are included in the electron-photon coupled system. When the $A^{2}$ term is not included in calculations, energy spectra take a steep dive downwards in energy when the e-EM coupling strength is comparable in magnitude to the photon energy. This behavior has no physical significance since the results are highly divergent in this strong coupling regime without the diamagnetic $A^{2}$ term. Including the $A^{2}$ term eliminates this behavior and drastically improves numerical convergence.

A widely employed two-level system approximation has been reexamined comparing to results of our full numerical calculation model for one and two electrons. Our numerical results demonstrate that the two-level system approximation and the Jaynes-Cummings model remain valid in the weak electron-photon coupling regime. However qualitative difference of the energy spectrum between the TLS models and the full numerical calculation is found for strong e-EM coupling. The TLS approximations all fail around energy anti-crossings, while fairing much better around energy crossings. From these results, it is apparent that QED modeling of a circuit element on the nanoscale in the ultrastrong coupling regime requires approximations beyond the JC-model or more general two level models.

Two level systems such as the JC-model include no direct information about the electron charge distribution of the system. However, with our model, we were able to calculate electron charge densities for the lower eigenstates. We observed that the charge density of the ground state changes by a very small amount, even in the ulta strong coupling regime when the e-EM coupling strength is double that of the cavity photon energy. When 
the e-EM coupling strength is tuned up, charge densities of excited states only change considerably around energy anti-crossings. When the the charge density does change, it does so rapidly in a small interval of e-EM coupling and does not change considerably again until the next anti-crossing.

In summary, we have presented a model adequate for accurate numerical calculation for the electron-photon coupled energy spectrum that is essential and was utilized to explore time-dependent transport of electrons through a photon cavity [33]. 


\section{A. Detailed calculations of matrix elements}

\section{A.1. Evaluating $I_{\mathrm{x}}^{\mathrm{nn}^{\prime}}$}

We need to evaluate $I_{x}^{n n^{\prime}}$ for 4 cases.

- Both $n$ and $n^{\prime}$ even $\left(I_{x}^{e e}\right)$.

- Both $n$ and $n^{\prime}$ odd $\left(I_{x}^{o o}\right)$.

- $n$ even and $n^{\prime}$ odd $\left(I_{x}^{e o}\right)$.

- $n$ odd and $n^{\prime}$ even $\left(I_{x}^{o e}\right)$.

I will start with $I_{x}^{e e}$ and work my way down.

$$
I_{x}^{e e}=2 \frac{n^{\prime} \pi}{L_{x}} \int_{-L_{x} / 2}^{L_{x} / 2} \sin \left(\frac{n \pi}{L_{x}} x\right) \cos \left(\frac{n^{\prime} \pi}{L_{x}} x\right) \mathrm{d} x=0
$$

because $\cos (a x) \sin (b x)$ is an odd function, which also means that

$$
I_{x}^{o o}=-2 \frac{n^{\prime} \pi}{L_{x}} \int_{-L_{x} / 2}^{L_{x} / 2} \cos \left(\frac{n \pi}{L_{x}} x\right) \sin \left(\frac{n^{\prime} \pi}{L_{x}} x\right) \mathrm{d} x=0 .
$$

Now the even-odd case.

$$
I_{x}^{e o}=-2 \frac{n^{\prime} \pi}{L_{x}} \int_{-L_{x} / 2}^{L_{x} / 2} \sin \left(\frac{n \pi}{L_{x}} x\right) \sin \left(\frac{n^{\prime} \pi}{L_{x}} x\right) \mathrm{d} x .
$$




\section{A. Detailed calculations of matrix elements}

Put $z=x / L_{x}$ and note that $\sin (a x) \sin (b x)$ is an even function so we can change the integration limits

$$
\begin{gathered}
I_{x}^{e o}=-4 n^{\prime} \pi \int_{0}^{1 / 2} \sin (n \pi z) \sin \left(n^{\prime} \pi z\right) \mathrm{d} z \\
=2 n^{\prime} \pi \int_{0}^{1 / 2}\left[\cos \left(\left(n+n^{\prime}\right) \pi z\right)-\cos \left(\left(n-n^{\prime}\right) \pi z\right)\right] \mathrm{d} z
\end{gathered}
$$

where I've used that $\sin (a) \sin (b)=-\frac{1}{2}[\cos (a+b)-\cos (a-b)]$. Performing the integration in (A.4) we obtain

$$
I_{x}^{e o}=2 n^{\prime}\left[\frac{\sin \left(\left(n+n^{\prime}\right) \pi / 2\right)}{n+n^{\prime}}-\frac{\sin \left(\left(n-n^{\prime}\right) \pi / 2\right)}{n-n^{\prime}}\right] .
$$

This can be simplified to

$$
I_{x}^{e o}=\frac{-4 n n^{\prime}}{n^{2}-n^{\prime 2}}(-1)^{\left(n+n^{\prime}+1\right) / 2}
$$

Now the odd-even case.

$$
I_{x}^{o e}=2 \frac{n^{\prime} \pi}{L_{x}} \int_{-L_{x} / 2}^{L_{x} / 2} \cos \left(\frac{n \pi}{L_{x}} x\right) \cos \left(\frac{n^{\prime} \pi}{L_{x}} x\right) \mathrm{d} x .
$$

Put $z=x / L_{x}$ and note that $\cos (a x) \cos (b x)$ is an even function so we can change the integration limits

$$
\begin{gathered}
I_{x}^{o e}=4 n^{\prime} \pi \int_{0}^{1 / 2} \cos (n \pi z) \cos \left(n^{\prime} \pi z\right) \mathrm{d} z \\
=2 n^{\prime} \pi \int_{0}^{1 / 2}\left[\cos \left(\left(n+n^{\prime}\right) \pi z\right)+\cos \left(\left(n-n^{\prime}\right) \pi z\right)\right] \mathrm{d} z
\end{gathered}
$$

where I've used that $\cos (a) \cos (b)=\frac{1}{2}[\cos (a+b)+\cos (a-b)]$. Performing the integration in (A.8) we obtain

$$
I_{x}^{o e}=2 n^{\prime}\left[\frac{\sin \left(\left(n+n^{\prime}\right) \pi / 2\right)}{n+n^{\prime}}+\frac{\sin \left(\left(n-n^{\prime}\right) \pi / 2\right)}{n-n^{\prime}}\right] .
$$

This can be simplified to

$$
I_{x}^{o e}=\frac{-4 n n^{\prime}}{n^{2}-n^{\prime 2}}(-1)^{\left(n+n^{\prime}+1\right) / 2}
$$

which is the same result we got in (A.6). 


\section{A.2. Evaluating $I_{\mathrm{y}}^{\mathrm{mm}^{\prime}}$}

The $I_{y}^{m m^{\prime}}$ integral is a bit easier to evaluate than $I_{x}^{n n^{\prime}}$.

$$
\begin{gathered}
I_{y}^{m m^{\prime}}=\frac{1}{a_{w}} \int_{-\infty}^{\infty} \varphi_{m}(y) y \varphi_{m^{\prime}}(y) \mathrm{d} y \\
=\frac{1}{\sqrt{2^{m} \sqrt{\pi} m !}} \frac{1}{\sqrt{2^{m^{\prime}} \sqrt{\pi} m^{\prime} !}} \int_{-\infty}^{\infty} e^{-z^{2}} z H_{m}(z) H_{m^{\prime}}(z) \mathrm{d} z
\end{gathered}
$$

We now use the recursion relation

$$
z H_{m}(z)=\frac{1}{2} H_{n+1}(z)+n H_{n-1}(z)
$$

and (A.12) becomes

$$
\begin{gathered}
\frac{1}{\sqrt{2^{m} \sqrt{\pi} m !}} \frac{1}{\sqrt{2^{m^{\prime}} \sqrt{\pi} m^{\prime} !}} \int_{-\infty}^{\infty} e^{-z^{2}} H_{m^{\prime}}\left\{\frac{1}{2} H_{m+1}(z)+m H_{m-1}(z)\right\} \mathrm{d} z \\
=\sqrt{\frac{m^{\prime}+1}{2}} \delta_{m, m^{\prime}+1}+\sqrt{\frac{m^{\prime}}{2}} \delta_{m, m^{\prime}-1}
\end{gathered}
$$

\section{A.3. Evaluating $G_{\mathbf{y}}^{\mathrm{mm}^{\prime}}$}

$$
G_{y}^{m m^{\prime}}=\frac{1}{\sqrt{2^{m+m^{\prime}} \pi m ! m^{\prime} !}} \int_{-\infty}^{\infty} H_{m}(\xi) H_{m^{\prime}}(\xi) e^{-\xi^{2}} e^{-\alpha_{y}^{2}\left(\xi-\xi_{0}\right)^{2}} \mathrm{~d} \xi .
$$

We fill the square in (A.16) and shift the integration variable by $\xi_{0}$ which has no effect on the limits and get

$$
G_{y}^{m m^{\prime}}=\frac{e^{-\frac{\alpha_{y}^{2} \xi_{0}^{2}}{\alpha_{y}^{2}+1}}}{\sqrt{2^{m+m^{\prime}} \pi m ! m^{\prime} !}} \int_{-\infty}^{\infty} H_{m}\left(\xi+\xi_{0}\right) H_{m^{\prime}}\left(\xi+\xi_{0}\right) e^{-\left(\alpha_{y}^{2}+1\right) \xi^{2}} \mathrm{~d} \xi
$$




\section{A. Detailed calculations of matrix elements}

Now use that

$$
H_{n}(x+y)=\sum_{k=0}^{n}\left(\begin{array}{l}
n \\
k
\end{array}\right) H_{k}(x)(2 y)^{(n-k)}
$$

and (A.18) becomes

$$
\begin{aligned}
\frac{e^{-\frac{\alpha_{y}^{2} \xi_{0}^{2}}{\alpha_{y}^{2}+1}}\left(\frac{2 \alpha_{y}^{2} \xi_{0}}{\alpha_{y}^{2}+1}\right)^{m+m^{\prime}}}{\sqrt{2^{m+m^{\prime}} \pi m ! m^{\prime} !}} \sum_{k=0}^{m} \sum_{\ell=0}^{m^{\prime}}\left(\begin{array}{c}
m \\
k
\end{array}\right)\left(\begin{array}{c}
m^{\prime} \\
\ell
\end{array}\right)\left(\frac{\alpha_{y}^{2}+1}{2 \alpha_{y}^{2} \xi_{0}}\right)^{k+\ell} \\
\times \int_{-\infty}^{\infty} H_{m}(\xi) H_{m^{\prime}}(\xi) e^{-2\left(\sqrt{\frac{\alpha_{y}^{2}+1}{2}}\right)^{2} \xi^{2}} \mathrm{~d} \xi
\end{aligned}
$$

Finally we use [34, eq. 7.374.5], which states

$$
\begin{aligned}
& \int_{-\infty}^{\infty} H_{m}(\xi) H_{n}(\xi) e^{-2 a^{2} \xi^{2}} \mathrm{~d} \xi=2^{(m+n-1) / 2} a^{-m-n-1}\left(1-2 a^{2}\right)^{(m+n) / 2} \\
& \quad \times \Gamma\left(\frac{m+n+1}{2}\right){ }_{2} F_{1}\left(-n,-m ; \frac{1-m-n}{2} ; \frac{a^{2}}{2 a^{2}-1}\right) .
\end{aligned}
$$

If we use (A.19) and (A.20) with $a=\sqrt{\frac{\alpha_{y}^{2}+1}{2}}$ we get (2.12). This result does not work if $\xi_{0}=0$ so for that case we need to go back to (A.16) and put $\xi_{0}=0$ and get

$$
G_{y}^{m m^{\prime}}(\xi=0)=\frac{1}{\sqrt{2^{m+m^{\prime}} \pi m ! m^{\prime} !}} \int_{-\infty}^{\infty} H_{m}(\xi) H_{m^{\prime}}(\xi) e^{-2\left(\sqrt{\frac{\alpha_{y}^{2}+1}{2}}\right) \xi^{2}} \mathrm{~d} \xi .
$$

We again use (A.20) in (A.21) and get (2.14).

\section{A.4. Evaluating $g_{i j}$}

Our starting point is Eq. (4.16);

$$
g_{i j}=\left\langle\psi_{i}|g| \psi_{j}\right\rangle \equiv\left\langle\psi_{i}\left|\frac{q}{2 m}\left(\boldsymbol{\pi} \cdot \mathbf{A}_{\mathrm{EM}}+\mathbf{A}_{\mathrm{EM}} \cdot \boldsymbol{\pi}\right)\right| \psi_{j}\right\rangle
$$

Because $\mathbf{A}_{\mathrm{EM}}$ was approximated as constant on the wire we have $\left[\mathbf{A}_{\mathrm{EM}}, \boldsymbol{\pi}\right] \simeq \mathbf{0}$, so

$$
g_{i j} \simeq\left\langle\psi_{i}\left|\frac{q}{m}\left(\mathbf{A}_{\mathrm{EM}} \cdot \boldsymbol{\pi}\right)\right| \psi_{j}\right\rangle .
$$


Plugging in $\pi_{x}=p_{x}+q A_{x}=-i \hbar \partial_{x}-q B y$ and $\pi_{y}=p_{y}+q A_{y}=-i \hbar \partial_{y}$ we obtain

$$
\begin{array}{r}
g_{i j} \simeq \frac{q A_{\mathrm{EM}}}{m}\left\langle\psi_{i}\left|e_{x}\left(-i \hbar \partial_{x}-q B y\right)+e_{y}\left(-i \hbar \partial_{y}\right)\right| \psi_{j}\right\rangle \\
=\mathcal{E}_{c}\left[-i e_{x} \frac{a_{w}}{L_{x}}\left\langle\psi_{i}\left|L_{x} \partial_{x}\right| \psi_{j}\right\rangle-i e_{y}\left\langle\psi_{i}\left|a_{w} \partial_{y}\right| \psi_{j}\right\rangle\right. \\
\left.-e_{x} \frac{\omega_{c}}{\Omega_{w}}\left\langle\psi_{i}\left|y / a_{w}\right| \psi_{j}\right\rangle\right] .
\end{array}
$$

I will solve this integral in the original one electron basis $\left\{\left|m_{i}, n_{i}\right\rangle\right\}$ defined in 2.2. Then I transform the results into the $\left\{\left|\psi_{i}\right\rangle\right\}$ basis using the unitary transform $U$ defined in 2.18. This means we need to evaluate

$$
\begin{array}{r}
\left\langle m_{i}, n_{i}|g| m_{j}, n_{j}\right\rangle=\mathcal{E}_{c}\left\langle n_{i}, m_{i}\right|\left\{-i e_{x} \frac{a_{w}}{L_{x}}\left(L_{x} \partial_{x}\right)-i e_{y}\left(a_{w} \partial_{y}\right)\right. \\
\left.-e_{x} \frac{\omega_{c}}{\Omega_{w}}\left(y / a_{w}\right)\right\}\left|m_{j}, n_{j}\right\rangle \\
=\mathcal{E}_{c}\left\{-i e_{x} \frac{a_{w}}{L_{x}}\left\langle n_{i}\left|L_{x} \partial_{x}\right| n_{j}\right\rangle \delta_{m_{i}, m_{j}}-i e_{y}\left\langle m_{i}\left|a_{w} \partial_{y}\right| m_{j}\right\rangle \delta_{n_{i}, n_{j}}\right. \\
\left.-e_{x} \frac{\omega_{c}}{\Omega_{w}}\left\langle m_{i}\left|\frac{y}{a_{w}}\right| m_{j}\right\rangle \delta_{n_{i}, n_{j}}\right\} .
\end{array}
$$

where $\left\langle n_{i}\left|L_{x} \partial_{x}\right| n_{j}\right\rangle$ and $\left\langle m_{i}\left|\frac{y}{a_{w}}\right| m_{j}\right\rangle$ we have already been calculated in A.1 and A.2. Using the recursion relation for Hermite functions (eigenfunctions of the Harmonic oscillator) we calculate the remaining integral

$$
\begin{array}{r}
\left\langle m_{i}\left|a_{w} \partial_{y}\right| m_{j}\right\rangle=\left\langle m_{i}\right| \\
\left(\sqrt{\frac{m_{j}}{2}}\left|m_{j}-1\right\rangle-\sqrt{\frac{m_{j}+1}{2}}\left|m_{j}+1\right\rangle\right) \\
=\sqrt{\frac{m_{j}}{2}} \delta_{m_{i}, m_{j}+1}-\sqrt{\frac{m_{j}+1}{2}} \delta_{m_{i}, m_{j}+1} .
\end{array}
$$

Putting everything together we obtain

$$
\begin{gathered}
\left\langle m_{i}, n_{i}|g| m_{j}, n_{j}\right\rangle=\mathcal{E}_{c}\left\{-e_{x} \frac{\omega_{c}}{\Omega_{w}}\left[\sqrt{\frac{m_{j}+1}{2}} \delta_{m_{i}, m_{j}+1}+\sqrt{\frac{m_{j}}{2}} \delta_{m_{i}, m_{j}-1}\right] \delta_{n_{i}, n_{j}}\right. \\
\left.-i e_{x} \frac{a_{w}}{L_{x}} I_{n_{i} n_{j}}^{x} \delta_{m_{i}, m_{j}}-i e_{y}\left[-\sqrt{\frac{m_{j}+1}{2}} \delta_{m_{i}, m_{j}+1}+\sqrt{\frac{m_{j}}{2}} \delta_{m_{i}, m_{j}-1}\right] \delta_{n_{i}, n_{j}}\right\} .
\end{gathered}
$$

Finally, we obtain $g_{i j}$ by applying the unitary transformation

$$
g_{i j}=\left\langle\psi_{i}|g| \psi_{j}\right\rangle=\left\langle m_{i}, n_{i}\left|U^{\dagger} g U\right| m_{j}, n_{j}\right\rangle
$$

The $g_{i j}$ matrix elements can also be calculated directly from (A.24) using numerical integration. In order to do that the derivatives of $\psi_{i}(\mathbf{r})$ with respect to $x$ and $y$ need to be calculated and saved. This method is less accurate but is good for comparison. 



\section{B. Convergence of Coulomb matrix elements}

I begin by rewriting $\mathcal{I}_{j r}$ using

$$
\begin{aligned}
\psi_{j}^{*}\left(\mathbf{r}^{\prime}\right) \psi_{r}\left(\mathbf{r}^{\prime}\right) & =\left\{\psi_{j}^{*}\left(\mathbf{r}^{\prime}\right)-\psi_{j}^{*}(\mathbf{r})\right\}\left\{\psi_{r}\left(\mathbf{r}^{\prime}\right)-\psi_{r}(\mathbf{r})\right\} \\
& +\psi_{j}^{*}\left(\mathbf{r}^{\prime}\right) \psi_{r}(\mathbf{r})+\psi_{j}^{*}(\mathbf{r}) \psi_{r}\left(\mathbf{r}^{\prime}\right)-\psi_{j}^{*}(\mathbf{r}) \psi_{r}(\mathbf{r})
\end{aligned}
$$

so

$$
\begin{aligned}
\frac{4 \pi \varepsilon}{q^{2}} \mathcal{I}_{r j}(\mathbf{r})= & \int \frac{\left\{\psi_{j}^{*}\left(\mathbf{r}^{\prime}\right)-\psi_{j}^{*}(\mathbf{r})\right\}\left\{\psi_{r}\left(\mathbf{r}^{\prime}\right)-\psi_{r}(\mathbf{r})\right\}}{\left|\mathbf{r}-\mathbf{r}^{\prime}\right|+\eta} \mathrm{d} \mathbf{r}^{\prime} \\
+ & \int \frac{\psi_{j}^{*}\left(\mathbf{r}^{\prime}\right) \psi_{r}(\mathbf{r})+\psi_{j}^{*}(\mathbf{r}) \psi_{r}\left(\mathbf{r}^{\prime}\right)}{\left|\mathbf{r}-\mathbf{r}^{\prime}\right|+\eta} \mathrm{d} \mathbf{r}^{\prime} \\
& -\psi_{j}^{*}(\mathbf{r}) \psi_{r}(\mathbf{r}) \int \frac{1}{\left|\mathbf{r}^{\prime}-\mathbf{r}\right|+\eta} \mathrm{d} \mathbf{r}^{\prime}
\end{aligned}
$$

The three integrals on the r.h.s. in (B.3) I will denote as $\tilde{\mathcal{I}}_{r j}, \mathcal{I}_{r j}^{(2)}$ and $\mathcal{I}_{r j}^{(3)}$ counting from left to right. It should be noted that technically $\mathcal{I}_{r j}^{(2)}$ is infinite for $\eta=0$ and $\mathcal{I}_{r j}^{(3)}$ is infinite for all $\eta$ because the area of integration is infinite. We could take the limit of infinitely large integration area and zero $\eta$ but for clarity it will be suppressed. In the end we will see that Hamiltonian matrix elements arising from the $\mathcal{I}_{r j}^{(2)}$ and $\mathcal{I}_{r j}^{(3)}$ terms are zero and independent of $\eta$ and the size of the integration area.

Let's start with $\mathcal{I}_{r j}^{(3)}$. Define

$$
A(\eta) \equiv \int \frac{1}{\left|\mathbf{r}^{\prime}-\mathbf{r}\right|+\eta} \mathrm{d} \mathbf{r}^{\prime}
$$

Note that $A(\eta)$ is independent of $\mathbf{r}$ since the integration area is large (but not yet infinite) and $\mathbf{r}$ is simply a shift in the integration variable. The contribution of $\mathcal{I}_{r j}^{(3)}$ to the Coulomb interaction Hamiltonian $\mathcal{H}_{C}$ is

$$
\begin{aligned}
\mathcal{H}_{C}^{(3)} & =\sum_{i j r s}\left\langle i\left|\mathcal{I}_{r s}^{(3)}\right| s\right\rangle d_{i}^{\dagger} d_{j}^{\dagger} d_{s} d_{r} \\
& =-\frac{q^{2}}{4 \pi \varepsilon} A(\eta) \sum_{i j r s}\left(\int \psi_{i}^{*}(\mathbf{r}) \psi_{j}^{*}(\mathbf{r}) \psi_{s}(\mathbf{r}) \psi_{r}(\mathbf{r}) \mathrm{d} \mathbf{r}\right) d_{i}^{\dagger} d_{j}^{\dagger} d_{s} d_{r}
\end{aligned}
$$




\section{B. Convergence of Coulomb matrix elements}

Note that if we swap $d_{s}$ and $d_{r}$ in (B.6) we get a factor of -1 due to the anti-commutation relations of the fermionic operators. However, swapping $s$ and $r$ in the integral has no effect. We can therefore see that (B.6) must be zero (independent of $\eta$ and the integration domain).

It is a bit more involved to show that the contribution to the Coulomb Hamiltonian from $\mathcal{I}_{r j}^{(2)}$ is zero. Let's start by noting that

$$
\mathcal{H}_{C}=\frac{1}{2} \sum_{i j r s}\left\langle i j\left|V_{C}\right| r s\right\rangle d_{i}^{\dagger} d_{j}^{\dagger} d_{s} d_{r}=-\frac{1}{2} \sum_{i j r s}\left\langle i j\left|V_{C}\right| r s\right\rangle d_{j}^{\dagger} d_{i}^{\dagger} d_{s} d_{r}
$$

where I've used the anti-commutation relation $d_{i} d_{j}=-d_{j} d_{i}$. The $i$ and $j$ are only dummy variables being summed over so we can interchange them and get

$$
-\frac{1}{2} \sum_{i j r s}\left\langle i j\left|V_{C}\right| r s\right\rangle d_{j}^{\dagger} d_{i}^{\dagger} d_{s} d_{r}=-\frac{1}{2} \sum_{i j r s}\left\langle j i\left|V_{C}\right| r s\right\rangle d_{i}^{\dagger} d_{j}^{\dagger} d_{s} d_{r} .
$$

We can take this further by doing the same trick again with $r$ and $s$ and finally swapping $i$ and $j$ back to obtain

$$
\begin{aligned}
\sum_{i j r s}\left\langle i j\left|V_{C}\right| r s\right\rangle d_{i}^{\dagger} d_{j}^{\dagger} d_{s} d_{r} & =-\sum_{i j r s}\left\langle j i\left|V_{C}\right| r s\right\rangle d_{i}^{\dagger} d_{j}^{\dagger} d_{s} d_{r} \\
& =-\sum_{i j r s}\left\langle i j\left|V_{C}\right| s r\right\rangle d_{i}^{\dagger} d_{j}^{\dagger} d_{s} d_{r} \\
& =+\sum_{i j r s}\left\langle j i\left|V_{C}\right| s r\right\rangle d_{i}^{\dagger} d_{j}^{\dagger} d_{s} d_{r}
\end{aligned}
$$

This allows us to rewrite the Coulomb Hamiltonian as

$$
\mathcal{H}_{C}=\frac{1}{8} \sum_{i j r s}\left\{\left\langle i j\left|V_{C}\right| r s\right\rangle-\left\langle j i\left|V_{C}\right| r s\right\rangle-\left\langle i j\left|V_{C}\right| s r\right\rangle+\left\langle j i\left|V_{C}\right| s r\right\rangle\right\} d_{i}^{\dagger} d_{j}^{\dagger} d_{s} d_{r}
$$

The above is written for the total Coulomb interaction Hamiltonian $\mathcal{H}_{C}$ but it is also valid for the part of the Hamiltonian arising from the $\mathcal{I}_{r j}^{(2)}$ term. This means we can write

$$
\begin{aligned}
\mathcal{H}_{C}^{(2)} & \equiv \frac{1}{2} \sum_{i j r s}\left\langle i\left|\mathcal{I}_{r j}^{(2)}\right| s\right\rangle d_{i}^{\dagger} d_{j}^{\dagger} d_{s} d_{r} \\
& =\frac{1}{8} \sum_{i j r s}\left\{\left\langle i\left|\mathcal{I}_{j r}^{(2)}\right| s\right\rangle-\left\langle j\left|\mathcal{I}_{i r}^{(2)}\right| s\right\rangle-\left\langle i\left|\mathcal{I}_{j s}^{(2)}\right| r\right\rangle+\left\langle j\left|\mathcal{I}_{i s}^{(2)}\right| r\right\rangle\right\} d_{i}^{\dagger} d_{j}^{\dagger} d_{s} d_{r}
\end{aligned}
$$

Now all we have to do is show that the quantity in the curly brackets in eq. (B.13) is zero for all $i, j, r, s$. Let's begin by defining

$$
F_{r}(\mathbf{r}, \eta) \equiv \int \frac{\psi_{r}\left(\mathbf{r}^{\prime}\right)}{\left|\mathbf{r}^{\prime}-\mathbf{r}\right|+\eta} \mathrm{d} \mathbf{r}^{\prime}
$$


We then have

$$
\frac{4 \pi \varepsilon}{q^{2}} \mathcal{I}_{r j}^{(2)}=\psi_{r}(\mathbf{r}) F_{j}^{*}(\mathbf{r}, \eta)+\psi_{j}^{*}(\mathbf{r}) F_{r}(\mathbf{r}, \eta)
$$

so

$$
\begin{gathered}
\left\langle i\left|\mathcal{I}_{j r}^{(2)}\right| s\right\rangle-\left\langle j\left|\mathcal{I}_{i r}^{(2)}\right| s\right\rangle-\left\langle i\left|\mathcal{I}_{j s}^{(2)}\right| r\right\rangle+\left\langle j\left|\mathcal{I}_{i s}^{(2)}\right| r\right\rangle= \\
\int\left\{\psi_{i}^{*}\left[\psi_{r} F_{j}^{*}+\psi_{j}^{*} F_{r}\right] \psi_{s}-\psi_{j}^{*}\left[\psi_{r} F_{i}^{*}+\psi_{i}^{*} F_{r}\right] \psi_{s}\right. \\
\left.-\psi_{i}^{*}\left[\psi_{s} F_{j}^{*}+\psi_{j}^{*} F_{s}\right] \psi_{r}+\psi_{j}^{*}\left[\psi_{s} F_{i}^{*}+\psi_{i}^{*} F_{s}\right] \psi_{r}\right\} \mathrm{d} \mathbf{r} .
\end{gathered}
$$

Note that I have omitted the variables $\mathbf{r}$ and $\eta$ for easier reading. Looking at the 8 terms in eq. (B.16) we see that they all cancel out. We have therefore shown that $\mathcal{H}_{C}^{(2)}=0$. Combined with our previous result that $\mathcal{H}_{C}^{(3)}=0$ we can write the total Coulomb Hamiltonian as

$$
\mathcal{H}_{C}=\frac{1}{2} \sum_{i j r s}\left\langle i\left|\tilde{\mathcal{I}}_{j r}\right| s\right\rangle d_{i}^{\dagger} d_{j}^{\dagger} d_{s} d_{r}
$$





\section{Bibliography}

[1] Georg Heinrich, J. G. E. Harris, and Florian Marquardt. Photon shuttle: Landauzener-stückelberg dynamics in an optomechanical system. Phys. Rev. A, 81:011801, Jan 2010 .

[2] P. Rabl. Photon blockade effect in optomechanical systems. Phys. Rev. Lett., 107:063601, Aug 2011.

[3] C. Liu, Z. Dutton, C. H. Behroozi, and L. V. Hau. Observation of coherent optical information storage in an atomic medium using halted light pulses. Nature, 409:490-493, 2001.

[4] T. Golubeva, Yu. Golubev, O. Mishina, A. Bramati, J. Laurat, and E. Giacobino. High-speed spatially multimode atomic memory. Phys. Rev. A, 83:053810, May 2011.

[5] T. Yoshie, A. Scherer, J. Hendrickson, G. Khitrova, G. Rupper H. M. Gibbs, O. B. Shchekin C. Ell, and D. G. Deppe. Vacuum rabi splitting with a single quantum dot in a photonic crystal nanocavity. Nature, 432:200-203, 2004.

[6] E. Peter, P. Senellart, D. Martrou, A. Lemaître, J. Hours, J. M. Gérard, and J. Bloch. Exciton-photon strong-coupling regime for a single quantum dot embedded in a microcavity. Phys. Rev. Lett., 95:067401, Aug 2005.

[7] K. Hennessy, A. Badolato, M. Winger, D. Gerace, M. Atatüre, S. Gulde, S. Fält, E. L. Hu, and A. Imamoğlu. Quantum nature of a strongly coupled single quantum dot-cavity system. Nature, 445:896-899, 2007.

[8] David Press, Stephan Götzinger, Stephan Reitzenstein, Carolin Hofmann, Andreas Löffler, Martin Kamp, Alfred Forchel, and Yoshihisa Yamamoto. Photon antibunching from a single quantum-dot-microcavity system in the strong coupling regime. Phys. Rev. Lett., 98:117402, Mar 2007.

[9] A. Wallraff, D. I. Schuster, L. Frunzio A. Blais, R.-S. Huang, J. Majer, S. Kumar, S. M. Girvin, and R. J. Schoelkopf. Strong coupling of a single photon to a superconducting qubit using circuit quantum electrodynamics. Nature, 431:162-167, 2004. 


\section{BIBLIOGRAPHY}

[10] T. Niemczyk, F. Deppe, H. Huebl, E. P. Menzel, F. Hocke, M. J. Schwarz, J. J. Garcia-Ripoll, D. Zueco, T. Hümmer, E. Solano, A. Max, , and R. Gross. Circuit quantum electrodynamics in the ultrastrong-coupling regime. Nature Physics, 6:772-776, 2010.

[11] E. Hoffmann, F. Deppe, T. Niemczyk, T. Wirth, E. P. Menzel, H. Huebl G. Wild, M. Mariantoni, A. Lukashenko T. Weisl, A. P. Zhuravel, A. V. Ustinov, A. Marx, and R. Gross. A superconducting $180^{\circ}$ hybrid ring coupler for circuit quantum electrodynamics. Appl. Phys. Lett., 97(222508), 2010.

[12] M.R. Delbecq, V. Schmitt, F.D. Parmentier, N. Roch, J.J. Viennot, G. Fève, B. Huard, C. Mora, A. Cottet, and T. Kontos. Coupling a quantum dot, fermionic leads and a microwave cavity on-chip, 2011.

[13] M.R. Delbecq, V. Schmitt, F.D. Parmentier, N. Roch, J.J. Viennot, G. Fève, B. Huard, C. Mora, A. Cottet, and T. Kontos. Dipole coupling of a double quantum dot to a microwave resonator, 2011.

[14] H. C. Liu and Federico Capasso. Intersubband Transitions in Quantum Wells: Physics and Device Applications, volume 62. Academic Press, 1999.

[15] A. Gabbay, J. Reno, J. R. Wendt, A. Gin, M. C. Wanke, M. B. Sinclair, E. Shaner, and I. Brener. Interaction between metamaterial resonators and intersubband transitions in semiconductor quantum wells. Appl. Phys. Lett., 98(203103), 2011.

[16] C. Ciuti, G. Bastard, , and I. Carusotto. Quantum vacuum properties of the intersubband cavity polariton field. Phys. Rev. B, 72(115303), 2005.

[17] M. Devoret, S. Girvin, and R. Schoelkopf. Circuit-qed: How strong can the coupling between a josephson junction atom and a transmission line resonator be? Ann. Phys., 16:767 - 779, 2007.

[18] A. A. Abdumalikov, O. Astafiev, Y. Nakamura, Y. A. Pashkin, and J. Tsai. Vacuum rabi splitting due to strong coupling of a flux qubit and a coplanar-waveguide resonator. Phys. Rev. B, 78(180502), 2008.

[19] F. De Zela, E. Solano, and A. Gago. Micromaser without the rotating-wave approximation: The bloch-siegert shift and related effects. Opt. Commun., 142:106-118, 1997.

[20] A. T. Sornborger, A. N. Cleland, and M. R. Geller. Superconducting phase qubit coupled to a nanomechanical resonator: Beyond the rotating-wave approximation. Phys. Rev. A, 70(052315), 2004.

[21] E. K. Irish. Generalized rotating-wave approximation for arbitrarily large coupling. Phys. Rev. Lett., 99(173601), 2007. 
[22] Olafur Jonasson, Chi-Shung Tang, Hsi-Sheng Goan, Andrei Manolescu, and Vidar Gudmundsson. Quantum magneto-electrodynamics of electrons embedded in a photon cavity. New Journal of Physics, 14, in press (2012).

[23] A.L. Fetter and J.D. Walecka. Quantum theory of many-particle systems. Dover Books on Physics. Dover Publications, 2003.

[24] C. Cohen-Tannoudji, B. Diu, and F. Laloë. Quantum mechanics. Number v. 1 in Textbook physics. Wiley, 1977.

[25] D.K. Cheng. Field and wave electromagnetics. Addison-Wesley series in electrical engineering. Addison-Wesley, 1989.

[26] E.T. Jaynes and F.W. Cummings. Comparison of quantum and semiclassical radiation theories with application to the beam maser. Proceedings of the IEEE, 51(1):89 - 109, jan. 1963.

[27] J. M. Raimond, M. Brune, and S. Haroche. Manipulating quantum entanglement with atoms and photons in a cavity. Rev. Mod. Phys., 73:565-582, 2001.

[28] S. Haroche and J.-M. Raymond. Exploring the Quantum. Oxford University Press, 2006.

[29] B. W. Shore and P. L. Knight. The jaynes-cummings model. J. Mod. Opt., 40(7):1195-1238, 1993.

[30] I D Feranchuk, L I Komarov, and A P Ulyanenkov. Two-level system in a one-mode quantum field: numerical solution on the basis of the operator method. J. Phys. A, 29(4035), 1996.

[31] X.-H. Li, K.-L. Wang, and T. Liu. Ground state of jaynes-cummings model: Comparison of solutions with and without the rotating-wave approximation. Chin. Phy. Lett., 26(044212), 2009.

[32] Y. Zhang, G. Chen, Q. Liang L. Yu, J.-Q. Liang, , and S. Jia. Analytical ground state for the jaynes-cummings model with ultrastrong coupling. Phys. Rev. A, 83(065802), 2011.

[33] Vidar Gudmundsson, Olafur Jonasson, Chi-Shung Tang, Hsi-Sheng Goan, and Andrei Manolescu. Time-dependent transport of electrons through a photon cavity. Phys. Rev. B, 85, in press (2012).

[34] I.S. Gradstheyn and I.M. Ryzhik. Table of Integrals, Series and Products. 2007. 